Linköping Studies in Science and Technology

Dissertation, No. 1940

\title{
TAILORING VISUALIZATION APPLICATIONS FOR TASKS AND USERS
}

Alexander Bock

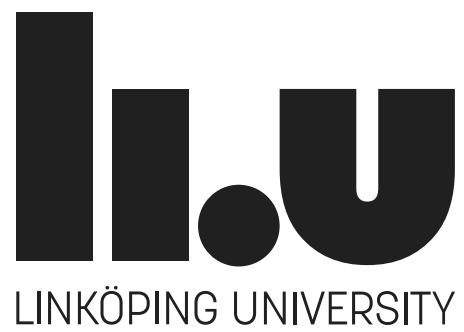

Division of Media and Information Technology

Department of Science and Technology

Linköping University, SE-601 74 Norrköping, Sweden

Norrköping, June 2018 
Tailoring Visualization Applications for Tasks and Users

Copyright () 2018 Alexander Bock (unless otherwise noted)

Division of Media and Information Technology

Department of Science and Technology

Campus Norrköping, Linköping University

SE-601 74 Norrköping, Sweden

ISBN: 978-91-7685-291-0

ISSN: 0345-7524

Printed in Sweden by LiU-Tryck, Linköping, 2018 


\section{Acknowledgments}

My first thanks goes to my two supervisors, Timo Ropinski and Anders Ynnerman, without whom this could have never happened. Timo, who could have thought that applying for a student job in 2008 would ultimately lead to this $\mathrm{PhD}$ thesis. I will be ever grateful for the help and this opportunity. Anders, thank you for revitalizing my love in everything space-related and providing me with the opportunity to work on these topics. Jag är skyldig dig en $\operatorname{drink}^{1}$ !

Thanks to all my friends I had the pleasure of meeting throughout the years. Indre, ačiu už tai, kad manes neužmušei kai kažkas galejjo paminèti istoriškai netikslų šalies pavadinimą $^{2}$; you, Johan, and Freja keep fabulous Norrköping lovely! Paula, thanks for showing me around the world and being such a good friend and moral compass; you do you! Saghi and Ehsan, بابت مهم ترين تعطيلات زندگيم از شما تشكر ميكنم Daniel, for being my partner in crime; Umut, başından sonuna kadar (ve sonrasında da) iyi bir arkadaş olduğun için teşekkür ederim ${ }^{4}$; Erik, for making sure that every paper with you as coauthor got accepted; Emil, without whom a large part of this thesis would not have been possible; Martin, for proving that it is worth to go the extra mile; Stefan, for always being the perfect person to discuss intricacies of algorithmic details with; Khoa, my photography master; Rickard and Sathish, shared pain is half the pain; Katerina, Carlo and Lucie, for too many good times to count; Andrew and Sherilyn, to Cologne!; Noeska, bedankt dat je contact met me hebt gehouden, ondanks dat ik gestop ben met MedVis ${ }^{5}$; Big thanks also go, of course, to Joakim, Marcus, Åsa, Niclas, Jimmy, Andreas, Patric, Miro, Peter, Jochen, Ali,

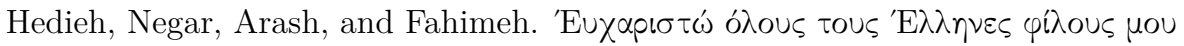

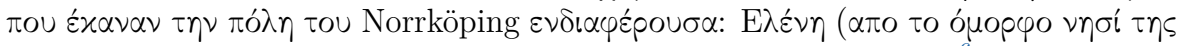

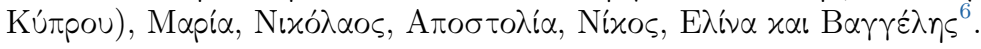

Thanks to all students that I had to pleasure of working with over the years. You rock! Sandra, Martin, Victor, HC, Jonas, Michal, Anton, Karl-Johan, Tomas, Erik, Kalle, Michael, Sebastian, Michael, Rickard, Michael, Oskar, Jonathan, Klas, Karin, Kristin, Caroline, Jonathan, Matthias, Sofie, Hannah, and Adam.

Thanks to Ingrid for helping out with many difficult questions and providing many new viewpoints on the value of applications.

To Eva Skärblom and Gun-Britt Löfgren, who helped out in so many different ways that would require their own dedication page. Tack så mycket!

\footnotetext{
${ }^{1}$ I owe you a drink.

2 for not killing me when someone might have mentioned a historically inaccurate country name

${ }^{3}$ for taking me on the most important vacation of my life

4 for being a good friend from the beginning to the end (and afterwards)

${ }^{5}$ for not breaking off contact even after turning my back on MedVis

6 Thanks to all my Greek friends for making the city interesting: Eleni (close enough), Maria, Nikolaos, Apostolia, Nikos, Elina, and Vangelis
} 
Thanks to Carter who, in many ways, is the polar opposite of me; to Masha, thank you for taking me in and for the trust throughout the years of collaboration; to Cláudio, for opening up the opportunity for a new exciting life phase.

A collective thanks to everyone at the Visualization Center $\mathrm{C}$ in Norrköping. It is a priviledge to work in a place to which other people travel for their vacation.

Vielen Dank an meine Familie für die lebenslange Unterstützung; insbesondere die zusätzlichen Schwierigkeiten die der Umzug in ein anderes Land bringt ${ }^{7}$ !

To my incredibly understanding wife Mina. Meeting and marrying someone during the second half of a $\mathrm{PhD}$ must be the worst possible timing and, yet, for some reason she did not run away from it. I do not have more to say than عاشقتم.

Norrköping, June 2018

Alexander Bock

Klas Victor Jonathan Tomas Sandra Karl-Johan Kristin Sofie Oskar Jochen Vangelis Jimmy Patric Jonas Miro Niclas Adam Arash Umut Emilia Joakim Eleni Martin Nikolaos Gun-Britt A nders Indré Khoa Rickard Andrew Åsa Anders Indre Sherilyn Saghi Mina Ehsan ${ }_{\text {Fahimeh }}$ Apostolia Joel Carter Erik Paula Timo Masha Johan Lucie Sathish Eva Marcus Leila
Negar Hedieh Noeska Cláudio Stefan Nikos Peter
Matthias Anton Karin Caroline Michal Michael Sebastian Hanna Kalle

7 Thanks also to my family for their support, especially with regards to the problems arising from moving to a different country 


\section{Abstract}

Exponential increases in available computational resources over the recent decades have fueled an information explosion in almost every scientific field. This has led to a societal change shifting from an information-poor research environment to an over-abundance of information. As many of these cases involve too much information to directly comprehend, visualization proves to be an effective tool to gain insight into these large datasets. While visualization has been used since the beginning of mankind, its importance is only increasing as the exponential information growth widens the difference between the amount of gathered data and the relatively constant human ability to ingest information. Visualization, as a methodology and tool of transforming complex data into an intuitive visual representation can leverage the combined computational resources and the human cognitive capabilities in order to mitigate this growing discrepancy.

A large portion of visualization research is, directly or indirectly, targets users in an application domain, such as medicine, biology, physics, or others. Applied research is aimed at the creation of visualization applications or systems that solve a specific problem within the domain. Combining prior research and applying it to a concrete problem enables the possibility to compare and determine the usability and usefulness of existing visualization techniques. These applications can only be effective when the domain experts are closely involved in the design process, leading to an iterative workflow that informs its form and function. These visualization solutions can be separated into three categories: Exploration, in which users perform an initial study of data, Analysis, in which an established technique is repeatedly applied to a large number of datasets, and Communication in which findings are published to a wider public audience.

This thesis presents five examples of application development in finite element modeling, medicine, urban search \& rescue, and astronomy and astrophysics. For the finite element modeling, an exploration tool for simulations of stress tensors in a human heart uses a compression method to achieve interactive frame rates. In the medical domain, an analysis system aimed at guiding surgeons during Deep Brain Stimulation interventions fuses multiple modalities in order to improve their outcome. A second analysis application is targeted at the Urban Search \& Rescue community supporting the extraction of injured victims and enabling a more sophisticated decision making strategy. For the astronomical domain, first, an exploration application enables the analysis of time-varying volumetric plasma simulations to improving these simulations and thus better predict space weather. A final system focusses on combining all three categories into a single application that enables the same tools to be used for Exploration, Analysis, and Communication, thus requiring the handling of large coordinate systems, and high-fidelity rendering of planetary surfaces and spacecraft operations. 



\section{Populärvetenskaplig Sammanfattning}

De senaste decennierna har det skett en exponentiell ökning av tillgängliga beräkningsresurser vilket har lett till en informationsexplosion inom många vetenskapliga områden. Samhället har därmed gått från att vara informationsfattigt till att ha överflöd av information. Människans förmåga att ta in information är dock relativt oföränderlig. Visualisering har här visat sig vara ett effektivt verktyg för att få bättre insikt i all information. Även om visualisering har använts sedan mänsklighetens begynnelse har dess betydelse därmed ökat markant i takt med informationstillväxten. Visualisering, som metod och verktyg, utnyttjar beräkningsresurser och den mänskliga kognitiva förmågan för att omvandla komplexa data till intuitiva visuella representationer och därmed mildra informationsöverflödet.

Mycket av forskningen inom visualisering riktar sig direkt eller indirekt till användare i en specifik domän, till exempel medicin, biologi, fysik med mera. Tillämpad forskning syftar till att skapa visualiseringsapplikationer eller system som löser ett specifikt problem inom en domän. Genom att tillämpa allmän visualiseringsforskning på ett konkret problem skapas möjligheter att jämföra och analysera användbarheten av den forskningen. Visualiseringsapplikationer kräver att domänexperter är inblandade i en designprocess, som ofta involverar ett iterativt arbetsflöde, för att ta fram deras form och funktion. Applikationerna kan kategoriseras in i tre olika områden. Utforskning, där användarna utför en första analys av data. Analys, där samma teknik används upprepade gånger på många olika dataset. Kommunikation, där resultaten visas för en bredare allmän publik.

Den här avhandlingen presenterar fem exempel på applikationsutveckling inom områdena finita elementmetodsmodellering, medicin, spanings- och räddningstjänst samt astronomi. För finita elementmetodsmodelleringen presenteras ett verktyg och komprimeringsmetod för att analysera simuleringar av stresstensorer i ett mänskligt hjärta. För den medicinska domänen presenteras ett analyssystem som hjälper kirurger att styra elektroder vid hjärnstimuleringsoperationer. Här kombineras flera olika modaliteter för att förbättra resultatet av operationen. För spaningsoch räddningstjänsten presenteras ett analyssystem som hjälper dem att hitta och rädda skadade personer genom ett ett mer sofistikerat beslutstödssystem. Inom astromidomänen presenteras först en utforskningsapplikation för tidsvarierande plasmasimuleringar som används för att förbättra rymdvädersimuleringar. Slutligen presenteras ett system som fokuserar på att kombinera alla tre kategorier i en enda applikation. Samma verktyg kan därmed användas för utforskning, analys och publik framställning. Inom astronomidomänen måste ett sådant verktyg kunna hantera koordinatsystem med stor utsträckning och högkvalitativ återgivning av planetytor samt rymduppdrag. 



\section{Publications}

The following list of publications have been included in this thesis:

Paper A: A. Bock, E. Sundén, B. Liu, B. Wuensche, and T. Ropinski. CoherencyBased Curve Compression for High-Order Finite Element Model Visualization. IEEE Transactions on Visualization and Computer Graphics, 18(12):2315-2324, 2012

Paper B: A. Bock, N. Lang, G. Evangelista, R. Lehrke, and T. Ropinski. Guiding Deep Brain Stimulation Interventions by Fusing Multimodal Uncertainty Regions. In Proceedings of the Pacific Visualization Symposium (Pacific Vis), pages 97-104. IEEE, 2013

Paper C: A. Bock, A. Kleiner, J. Lundberg, and T. Ropinski. Supporting Urban Search \& Rescue Mission Planning through Visualization-Based Analysis. In Vision, Modeling $\&$ Visualization. The Eurographics Association, 2014

Paper D: A. Bock, A. Kleiner, J. Lundberg, and T. Ropinski. An Interactive Visualization System for Urban Search \& Rescue Mission Planning. In International Symposium on Safety, Security, and Rescue Robotics. IEEE, 2014

Paper E: A. Bock, Å. Svensson, A. Kleiner, J. Lundberg, and T. Ropinski. A Visualization-Based Analysis System for Urban Search \& Rescue Mission Planning Support. Computer Graphics Forum, 36(6):148-159, 2016

Paper F: A. Bock, A. Pembroke, M. L. Mays, L. Rastaetter, A. Ynnerman, and T. Ropinski. Visual Verification of Space Weather Ensemble Simulations. In Proceedings of the Scientific Visualization Conference. IEEE, 2015

Paper G: E. Axelsson, J. Costa, C. T. Silva, C. Emmart, A. Bock, and A. Ynnerman. Dynamic Scene Graph: Enabling Scaling, Positioning, and Navigation in the Universe. Computer Graphics Forum, 36(3):459-468, 2017

Paper H: K. Bladin, E. Axelsson, E. Broberg, C. Emmart, P. Ljung, A. Bock, and A. Ynnerman. Globe Browsing: Contextualized Spatio-Temporal Planetary Surface Visualization. IEEE Transactions on Visualization and Computer Graphics, 24(1):802-811, 2017

Paper I: A. Bock, C. Emmart, M. Kuznetsova, and A. Ynnerman. OpenSpace: Changing the Narrative of Public Disseminations in Astronomical Visualization from What to How. IEEE Computer Graphics and Applications, Special Issue - Applied Vis, 38(3), 2018, to appear May/June 2018 
The following publications, reported in reverse chronological order, are related to the work described in this thesis, but have not been included:

- A. Bock, E. Axelsson, K. Bladin, J. Costa, G. Payne, M. Territo, J. Kilby, E. Myers, M. M. Kuznetsova, C. Emmart, and A. Ynnerman. OpenSpace: An Open-Source Astrovisualization Framework. Journal of Open-Source Software, 2(15):281, 2017

- A. Bock, A. Pembroke, M. L. Mays, and A. Ynnerman. OpenSpace: An OpenSource Framework for Data Visualization and Contextualization. In American Geophysical Union Fall Meeting Abstracts, pages IN42A-03, 2015

- A. Bock, M. Marcinkowski, J. Kilby, C. Emmart, and A. Ynnerman. OpenSpace: Public Dissemination of Space Mission Profiles. In Proceedings of the Scientific Visualization Conference (Poster), pages 141-142. IEEE, 2015

- M. E. Dieckmann, A. Bock, H. Ahmed, D. Doria, G. Sarri, A. Ynnerman, and M. Borghesi. Shocks in Unmagnetized Plasma with a Shear Flow: Stability and Magnetic Field Generation. Journal of Plasma Physics, 22(7):072104, 2015

- A. Bock, M. L. Mays, L. Rastaetter, A. Ynnerman, and T. Ropinski. VCMass: A Framework for Verification of Coronal Mass Ejection Ensemble Simulations. In Proceedings of the Scientific Visualization Conference (Poster). IEEE, 2014

- E. Sundén, A. Bock, D. Jönsson, A. Ynnerman, and T. Ropinski. Interaction Techniques as a Communication Channel when Presenting 3D Visualizations. In VIS International Workshop on 3DVis, pages 61-64. IEEE, 2014

- S. Lindholm, M. Falk, E. Sundén, A. Bock, A. Ynnerman, and T. Ropinski. Hybrid Data Visualization Based On Depth Complexity Histogram Analysis. Computer Graphics Forum, 34(1):74-85, 2014

- S. Lindholm and A. Bock. Poor Man's Rendering of Segmented Data. In Proceedings of SIGRAD, volume 94, pages 49-54, 2013

- K. T. Nguyen, A. Bock, A. Ynnerman, and T. Ropinski. Deriving and Visualizing Uncertainty in Kinetic PET Modeling. In Proceedings of the Workshop on Visual Computing for Biology and Medicine, pages 107-114. Eurographics, 2012

- B. Liu, A. Bock, T. Ropinski, M. Nash, P. Nielsen, and B. Wuensche. GPUAccelerated Direct Volume Rendering of Finite Element Data Sets. In Proceedings of the Conference on Image and Vision Computing New Zealand, pages 109-114. ACM, 2012 


\title{
Contributions
}

\author{
Paper A: Coherency-Based Curve Compression for High-Order Finite \\ Element Visualization \\ Presents a rendering technique for real-time visualization of non-linar finite element \\ models by introducing a preprocessing step in which potential rays are precomputed \\ by solving non-linear transformations and then compressed using B-splines. Using \\ the proxy rays during ray marching as an approximation for these transformations \\ leads to a performance improvement of $15 \times$ compared to straight-forward GPU \\ implementations. This work was presented at IEEE VisWeek 2012.
}

Paper B: Guiding Deep Brain Stimulation Interventions by Fusing Multimodal Uncertainty Regions

In a participatory design with expert brain surgeons, this work presents a system that supports Deep Brain Stimulation operations placing an electrode in the patient's subthalamic nucleus. The presented system uses the available modalities, such as preoperative $\mathrm{CT} / \mathrm{MRI}$ scans, interoperative X-ray, probe measurements, and patient responses, and fuses the available information into a multiview system that presents the available uncertainty ranges to the surgeon during the operation. This work was presented at the IEEE Pacific Visualization Symposium 2013.

Paper C: Supporting Urban Search \& Rescue Mission Planning through Visualization-Based Analysis

Presents a decision support system displaying a 3D visualization of point cloud measurements obtained from partially collapsed buildings containing potentially trapped and injured victims. The system uses these point clouds for a semiautomatic path finding algorithm which suggests paths to an operator who uses combined Scientific and Information Visualization techniques to analyse different path attributes. This paper describes the results of an online study of this system with nine international expert participants. This work was presented at the International Symposium of Vision, Modeling, and Visualization in 2014.

Paper D: An Interactive Visualization System for Urban Search \& Rescue Mission Planning

This work presents an improvement to the decision support system published in Paper $\mathrm{C}$ that focuseses on the rescue experts. Different aspects of an online user study are presented as well as implementations to enable visualization of the point cloud, paths, and derived data in immersive environments. This work was presented at the International Symposium on Safety, Security, and Rescue Robotics in 2014.

Paper E: A Visualization-Based Analysis System for Urban Search \& Rescue Mission Planning Support

Based on the findings of Papers $\mathrm{C}$ and $\mathrm{D}$, this work includes an adaptive sampling method that replaces the previous brute force sampling of the path search space for 
improved efficiency. Additional visualization techniques such as projective texturing and bump mapping are included to convey additional information to the rescuer. Lastly, the work contains an additional eye-tracking user study with four rescuers. This work was published in Computer Graphics Forum in 2016.

Paper F: Visual Verification of Space Weather Ensemble Simulations Presents a visualization system developed in collaboration with space weather analysts for the use in the investigation of space weather. The system enables the comparison of in-situ measurements performed by satellites with time-varying volumetric simulations of the solar system. The system was designed in participatory design with the experts at the Community Coordinated Modeling Center, located at NASA's Goddard Space Flight Center and enabled new discoveries about the structure of coronal mass ejections. This work was presented at IEEE Vis in 2016.

Paper G: Dynamic Scene Graph: Enabling Scaling, Positioning, and Navigation in the Universe

By utilizing a dynamic coordinate system origin, the framework described in this work supports the simultaneous rendering of scenes with an extent that is larger than the precision of floating points would otherwise allow. The paper analyses the precision loss that occurs due to floating point arithmetic and, based on these findings, presents a solution that operates on dynamically traversing a scene graph structure. This work was presented at EuroVis in 2017.

Paper H: Globe Browsing: Contextualized Spatio-Temporal Planetary Surface Visualization

This paper presents a system that uses a chunked, level-of-detail rendering techniques for the high-fidelity rendering of planetary surfaces, including static and time-varying imagery data and digital elevation models of Earth, the Moon, Mars, and Pluto. Using these techniques, it becomes possible to make an extensive library of scientific surface data available to the public in their correct spatial context. This work was presented at IEEE Vis in 2017.

Paper I: OpenSpace: Changing the Narrative of Public Disseminations in Astronomical Visualization from What to How

This work presents the open-source framework OpenSpace which supports the interactive visualization of astronomical data in traditional and immersive environments. The paper advocates the use of shared, immersive experiences as an efficient medium of science dissemination to the general public and provides an overview of the required techniques to achieve this. The examples presented in the work include various spacecraft missions, such as New Horizons, Rosetta, and OSIRIS-REx, as well as planetary rendering as described in Paper $\mathrm{H}$, and the space weather visualization as described in Paper F. This work is accepted for publication in Computer Graphics \& Applications 2018. 


\section{Contents}

Acknowledgments iii

Abstract $\quad$ V

Populärvetenskaplig Sammanfattning vii

$\begin{array}{ll}\text { List of publications } & \text { ix }\end{array}$

Contributions $\quad x i$

1 Motivation 1

2 Introduction $\quad 5$

2.1 Visualization 5

2.1.1 Benefits of Visualization 6

$\begin{array}{lll}\text { 2.1.2 Limitations and Dangers of Visualization } & 7\end{array}$

2.2 The Visualization Pipeline 9

2.2.1 Data Acquisition 11

2.2.2 Direct Volume Rendering 14

2.3 The Human-in-the-Loop Model 15

2.4 Visualization Applications 16

2.4.1 Forms of Collaborative Research 16

2.4.2 Fundamentals of Application Design 17

2.4.3 Classification of Visualization Tasks 18

$\begin{array}{ll}\text { 2.4.4 Visualization Application Categories } & 19\end{array}$

3 Visualization Application Design (contributions) 23

3.1 Finite Element Models 24

3.1.1 Domain and Scientific Problems 24

3.1.2 Application Requirements 25

$\begin{array}{lll}3.1 .3 & \text { Algorithm } & 25\end{array}$

3.2 Deep Brain Stimulation Interventions 30

3.2.1 Domain and Scientific Problems 31

3.2.2 Application Requirements 32

3.2.3 Contextual View 32

3.2.4 Audio visualization 34

3.2.5 Target closeup $\quad 35$

3.2.6 System 35

3.2.7 Evaluation 36

3.3 Urban Search \& Rescue 37 
3.3.1 Domain and Scientific Problems 37

3.3.2 Application Requirements 38

$\begin{array}{lll}3.3 .3 & \text { Voxel Binning } & 38\end{array}$

3.3.4 Path Computation 41

3.3.5 Rendering 42

3.3.6 System $\quad 45$

$\begin{array}{lll}3.3 .7 & \text { Evaluation } & 47\end{array}$

3.4 Space Weather Visualization 47

3.4.1 Domain and Scientific Problems 47

3.4.2 Application Requirements 49

3.4.3 Ensemble Glyph Mapping $\quad 50$

3.4.4 Optical Flow Analysis $\quad 51$

3.4.5 Rendering $\quad 52$

3.4.6 System $\quad 54$

$\begin{array}{lll}3.5 & \text { Astronomical Visualization } & 56\end{array}$

$\begin{array}{lll}3.5 .1 & \text { OpenSpace } & 56\end{array}$

3.5.2 Dynamic Scene Graph $\quad 57$

3.5.3 Planetary Rendering 60

3.5.4 Spacecraft Missions and Dissemination 65

4 Reflections $\quad 73$

$\begin{array}{ll}\text { Bibliography } & 77\end{array}$

$\begin{array}{ll}\text { Publications } & 91\end{array}$

Paper A: Coherency-based curve compression for high-order finite element model visualization $\quad 91$

Paper B: Guiding deep brain stimulation interventions by fusing multimodal uncertainty regions $\quad 105$

Paper C: Supporting urban search \& rescue mission planning through $\begin{array}{ll}\text { visualization-based analysis } & 117\end{array}$

Paper D: An interactive visualization system for urban search \& rescue mission planning

Paper E: A visualization-based analysis system for urban search \& rescue mission planning support 139

Paper F: Visual verification of space weather ensemble simulations 155

Paper G: Dynamic Scene Graph: Enabling scaling, positioning, and navigation in the universe

Paper H: Globe Browsing: Contextualized spatio-temporal planetary surface visualization

Paper I: OpenSpace: Changing the narrative of public disseminations in astronomical visualization from What to How 


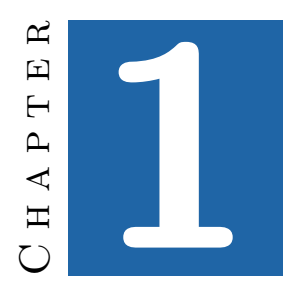

\section{Motivation}

Communicating knowledge and intentions through persistent visual means is maybe one of the most distinct features that separates humans from other animals. Our ability to intuitively understand abstract representations created by other humans from distant places or times has shaped the world's history in unimaginable ways. The fundamental reason for creating these representations, conveying information to other humans, has not changed between the earliest cave paintings 40000 years ago (see Figure 1.1(a)) and modern visualizations (see Figure 1.1(b)). While the direction of causality can be debated, humanity's focus on the use of visual representations to share knowledge and being exceptionally good at interpreting

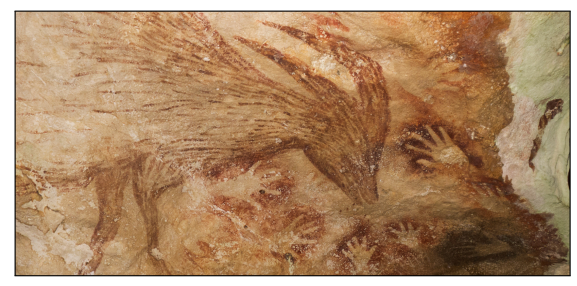

(a) The earliest known human cave painting from around 38000 BCE. Image copyright by Maxime Aubert.

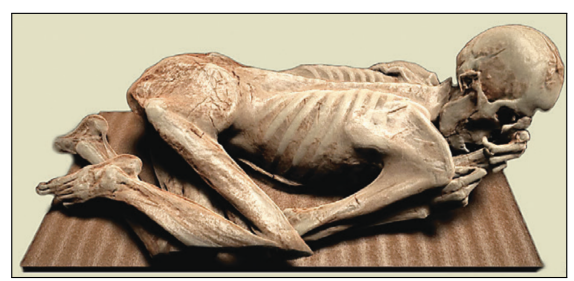

(b) A visualization using modern techniques of a pre-dynastic Egyptian mummy. Image copyright by Daniel Jönsson.

Figure 1.1: Two examples of visualizations created by humanity that are 40000 years apart. While the technological methods for their creation changed drastically, the ultimate purpose to convey information to other humans remains the same. 


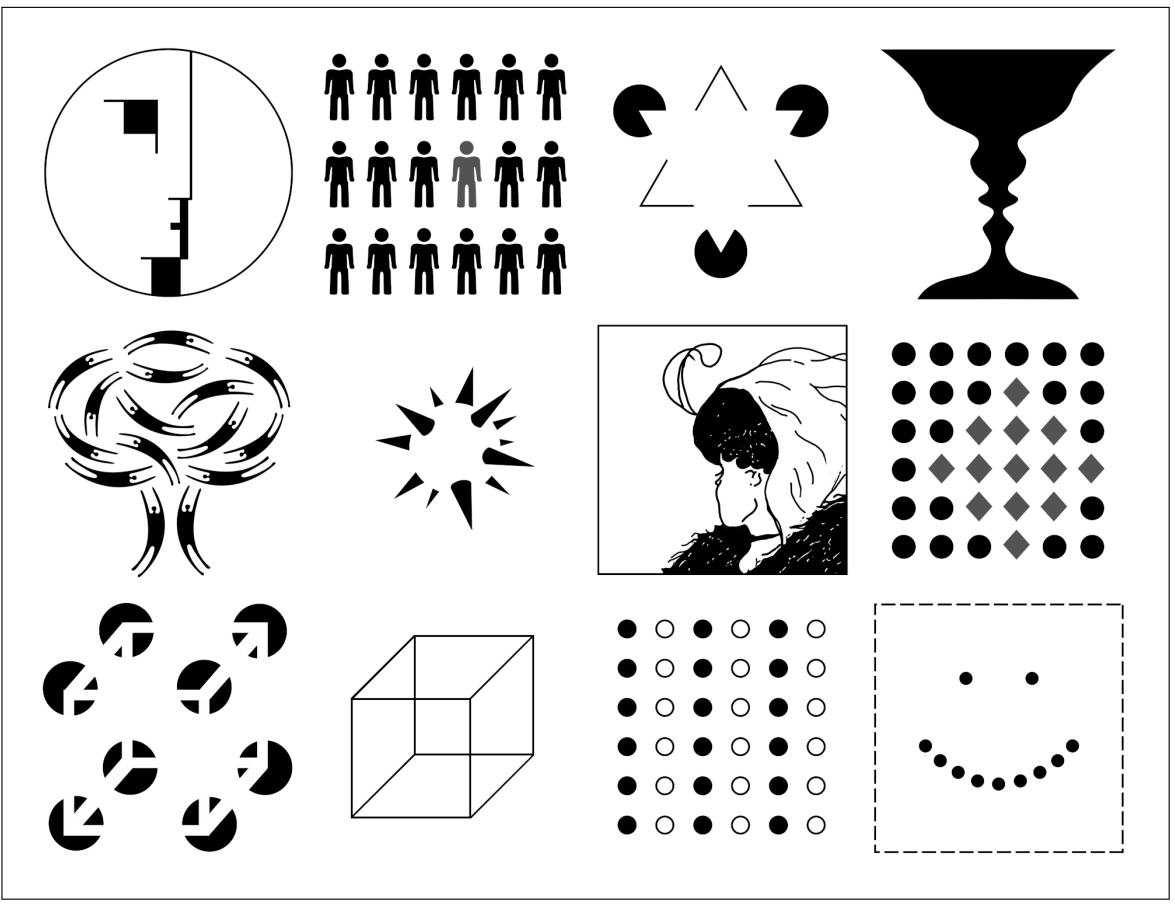

Figure 1.2: Examples of applied Gestalt theory principles that, among others, show Continuance, Closure, Similarity, and Figure \& Ground. All individual examples are organized by Grouping.

visual language by dedicating a large portion of our brain to this task is certainly connected. It is partially for this reason why many cultures have spent so much effort and time on perfecting visual languages and metaphors. On the other hand, an image can never contain the full information and, as such, a good visualization is like a story; the designer provides all the necessary components, but the final assembly occurs in mind of the beholder and is, thus, ultimately subjective.

Good visualizations make subconscious use of some remarkable aspects of human perception. We are capable of analyzing scenes both preattentively as well as attentively. Preattentive perception happens when features of an image pop out, or are obvious to the observer without conscious effort and this effect is largely independent of the number of objects that are involved. Figure 1.2 shows an example of this effect using the Gestalt theory [127]. In his work, Wertheimer found that attributes such as closure, similarity, or continuation enable an observer to perceive a collection of objects as a continuous form (or Gestalt). He also investigated how grouped objects can be modified before the continuous form is 
destroyed. Understanding these fundamental truths about human perception is invaluable in order to create meaning full visualizations; 40000 years ago or today.

An early example of the successful use of visualization for the public good is a spatial map of the cholera outbreaks around Broad street in London in 1854 (see Figure 1.3). The prevalant theory at the time for transmission of diseases was miasmatic, or caused by bad air, and not germ theory. Many decades before Wertheimer's publication of the Gestalt theory, John Snow already made use of its concepts to gain insights about the spatial distribution of these cholera outbreaks. He marked all cholera cases on a street map and used this visualization to pinpoint the origin of the outbreak - an infected pump [109]. While this visualization

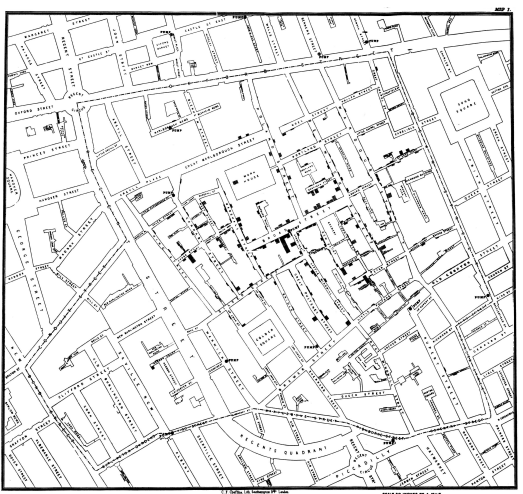

Figure 1.3: A visualization of the spatial distribution of cholera outbreaks around Broad Street in London in 1854. seems simple by today's standards, it was an important step towards the establishment of the germ theory of diseases. This is an example of a knowledge-driven approach, where a visualization is used to generate and test a hypothesis and gain an understanding of the available data.

Only a few years later, another example was created by Charles Joseph Minard in 1861 to visualize Napoleon's Russia campaign and the following retreat from Moscow in 1812 (Figure 1.4). The map visualizes six variables: geography, time, temperature, the movement of Napoleon's army, and the remaining number of troops. The layout, design, Figure 1.4: A multivariate visualization of and its ability to easily show Napoleon's Napoleon's campaign to Russian and retreat fate in Russia has caused this graphic to be called "the best statistical graphic

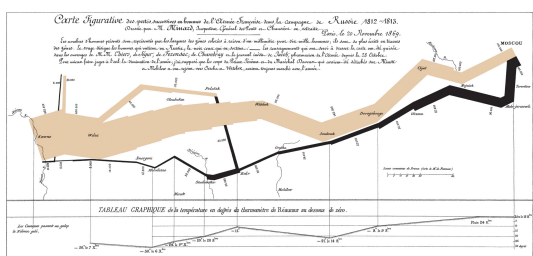

from Moscow in 1812-1813. ever drawn" [117]. As this data was produced from previously published work and mainly aimed at the general public, it serves as an example as to how visualization can be used to explain highly complex data to a public that possibly only has cursory knowledge about the topic. Besides these two examples, Tufte, in his books, provides many more good examples of visualization mixing design and information representation [116]. All of these examples show an important difference in purpose between visualization and computer graphics, which is also exemplified in Ben 


\section{Chapter $1 \cdot$ Motivation}

Shneiderman's quote that "the purpose of visualization is insight, not pictures" [25]. Instead of generating beautiful images devoid of information, the purpose of visualization is to create images that enable some person to derive insight from data. While the concept of insight is easily understood colloquially, a formal definition of it has sofar been elusive [87].

The two examples provided above allude to the fact that visualization cannot realistically occur without considering a specific target domain or problem domain. A large portion of visualization research has to take the problem domain into account and is meaningless without this context. Even for fundamental research, its effectiveness with regards to humans is important and has to be shown. Different domains are addressed through visualization applications that are tailored to an individual domain expert or a group experts, who are interested in understanding a particular aspect of their data. These experts can make use of visualization for either hypothesis generation, validation, or the communication of their theories.

Thus, the field of visualization is inherently an multidisciplinary [33], as it combines computer science, perception, cognition, interaction design and art, but also interdisciplinary it its application to other scientific fields [50]. 


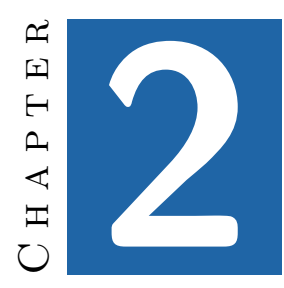

\section{Introduction}

This chapter first provides a general overview of the field of visualization with its benefits, drawbacks, and dangers in order to provide context for the rest of this thesis. Second, a commonly used variant of the visualization pipeline is introduced including some of its attributes and modifications. The third section presents a short overview of interaction design, requirements regarding the design of systems where the human-in-the-loop approach is essential, and provides information about previously published task taxonomies. The last section provides an overview of visualization systems that includes a classification system that is based on the target audience and usage for which a system is designed.

This chapter is not exhaustive and the inclined reader searching for more detailed information is referred to a number of books for an in-depth overview of the field. A general overview of the design of visualization applications is provided by Munzner in her book on "Visualization Analysis and Design" [85]. Specifically for medical applications, Preim and Botha [92] provide a detailed outline of the use of visual analysis in medicine in their book "Visual Computing for Medicine: Theory, Algorithms, and Applications".

\section{$2.1 \quad$ Visualization}

Throughout the years, there have been many attempts at finding a universally accepted definition of the scientific, design, or engineering discipline Visualization. Card et al. suggested that Visualization is "the use of computer-supported, interactive, visual representations of data to amplify cognition" [25]. Other definitions, however, place their focus on the interplay between generated images and the 
process of subjective visualization in each person [121], or generalize the concept of visualization to non-visual phenomena as well [96]. While these definitions vary widely, they overlap by focussing on the human observer and the fact that a visualization is created for and by a human, a process that inherently requires understanding of human physiology and decision making. Furthermore, the field of visualization was started as, and continues to be, a reaction to the data explosion occurring in other scientific disciplines [67], as a means to make sense of the vast quantities of data that these fields regularly generate and that generally exceed the human capacity for understanding.

The field of visualization is often separated into at least three categories; Scientific Visualization, Information Visualization, and Visual Analytics. Scientific Visualization is characterized by the use of data sources with an inherent physical and spatial component. Data traditionally attributed to Scientific Visualization comes in the form of, for example, simulations or datasets in which the spatial relationship is trivially given. Information Visualization usually deals with abstract data that does not need to possess an innate spatial component. Techniques from this part are typically high-dimensional and multi-variate. Visual Analytics places heavier focus on the analytical reasoning and the interaction modes in order to produce insight into the data rather than the source of the data itself [128].

Tory et al. [115] pointed out that these definitions require the use of words such as "usually", "typically", or "traditionally" hints at a problem with this classification scheme. For once, it is not always possible to delineate differences between the categories even in the most trivial applications of visualization [93, 126]. More complicated applications almost always use techniques from two or all three categories, increasing the difficulty of a clean classification. Additionally, from an application domain's point of view, the distinction between different categories might not even be noticeable or relevant. In their work, Tory et al. provide a more nuanced model-based taxonomy that is focusses on the characteristics of the model of the data rather than the data itself. Instead of using a taxonomy that is based on the description of the data, they propose a taxonomy that is based on the way the data is used in the visualization system and differentiates between continuous and discrete data, regardless of whether the data itself is spatial or abstract. In this thesis, no distinction is made between these visualization categories as they are providing different tools to solve the same class of problems, that is, displaying data to a human in order to facilitate insight, and "at this level there is much more they share than what separates them" [121].

\subsubsection{Benefits of Visualization}

As mentioned in the previous chapter, humans are exceptionally well adapted to interpret information contained in images. This is exemplified by the popular quote that "a picture is worth a thousand words", meaning that, for humans, 
the bandwidth to ingest information visually is much higher than through other representations. However, the computational complexity of problem classes might differ between the human visual system and computational operations. This leads to the crystallization into two classes of problems. On the one hand, there are problems that can be solved more efficiently by computers, such as searching large databases, sorting, and algorithms that typically operate on a map-and-reduce scheme. On the other hand, there are problems solved better by humans, such as pattern recognition, hypothesis forming, and others. An example of this is detecting proximity among a group of objects, which for the human perception method is of constant complexity $(\mathcal{O}(1))$, and for an algorithm at best linear $(\mathcal{O}(n))$. Visualization, being placed on the boundary between these two problem classes, can utilize the respective strengths of both computers and humans through a close integration in order to solve a larger problem set efficiently.

\subsubsection{Limitations and Dangers of Visualization}

One of the important limiting factors influencing each visualization is its subjectiveness. According to van Wijk, the benefit of using a visualization depends on "the specification [...], the perceptual skills of the observer, and the a priori knowledge of the observer" [119]. This realization is another reason why close collaboration between the visualization designer and the domain expert is of fundamental importance, as the design process has to take the experts a priori knowledge into account. Lorensen elaborated on the potential problems for the visualization community that could arise if this collaboration does not occur and summarized it as "[Visualization] has lost its customers" [67]. The fact that visualization is still alive over a decade later indicates that it indeed was possible for visualization to maintain this close collaboration. Another direct consequence of the subjectiveness is that the reproducibility of a visualization is limited to comparable consumers. A visualization system that is designed for experts in a specific field loses much of its applicability when applied to the same data models from a different field.

Another aspect of the a priori knowledge that is often overlooked is a dependence on cultural background. Whereas knowledge-based prior information can be assessed empirically, it is much harder to assess cultural biases. Some of these cultural differences can be benign, such as the Western tendency to associate movement across a red-green color scale with an increasing value, whereas East Asian cultures would associate this with a decreasing value, due to the opposite association between the red and green colors. Other differences can be seen in Figure 2.1, which displays characters from eight cartoon series built from Lego blocks and can be seen as a primitive form of visualization. Viewed in a culture that is unfamiliar with these cartoon series, however, it becomes easy to see that this visualization will be unable to produce any meaningful results to that group of users. 


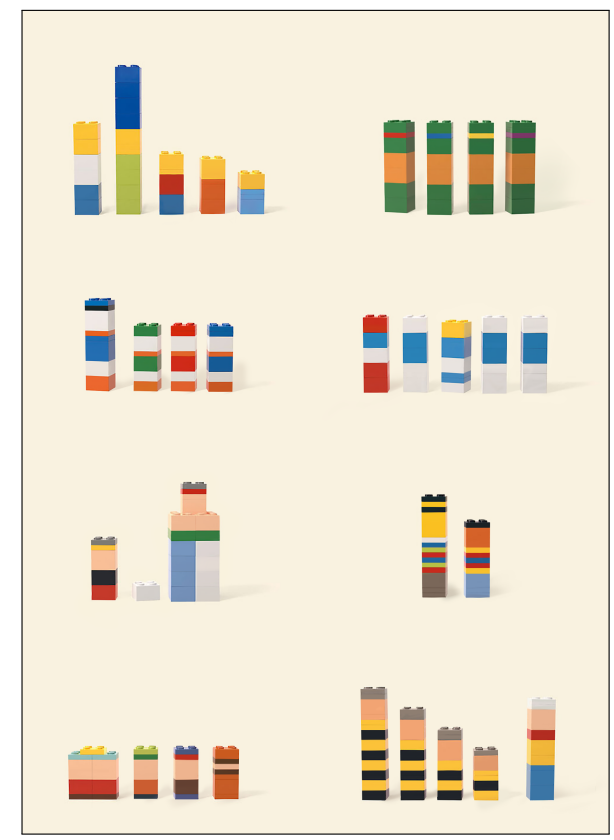

Figure 2.1: A collection of advertisement images representing (in the West) popular cartoon characters. Without the required cultural background, however, deciphering these visualizations is impossible. Image copyright by Lego.

Beside the immense benefits that visualization can provide for supporting data interpretation and hypothesis testing, the misuse of visualization can have a detrimental effect and pose a danger to the acquisition of insight. One obvious aspect outside the scope of this thesis is the use of visualization to deliberately mislead the audience. Even without a deliberate attempt, there are many pitfalls that need to be considered when designing visualizations. Verifying truths, rather than inspiring hypotheses can easily lead to confirmation biases that might lead experts to draw faulty conclusions, exemplified in the quote from van Wijk saying that "visualization should not be used to verify the final truth, but rather to inspire to new hypotheses, to be checked afterwards". Naturally, this danger is most prevalent in the initial exploration stages of a visualization and can be mitigated when a visualization system is matured and applied to many of the same types of datasets; nevertheless, it is an important aspect to consider during the design process. The remaining dangers fall into one of two categories, showing incorrect information and showing information incorrectly. The first category can occur if visualization designers apply faulty assumptions about the data by, for example, applying smoothing to inherently discrete datasets, not handling outliers correctly 


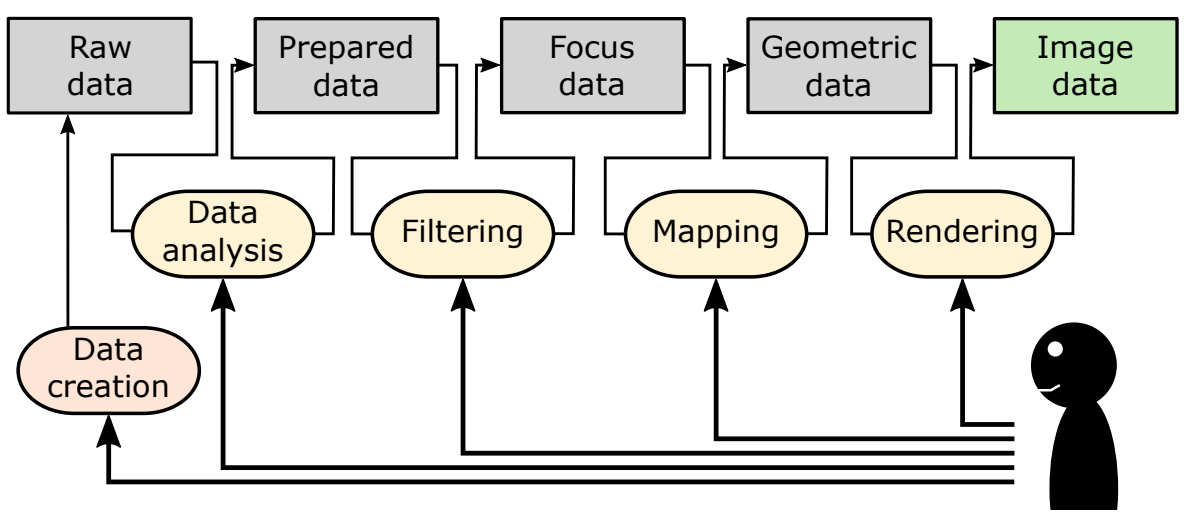

Figure 2.2: One version of the visualization pipeline as described by Dos Santos and Brodlie [35]. The acquired data is repeatedly transformed until an image is generated that can be used by the user to gain insight. The user's ability to control each part of the visualization pipeline is at the heart of the human-in-the-loop methodology.

in a filtering operation, or not considering missing data in real world datasets. For the domain expert it then becomes difficult to differentiate missing data from false data, thus eroding their trust in the visualization system. In the second category, color maps play a huge role. Ill-suited color maps trivially enable the possibility to create, highlight, or hide structures in the data without informing the expert about the process. One example is the continued use of the rainbow color map in science publications even though it has been shown to be inferior to other color maps [21].

\subsection{The Visualization Pipeline}

Figure 2.2 shows a schematic overview of the visualization pipeline. All fundamental visualization research aims at improving or impacting one or more stages in the visualization pipeline and all applied visualization research and systems utilize the concept of this pipeline. The pipeline used was first described by Haber and McNabb in 1990 [43] and later extended by Dos Santos and Brodlie in 2004 [35]. It consists of four transformations that are successively applied to the incoming data. For a complete description of the visualization pipeline and its variations, we refer to the two original works or by a survey about the development of the visualization pipeline by Moreland [81].

The input to the pipeline is the Raw data that is acquired from measurements or simulations. This data can be structure or unstructured, static, or time-varying. It is processed by the initial Data analysis, which consists of, for example, resampling, interpolation, or removal of outliers. In the Filtering step, the data is reduced with respect to the requirements of the specific task that is to be solved by, for 


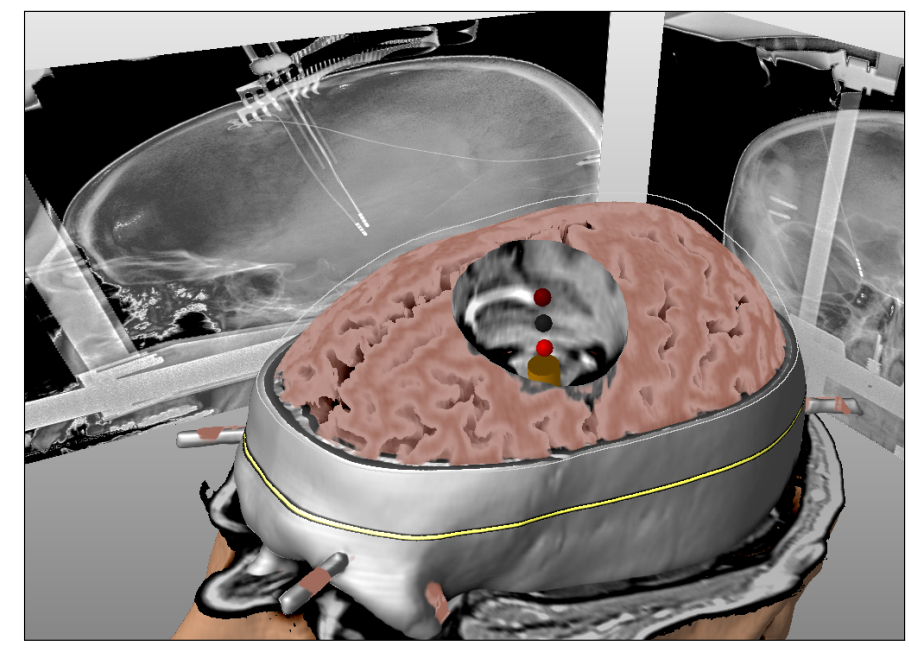

Figure 2.3: One example of a multiview visualization technique, a Magic Mirror, where X-ray scans of a patients are shown projected to the sides of a combined volumetric rendering, thus providing access to multiple modalities simultaneously.

example, thresholding, level-of-detail selection, or segmentation. This Focus data is then converted in the Mapping stage into what Haber and McNabb referred to as Abstract Visualization Objects; an abstract object containing visualizationrelated attributes, such as color, geometry, or texture, that depend on, but do not necessarily correspond to, the input data. The final step, Rendering uses these abstract representations and generates a final image that is consumed by the user. One important aspect for the design of visualization applications that was not fully accounted for in the original visualization pipeline is a feedback loop into the various transformation stages that is controlled by the user. While it has been possible to change the parameters of the Rendering or Mapping stages by, for example, changing the camera position, or changing the color attributes of the Abstract Visualization Objects of Geometric Data, for a long time, the focus of interactivity for the other steps of the pipeline has been introduced later. One of the last feedback loops, Computational Steering, described by Mulder et al. [83], enables the visualization user to directly influence the gathering or generation of the Raw Data and inspect the results with minimal delay. Closing this loop leads to the biggest gain in insight as the user can, in the example of simulations, directly understand the influence of parameter changes and can thus gain a deeper understanding of the origin of the data.

An important aspect of the design of visualization systems that is hidden from the pipeline depicted in the figure is the possibility of pipeline branching. Multiview visualization systems provide multiple simultaneous views on complementary 
aspects of the data. One example of these techniques is a magic mirror [52] as shown in Figure 2.3 that presents different aspects of the underlying data projected to the sides of a surrounding cube, thus providing the expert with additional simultaneous information about the data. In a multiview system, separate branches of the pipeline handle these views that are ultimately merged in the Rendering step. There exists a large amount of research on techniques dealing with multiview systems, an example of which is brushing or linking [114].

\subsubsection{Data Acquisition}

Regardless of its exact composition, the visualization pipeline begins with data that is either collected and measured from the real world or generated by simulations. While in the abstract, the specific form and shape of the data does not influence the visualization pipeline, concrete systems require knowledge about the origin and characteristics of specific data sources. This section elaborates on some of the attributes that are important for the contributions included in this thesis.

\section{Data structures}

There is a variety of methods to structure the acquired data. This section introduces a subset of data layouts with a focus on representations that are used in the works included in this thesis. It is by no means a complete reference as each specific problem domain can demand its own optimal data representation.

Point Cloud. Sparse point cloud data is the least structured of these data types and consists of, potentially multidimensional, measurements in a 2D or 3D space that in general do not possess any information about their connectivity. Lidar scanners are a prime example of a modality that generates unstructured point cloud. Point clouds, due to their unstructured storage, are difficult to handle and thus pose unique visualization challenges, such as handling transparency, occlusion, and the need for efficient point-based rendering techniques.

Cartesian. Multidimensional Cartesian grids are the most widely used form of structured data, 3D volumetric grids being the most applicable to this thesis. The uniform structured grid makes it possible to efficiently handle a large amount of data. A great number of rendering techniques for this type of data exist, for example isosurface rendering or direct volume rendering. The ubiquitous nature of Cartesian volumetric grids, however, also provides a major drawback. As the de facto standard data format in Scientific Visualization, it is often used in problem domains that do not produce space-filling data and where an adaptive resolution is more appropriate, thus resulting in suboptimal storage and access methods where an adaptive grid or a different underlying geometry would be better suited. 
Spherical. One non-Cartesian space-filling grid that is used in this thesis is based on spherical coordinates. Simulations of the inner solar system, for example, produce higher resolution data close to the Sun and automatically possess a spherical symmetry that can be utilized to optimize storage capacity and data access. In these cases, a spherical dataset is a 3D volume in which each of the three spherical coordinate axis, $r, \phi$, and $\theta$ is mapped to a Cartesian axis. When applying direct volume rendering to these datasets interesting characteristics, such as automatic adaptive sampling or spherical linear interpolation schemes, can be observed [4].

\section{Dimensionality}

Unfortunately, the word dimensionality is overloaded many times in Visualization. For this section, dimensionality refers to the number of values stored at each location in the dataset, rather than the number of dimensions of the dataset itself. For the purposes of this thesis a taxonomy, for example as provided by Shneiderman [108], is used that describes data as scalar, vector, tensor, or multidimensional. The difference between a 3D and a vector dataset or a tensor and a multidimensional dataset is that a vector or tensor has additional inherent information that can, and ought to, be used to restrict the creation of Abstract Visualization Objects.

Each of above categories can also be time-varying. While there have been many techniques that efficiently deal with time-varying datasets, for example time-space partitioning trees [107], efficient handling of these datasets has not been the focus of this thesis. As such, this work handles the temporal dimension analogous to the already existing spatial dimensions and, thus, considers a time-varying dataset as an ordered series of single time step datasets.

\section{Data Sources}

There are countless potential sources of datasets and a conclusive enumeration would exceed the scope of any single work. Instead, this section presents a brief overview of the different data modalities and their data acquisition techniques that are being utilized in the following contributions section.

X-ray. X-ray radiation was discovered by Wilhelm Röntgen in 1896 [97] and was quickly developed into an imaging technique. A source emits radiation that passes through the object of study. The constituent materials' absorption coefficients determine the remaining amount of radiation after passing through the object. A photosensitive plate on the other side of the object captures the remaining intensity and can thus reconstruct a representation of the object's radiotransparency, which is influenced by its density and material composition. The limitation with this technique is its restriction to a single 2D projected image of the object in question and can thus not be easily used for a 3D reconstruction. 
Computed Tomography. A Computed Tomography (CT) scanner works similar to an X-ray detector, in which the source and an electronic detector are corotating around the imaged object. Throughout this motion, many images are taken by the scanner, which are then used to reconstruct a single 3D representation of the object of study. The Nobel Prize for Physiology or Medicine was awarded to Hounsfield and Cormack in 1979 for their development of this machine [47]. The spatial and temporal resolution of the scanner and technique has since been improved by multiple orders of magnitude, enabling current machines to perform full-body scans of patients in only a few seconds or provide the ability to scan a smaller area of interest multiple times per second, thus extending the available information from the structural aspect into the functional domain. In medical applications the X-ray attenuation is measured in Hounsfield Units that measure the attenuation factor of materials and thus provides a standardized scale. As the $\mathrm{X}$-ray attenuation between different soft tissues is not very high, it is most widely used to study the skeletal structure in humans.

Magnetic Resonance Imaging. Magnetic Resonance Imaging (MRI) scanners operate by rapidly manipulating magnetic fields to force an alignment of the spins of hydrogen atoms and measuring the time for atoms to fall back to their ground state. This emits an electro-magnetic wave in the radio frequency that is detected by the scanner and used to reconstruct a 3D volumetric representation of the object of study. Since the signal is based off the availability of hydrogen atoms, MRI scans exhibit the highest resolution in areas with a high water content, such as soft tissue in human patients, whereas the skeletal structure is not well captured [30]. Comparing these attributes to a CT scanner shows that combining CT/MRI scanners provides a high resolution scanner result for a large part of the human body, thus the combination of these two modalities is often used in clinical practice.

Lidar. A Lidar scanner is another active scanning device that uses light to a similar effect as radar uses radio waves. It operates by emitting coherent light and measuring the time until the reflected light returns to the detector, thus making it possible to create a 3D line-of-sight representation of the area surrounding the scanner. These measurements can be used to create a high-resolution 3D model of, for example, humans or building structures. Combining a Lidar scanner with other scanning modalities, it becomes possible to not only detect the presence or absence of an obstacle, but also measure other physical attributes, for example surface temperature by measuring radiation emitted by an object or radial velocity through doppler shift. One important use case for Lidar scanners are autonomous vehicles that can use this information to generate an accurate, real-time 3D local environment that can be used for navigation. 


\section{Chapter $2 \bullet$ Introduction}

Simulations. The previous modalities generate data by measuring physical quantities and thus create a virtual representation of a physical phenomenon which can then be visualized. Simulations, on the other hand, utilize a minimal set of physical preconditions and try to accurately recreate the physical world and thus enable insight into areas that would either be infeasible or impossible to investigate directly. This enables the recreation of phenomenæthat cover all possible scales, but are especially useful in which direct measurements are challenging. An important distinction between the measured modalities and simulations arises in the form of noise present the data. Whereas simulations have the potential for a very low signal-to-noise ratio, any measured modality will always have some form of noise attached to the signal that has to be considered in the visual representation.

\subsubsection{Direct Volume Rendering}

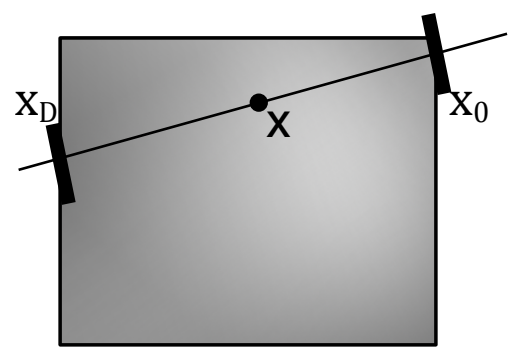

Figure 2.4: Illustration of the rendering integral for a ray with entry point $\mathbf{x}_{\mathbf{0}}$, exit point $\mathbf{x}_{\mathbf{D}}$ and an exemplary sampling point $\mathbf{x}$

ure 2.4, this results in:

$$
I\left(\mathbf{x}_{\mathbf{c}}\right)=\underbrace{I_{0}\left(\mathbf{x}_{\mathbf{0}}\right) T\left(\mathbf{x}_{\mathbf{0}}, \mathbf{x}_{\mathbf{D}}\right)}_{\text {Background }}+\int_{\mathbf{x}_{\mathbf{0}}}^{\mathbf{x}_{\mathbf{D}}} \underbrace{\sigma_{\alpha}(\mathbf{x}) I_{c}(\mathbf{x})}_{\text {Contribution }} \underbrace{T\left(\mathbf{x}, \mathbf{x}_{\mathbf{D}}\right)}_{\text {Attenuation }} \mathrm{d} \mathbf{x},
$$

where $I_{0}$ determines the background illumination, $\sigma_{\alpha}$ determines whether a sample $\mathbf{x}$ is emitting or absorbing light and $I_{e}$ specifies the amount of light contributed at a location $\mathbf{x}$, attenuated by the attenuation factor $T$, given by:

$$
T(\mathbf{a}, \mathbf{b})=\exp \left(-\int_{\mathbf{a}}^{\mathbf{b}} \tau(\mathbf{x}) \mathrm{d} \mathbf{x}\right),
$$

with $\tau(\mathbf{x})$ being the extinction coefficient that defines the occlusion of light inside the volume. Equations 2.1 and 2.2 combined are known as the volume rendering 
integral. For real-world datasets, solving the volume rendering integral analytically is not feasible and is, in practical calculations, approximated as a Riemann sum with a step size $h$ between individual samples. $h$ is a constant value whose value should be influenced by Nyquist's theorem [106].

Many volume rendering techniques can be expressed through modification of Equations 2.1 and 2.2 or their finite integration step equivalent. An example is adaptive sampling methods in which the step size $h$ depends on the encountered data values, thus being able to provide a higher sampling resolution in different parts of the volume [31]. A specialization of this is empty space skipping, where empty parts of the volume are skipped entirely in order to improve the algorithm's performance [130]. The volume rendering integral is evaluated for each pixel in the rendering window using the volume's bounding geometry. By rendering the coordinates of the volume's bounding geometry and storing the results, it is possible to generate each pixel's ray and traverse it in the graphics processing unit (GPU) [54], which has become the de facto standard in DVR.

\subsection{The Human-in-the-Loop Model}

The integration of human perception, cognition, and decision making into the knowledge discovery and analysis process is a vital aspect of any visualization system. As described by Ward et al.: "If the goal of visualization is to accurately convey information with pictures, it is essential that perceptual abilities by considered" [124]. For human perception and cognition, examples adhering to the Gestalt theory (as mentioned in Chapter 1) demonstrate the vast abilities of the human visual system in recognizing clusters, independent from the number of items, based only on simple features such as color, orientation, grouping, or closure. When designing visualization systems, it is valuable to consider the areas in which human cognition is superior to computational models and vice versa. The paradigm of creating a Human-in-the-loop visualization system recognizes that the combination of optimal human cognition and computational models is superior to each separate mechanism [85]. In order to leverage this, the human decision maker needs to be able to influence each individual component of the visualization process. This impacts the visualization pipeline (see Figure 2.2) such that the Mapping phase consists of operations that transform data into representations that are more suitable for the human and that the user needs to be able to change the parameters of each operation to enable an iterative knowledge gaining process.

In almost all cases the human in-the-loop is an expert in the specific application domain, the domain expert, rather than a visualization expert. The importance of this combination and value of visualization in these aspects has well been recognized [119]. This constellation requires the visualization system to be designed in such a way that it is easy and intuitive for the domain expert to understand and control the system and perform the desired tasks. The design of these visualization 
system has undergone many studies and potential tasks have been grouped into varying groups [23], all of which is elaborated on in the next section. However, it is important to acknowledge that these designs rarely succeed on the first try and require iteration, thus requiring user studies and repeated design studies, following the overall design principles of software design [56].

\subsection{Visualization Applications}

Visualization applications are one of the large, and arguably growing, fields of research inside the visualization discipline. As defined by descriptions at the major visualization conferences:"An application paper normally starts with an encapsulated description of a problem domain and the questions to be resolved by visualization, then describes the application of visualization to the task, any novel techniques developed, and how the visualization solution answered the questions posed. Techniques related to a single problem are normally application papers, and evaluation is often limited because many application papers are essentially custom software for a specific problem."

As visualization applications deal with specific needs of userS, these user groups have to be intimately involved with its design and the development. In many cases these are single applications that combine multiple visualization techniques and, thus, amplify the contributions of each constituent component. An example framework for this paradigm is presented by Rungta et al. in their ManyVis system [99].

One widely used technique combines multiple views and enables linking \& brushing between the views. The usefulness of multiview setups was shown by North and Shneiderman [88], whereas Wang et al. provided guidelines for their usage in visualization [123]. This includes specifying different rules, such as the Rule of Diversity, Rule of Complementarity and others.

\subsubsection{Forms of Collaborative Research}

Kirby and Meyer provided an overview of different types of visualization collaborations that can be served by developing an application [50]. In particular with regard to the scientific disciplines that are involved in the project, they highlight three flavors of teams. An interdisciplinary team consists of scientists where there is a discipline gap and thus novel problems are solved by combining techniques from multiple distinct disciplines. Multidisciplinary research solves challenges by tightly coupling techniques from distinct disciplines and thus enables solutions that are not solvable by each discipline alone. Third, intradisciplinary research is performed by collaborators from different sides of the same large discipline and fosters the internal cohesion of the scientific discipline. Placed into the framework put forth by van Wijk, interdisciplinary and intradisciplinary teams would be placed on opposite spectrums of the knowledge gap dimension between collaborators [120]. 


\section{Domain situation}

(1) Threat Wrong problem

$\checkmark$ Validate Observe and interview target users

Data/task abstraction

(1) Threat Wrong task/data abstraction

\section{(iii) Visual encoding/interaction idiom}

(4) Threat Ineffective encoding/interaction idiom

Validate Justify encoding/interaction design

\section{w' Algorithm}

(1) Threat Slow algorithm

Validate Analyze computational complexity

Implement system

Validate Measure system time/memory

Validate Qualitative/quantitative result image analysis

Validate Lab study, measure human time/errors for task

Validate Test on target users, collect anecdotal evidence of utility

Validate Field study, document human usage of deployed system

Validate Observe adoption rates

Figure 2.5: The nested model of visualization application design introduced by Munzner $[84]^{1}$. Image adopted from Munzner.

\subsubsection{Fundamentals of Application Design}

Many models describing the design of visualization applications have been published over the years. One of the more successful models is the nested four-layer model introduced by Tamara Munzner in 2009 that describes the design of visualization applications [84]. Following this model, the design of an application consists of four sequential layers (see Figure 2.5). An error in validity of a layer impacts the downstream layers, similar to the waterfall modeling in software engineering [98]. These layers are the Domain Problem and Data Characterization, in which the visualization designer immerses themselves in the target domain and vocabulary in order to characterize the workflow of the tasks. In the Operation and Data Type Abstraction layer, this knowledge is converted into a more generic computer science description of the challenges and operations that are required by the 
desired workflow of the domain expert. These operations are then converted into visualization components in the Visual Encoding and Interaction Design phase in which either novel visualization techniques are designed or previously published techniques are combined to solve the user's particular problem. In the last step, the Algorithm Design all desired visual encodings are implemented to create the final system, solving potential technical challenges.

Each layer in this nested model has unique threats to its validity that influence subsequent layers. For example, a threat to the Domain Problem layer would be a mischaracterization of the domain expert's desired workflow. Even if the subsequent steps are performed successfully, the designed application will be unable to fulfill the expert's wishes and thus ultimately fail. However, some of the validation of outer layers can only occur after the downstream layers have already been validated leading to a cascading error if the downstream layers' validation fail.

This nested layers and thread model of application design was later improved by Meyer et al., which included more fine-grained subdivision within each layer by introducing transactional blocks that can be identified in each layer and guidelines that describe relationships between blocks [78]. Using this framework, it is possible to characterize the design process of an application system on an abstract level, allowing the designer to analyze and compare different application designs.

\subsubsection{Classification of Visualization Tasks}

One of the layers in Munzner's nested model is the "Operation and Data Type Abstraction" layer. Placed on the border between the abstract description of the scientific domain and the concrete details of visualization methodologies, this layer lends itself perfectly to a taxonomical classification. Like in other areas, the many proposed taxonomies across the subfields of information visualization, scientific visualization, an visual analysis show a high degree of similarity. While concrete examples for each subfield might differ, the resulting taxonomies are similar, which points again to the fact that these subfields have more in common than what separates them. In many of these taxonomies there exist a hierarchy of tasks, where high-level tasks such as "confirm hypothesis" are decomposed into groups of low-level tasks, such as "select" or "filter". Taxonomies then combine tasks with different granularity to attempt a full description of the tasks required by a specific visualization system. As specified by Brehmer et al.: "Low-level classification systems often provide a sense of how a task is performed, but not why; high-level models are the converse. Our focus on multi-level descriptions of visualization tasks is intended to close this gap [...]" [22].

One of the earliest taxonomies was produced by Shneiderman in 1996 [108], who introduced a task taxonomy that focusses on the type of the data, such as number of dimensions, static and temporal data, or organization. It then suggests seven high-level tasks (overview, zoom, filter, details-on-demand, relate, history, and 
extract) that describe the kind of the operations users of a visualization system might want to apply to their data. In the same paper and using the same task names, Shneiderman also coins the visualization mantra: "Overview first, zoom and filter, then details-on-demand".

Building on the work by Shneiderman, Schulz et al. create a design taxonomy that is a five dimensional design space that describes individual abstract visualization tasks along the dimensions of goal, means, characteristics, target, and cardinality [104]. The goal, providing the objective or the intended target audience is important for individual tasks as well as the visualization as a whole and is revisited in greater detail in Section 2.4.4. The means define the methods of achieving a specific goal and perscribe the subdivision into smaller tasks. The characteristics specify aspects of the data that the task aims to reveal. The target describes the kind of relations that are investigated and the cardinality characterizes the scope of a visualization task with regard to the data. In addition, Schulz et al. highlight the important distinction between analyzing tasks from a visualization design perspective as visualization consisting of applying tasks to the right data ("Data + Task = Visualization") and the evaluation standpoint asking the question which tasks are most appropriate for a particular set of data and visualization techniques ("Data + Visualization = Task"). Similar to the design space created by Schulz et al., a similar description was provided by Rind et al. with the proposed "task cube", which uses a three-dimensional design space that uses Abstraction, Perspective, and Composition as the defining axes. In this work, they also provide a survey of abstract objective and action categorizations and how each fits into their task cube classification.

Another typology of visualization tasks was proposed by Brehmer et al. that focusses on "why the task is performed, how the task is performed, and what are the task's inputs and outputs" as a low-level distinction [22]. Each of the "Why", "How", and "What" parts of their typology consists of subclasses, one of which is way users are consuming a visualization. They identify three context for consuming visualizations: "Present", "Discover", and "Enjoy", which will be discussed in greater detail in the following section.

\subsubsection{Visualization Application Categories}

Potential categorizations of visualization applications have been suggested on several occasions. One such categorization of is the distinction between explorational and presentational use cases, which was put forward by, among others, van Wijk [119]. It centers on the realization that visualization has to be aimed at different audiences and must adapt accordingly in order to be effective. Not taking the intended audience into account, including their prior knowledge and expectations, is a thread to the first layer of Munzner's model and thus invalidates the entire application. 


\begin{tabular}{|c|c|c|c|c|}
\hline Exploration & Analysis & \multicolumn{2}{|c|}{ Communication } & \\
\hline Exploratory Analysis & Confirmatory Analysis & \multicolumn{2}{|c|}{ Presentation } & [49] \\
\hline Exploratory Analysis & Confirmatory Analysis & \multicolumn{2}{|c|}{ Presentation } & [104] \\
\hline \multicolumn{2}{|c|}{ Exploration } & \multicolumn{2}{|c|}{ Presentation } & [119] \\
\hline \multicolumn{2}{|c|}{ Discover } & Present & Enjoy & {$[22]$} \\
\hline
\end{tabular}

Table 2.1: Relationships of previous visualization application categorizations by Keim et al. [49], Schulz et al. [104], Brehmer et al. [22], van Wijk [119], and the definitions used in this work.

Visualization applications can be categorized by their intended usage and target audience irrespective of their domain. As van Wijk states: "The main use cases for visualization are exploration (where users do not know what is in the data), and presentation (where some result has to be communicated to others)" [119]. It is reasonable, however, to further subdivide the exploration case into visualization systems that are used for an initial hypothesis generation and systems that are used for the repeated verification of hypotheses on different but similar datasets. Keim et al. define the groups "exploratory analysis", "confirmatory analysis", and "presentation" for these categories [49]. Schulz et al. used the same three goals as one of their five dimensional design space for visualization tasks in which the "Exploratory analysis is concerned with deriving hypotheses from an unknown dataset. It is often equated with an undirected search", the "Confirmatory analysis aims to test found or assumed hypotheses about a dataset. In analogy to an undirected search, it is sometimes described as a directed search", and the "Presentation deals with describing and exhibiting confirmed analysis results" [104]. Brehmer et al., on the other hand, use the terminology "Discover" for the first two categories and "Present" and "Enjoy" for the third categories [22]. In this thesis, the categorization by Keim et al. and Schulz et al. are used with the more succinct names Exploration, Analysis, and Communication instead. Table 2.1 provides an overview and a mapping of the definitions by Keim et al., Schulz et al., Brehmer et al., van Wijk, and the following definitions using in this work.

Exploration A visualization application designed for Exploration is targeted towards the initial information gathering and hypothesis generation phase, what van Wijk states as "where users do not know what is in the data". Applications in this category are dominated by a large number of supported features that can be used by the domain expert to dissect their datasets, where the exact result is only vaguely known a priori and unexpected results and discoveries are desired. Visualization applications of this type usually provide tools to combine a large number of high-level tasks that support the user in exploring and analyzing the available data [70]. Streamlined interaction techniques are generally not feasible as it is a priori unknown which aspects of the visualization should be optimized. 
The majority of publications that describe visualization applications are in this category. An example of this is the work by Ferreira et al., which provided an application with tools to analyze New York City taxi data in order to find and form hypotheses about urban transportation [40].

Analysis The second category of applications, Analysis, is also covered by the "where users do not know what is in the data" part of van Wijk's characterization and corresponds to the "confirmatory analysis" of Keim et al. and Schulz et al. In this case a prior hypothesis about the data already exists and the application is designed specifically to let the domain expert answer a narrow question about the data. This category is distinguished by repeated usage of the application on different datasets of the same kind. Applications and tasks in this category greatly benefit from design iterations between the application designer and the domain expert that lead to more effective workflows. The specialization is applied both to the tasks that a visualization system needs to support, as well as the Abstract Visualization Objects that are displayed, which ought to be tailored to the particular hypothesis under consideration. An example for this is the work by Kumpf et al. in which they present a visual analysis application for use with ensemble weather simulations. The tools enables the domain experts repeated analyses of ensemble weather simulations and gather insight into data uncertainty that arises from the use of ensemble simulations [55].

Communication The third category is Communication in which a visualization application is used to disseminate tested and confirmed hypotheses to a wide audience. There are different situations in which visualization applications can be used to communicate scientific findings. In most cases, the target audience's attention is focussed on the visualization, which Schulz et al. coin the "Present" goal [104]. The audience can be in the same domain as the expert, in which case the visualization is used in their own publications or grants to communicate their findings in a more compelling way, or the audience might be the general public, in which case the wider public audience is exposed to the confirmed hypothesis for public outreach. The cases where the audience is not consciously aware of the visualization is covered by the "Enjoy" goal of Schulz et al.'s design space in which case the goal of the user is not to verify or falsify a hypothesis, but rather stimulate curiosity in the topic of interest and enable future exploration. These applications are often used for storytelling purposes, in which "the data analyst uses visualization for both the exploration/analysis and the presentation. However, the way it is used can be very different, the choice of technique will differ, as does how much and which data is shown." Kosara and Mackinlay [53]. They also note that "Visualization researchers often tacitly assume that the tools used for analysis are usable for presentation just as well as for their original purpose. We believe that to be a very limiting assumption, however.". In addition, this category also spans 


\section{Chapter $2 \cdot$ Introduction}

applications that are specifically designed for usage by the general public, which in general do not possess the same amount of knowledge about a topic of interest or visualization design. This places further constraints on the design of visualization applications. An example of this class of applications is the work by Jönsson et al., which provided a method to enable museum visitors to design potentially complex transfer functions through the use of an intuitive interface [48]. 


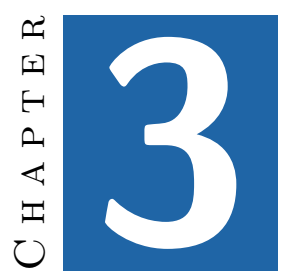

\section{Visualization Application Design (contributions)}

This chapter describes the contributions of the papers that are included in this thesis and the partition of papers into five different application domains. The subsequent sections contain each a description of the problem domain and then elaborate on the contributions of the included papers.

Finite Element Models. Paper A deals with algorithmic challenges to efficiently render non-linear finite element models, in this case exemplified on a high-resolution simulation of stress tensors inside a human heart muscle, a deformation model of the breast muscle, and a simulation of the muscle fibers in the tongue (Section 3.1). Deep Brain Stimulation. Paper B describes an application system that is targeted to be used in deep brain stimulation interventions in order improve the placement of electrodes using a fusion of multiple data modalities in realtime (Section 3.2).

Urban Search \& Rescue. Paper C, Paper D, and Paper E portray the collaborative work of designing a visualization system to support urban search \& rescue operators and rescuers during the reconnaissance phase of partially collapsed buildings. The system utilizes acquired 3D point cloud data as the basis for a path suggestion algorithm, whose results are presented to the expert user for a human-in-the-loop decision support of optimal building exploration in search for victims. (Section 3.3)

Space Weather. The work presented in Paper F describes a visualization application used to compare simulations and real world observations in the context of space weather. Space Weather is the collective term for the study of the overall 
plasma conditions in our solar system and how they impact the Earth, humans, and satellites. (Section 3.4)

Astrophysical Visualization. Paper G, Paper H, and Paper I describe a visualization system called OpenSpace that was designed with a focus on public dissemination of astronomical and astrophysical phenomenæ. (Section 3.5)

Each of the sections provide a short introduction into the domain and then elaborate on the work that has been done in the respective papers.

\subsection{Finite Element Models}

The work of Paper A describes the creation of an algorithm to efficiently render non-linear finite element models that traditionally require expensive calculations during the rendering phase to be represented correctly. The paper describes an algorithm that utilizes an efficient storage and look-up of precalculated rays, thus improving the rendering performance by about an order of magnitude.

\subsubsection{Domain and Scientific Problems}

Finite element models (FEM) methods are used extensively in a large number of fields, such as engineering, construction, or biology, as an approach to solve complex problems numerically by separating the problem domain into a finite number of cells, each with their own local coordinate system, over which the numerical simulation can be performed independently and efficiently. Two coordinate systems are associated with each element; the location and deformation of an element is specified in Cartesian world coordinates, whereas the computed values of the element are represented in a material coordinate system $\xi$ which, in most cases, is not Cartesian but can be of an arbitrary geometry that simplifies the underlying computation (see Figures 3.1(a) and 3.1(b)). The vertex coordinates of each element are expressed in either coordinate system and connected by a bilinear transformation; in many cases, however, an analytical solution does not exist for arbitrary coordinates inside the element and the transformation involves computationally expensive iterative solvers, such as the Newton method. For more information on finite element models can be found in the book by Bathe and Wilson [5].

This work was mainly developed focussing on a simulation of a human heart that calculates the stress tensor at each location during the cardiac cycle. By comparing the results of healthy and abnormal hearts, it becomes possible to detect structural defects before they manifest $[131,132]$. Traditionally, direct volume rendering techniques were not capable of visualizing these datasets and instead isosurfaces or glyphs have been used [129].

When performing direct volume rendering on the FEM dataset, view rays for each pixel are defined in world coordinates and are straight lines in world coordinates. 


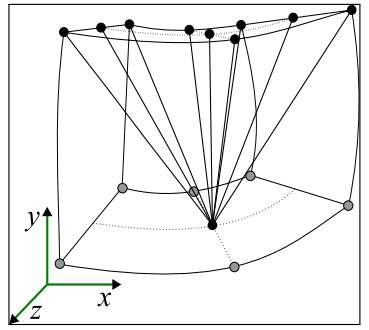

(a) Rays and element geometry shown in Cartesian world space.

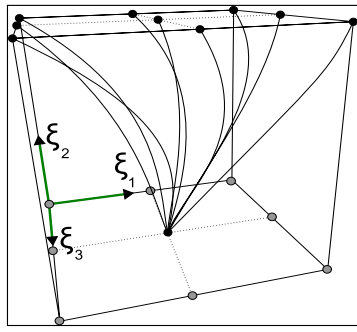

(b) Rays and element geometry shown in material space.

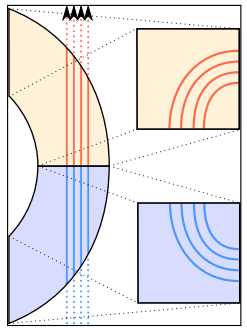

(c) Converting coordinate systems turns straight viewing rays into curved rays.

Figure 3.1: Transformation between world coordinates and material coordinates for a set of viewing rays when viewing the element geometry and the viewing rays from the world (a) or the material (b) coordinate system.

For each element that is intersected by a ray, all rays have to be converted into the material space in order to be able to sample the values, which are given in $\xi$ material space. Figure 3.1(c) shows an example of the ray transformations from a Cartesian world space into a bicubic-linear material space. Converting each sample point from Cartesian space into material space using an iterative solver is prohibitively expensive for real-time use.

\subsubsection{Application Requirements}

For each viewing ray, a large number of sampling points have to be retrieved for a correct front-to-back composited image that minimizes sampling artifacts. The additional coordinate transformation during the data access becomes a bottleneck in the case of non-linear FEM datasets that reduces rendering speeds to non-interactive frame rates. Paper A describes an algorithm that utilizes a precomputation step to cache a reduced set of possible rays that are then used in the rendering step to efficiently access the data, resulting in a $15 \times$ performance gain, relative to straight-forward GPU implementations, which in turn, are an improvement of 2 to 4 orders of magnitude compared to CPU implementations [64].

\subsubsection{Algorithm}

In order to improve the performance of the rendering, the expensive coordinate transformations have to be computed offline prior to the rendering and then retrieved efficiently. The algorithm described in Paper A utilizes a Catmull-Rom spline-based ray representation that can be efficiently stored and retrieved at rendering time [26]. A number of approximation steps are performed in order to increase the similarity between different rays and represent these by a reduced 

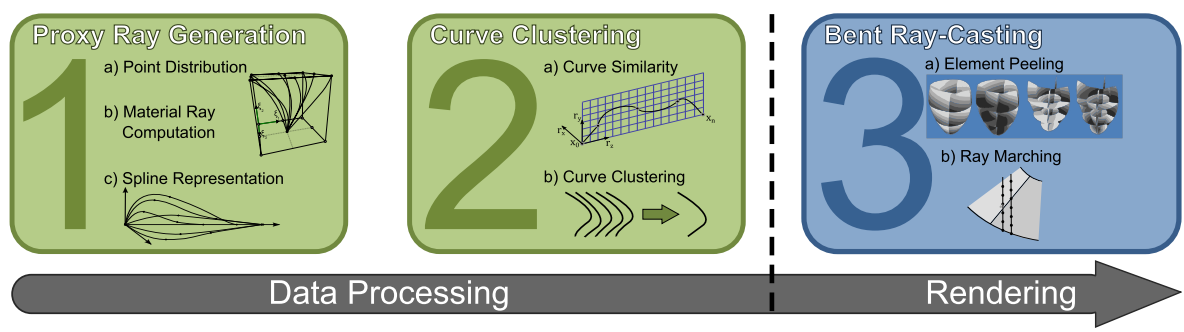

Figure 3.2: The workflow employed in the finite element model volume rendering algorithm. The first two steps are precomputing potential ray paths through the volume that increase the performance during the third step.

set of proxy rays, which drastically reduces the number of rays that need to be stored and still maintain a high quality rendering. Figure 3.2 shows the algorithm's workflow in detail, with the first two steps being precomputed once for each finite element model in order to improve the performance of the third real-time rendering step. The algorithm's total of five steps are described in the following sections:

Point Generation A fixed number of points are generated for all faces of each element. For each point, a proxy ray is created to every other point in the same element but not the same face.

Ray computation The proxy rays for each pair of points are sampled using a fixed sampling rate that is independent from the rendering sampling rate. For each sample, the expensive coordinate transformation is performed).

Curve Similarity Through renormalization and alignment, the similarity between all proxy rays is improved without losing information.

Curve Clustering Using k-means clustering, a representative subset of proxy rays is created. Each original entry-exit point pair references its new representative path.

\section{Rendering}

For each viewing ray, the proxy rays are used to sample the correct locations in material space without the need for the expensive coordinate transformation.

The following two sections describe the curve clustering and the rendering; the point generation and the ray computation steps are described in Paper A.

\section{Curve Clustering}

A sampling of the $|F|$ faces of the $|E|$ elements of the model using $n$ points per face results in $r_{t}=\left(n^{2} \cdot|F|\right)^{2} \cdot|E|$ proxy rays. Without further reductions this number 


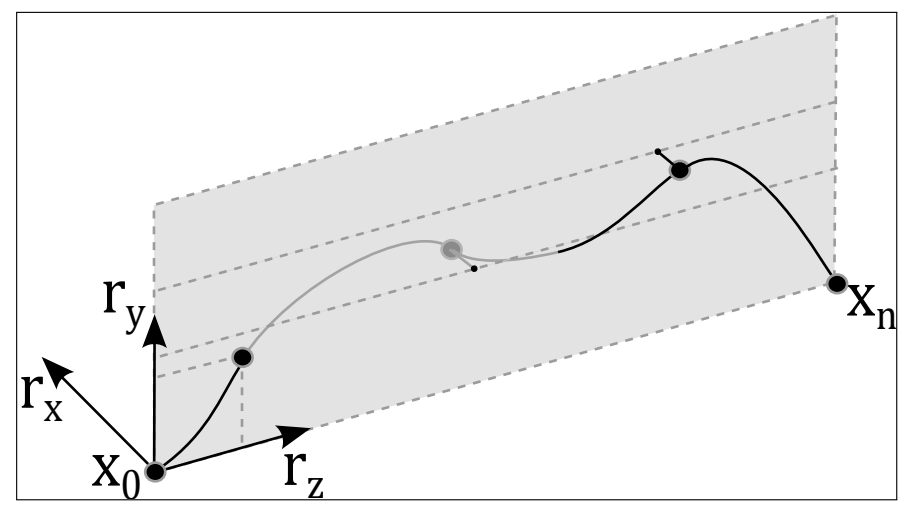

Figure 3.3: Increasing the similarity between splines through a lossless conversion into a common coordinate system. Rigid transformations move the first point to $(0,0,0)$, the last point to $(0,0,1)$ and the first non-collinear point into the $y z$ plane.

is prohibitively large to store all precomputed rays on the GPU. However, since there is a potential for a high degree of similarity between rays due to symmetries in the finite model elements and bounded ray complexity as each ray has to stay inside the boundaries of an element. Thus, it is possible to utilize a clustering algorithm to reduce the number of representative proxy rays necessary during the rendering without introducing large errors.

The first step is to increase the similarity between proxy rays while maintaining the ability to uniquely reconstruct the final ray. All rays are rotated and stretched such that the first point $P_{1}$ is equal to $(0,0,0)$ and the last $P_{n}$ control point is equal to $(0,0,1)$. Then, all control points are rotated by a rotation angle $\theta$ around the $z$ axis such that the first point $P_{i}$ that is not collinear with $P_{1}$ and $P_{n}$ is in the $y z$ plane. Figure 3.3 shows the results of these transformations on an example ray. The translation and the scaling are undone during the rendering step without storing additional information, as the entry and exit points (and their distance) are known. Only the angle $\theta$ needs to be recorded for each proxy ray.

For the clustering of the splines, the k-means [46] algorithm is used because of its stability and ability to deal with values of an arbitrary number of dimensions. By adapting an idea from Abraham et al. [1], the clustering is performed directly on the control points of the Catmull-Rom splines. The distance metric for the clustering uses the area between two curves as approximated by a Riemann sum of triangles connecting the proxy rays, sampled at a high frequency (see Figure 3.4). For two proxy rays $a$ and $b$, and their $n$ sampled points $\mathbf{a}_{\mathbf{1}}, \ldots \mathbf{a}_{\mathbf{n}}$ and $\mathbf{b}_{\mathbf{0}}, \ldots \mathbf{b}_{\mathbf{n}}$ with $\mathbf{a}_{\mathbf{1}}=\mathbf{b}_{\mathbf{1}}, \mathbf{a}_{\mathbf{n}}=\mathbf{b}_{\mathbf{n}}, \exists i \in[2, n-1]: \mathbf{a}_{\mathbf{i} x}=0$, and $\exists j \in[2, n-1]: \mathbf{b}_{\mathbf{j}_{x}}=0$ due to the transformation performed in the last paragraph, the similarity metric is defined as: 


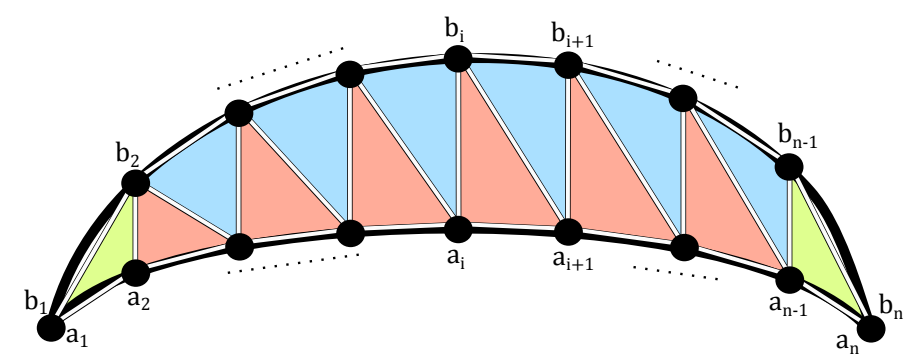

Figure 3.4: A representation of the metric that is used in the k-means clustering algorithm, approximating the area between the two rays by a sum of the triangle areas.

$$
\begin{aligned}
d(a, b)= & \frac{1}{2}\left(\left\|\overline{\mathbf{a}_{\mathbf{1}} \mathbf{a}_{\mathbf{2}}} \times \overline{\mathbf{a}_{\mathbf{2}} \mathbf{b}_{\mathbf{2}}}\right\|+\right. \\
& \sum_{i=2}^{n-2}\left\|\overline{\mathbf{a}_{\mathbf{i}} \mathbf{a}_{\mathbf{i}+\mathbf{1}}} \times \overline{\mathbf{a}_{\mathbf{i}} \mathbf{b}_{\mathbf{i}}}\right\|+\left\|\overline{\mathbf{b}_{\mathbf{i}} \mathbf{b}_{\mathbf{i}+\mathbf{1}}} \times \overline{\mathbf{b}_{\mathbf{i}+\mathbf{1}} \mathbf{a}_{\mathbf{i}+\mathbf{1}}}\right\|+ \\
& \| \overline{\mathbf{a}_{\mathbf{n}-\mathbf{1}} \overline{\mathbf{a}}_{\mathbf{n}}} \times \overline{\left.\mathbf{a}_{\mathbf{n}-\mathbf{1}} \mathbf{b}_{\mathbf{n}-\mathbf{1}} \|\right)}
\end{aligned}
$$

Using this metric, all proxy rays are then clustered into $k$ representatives. For each proxy ray, the identifier of the representative, as well as the angle $\theta$ are stored.

\section{Rendering}

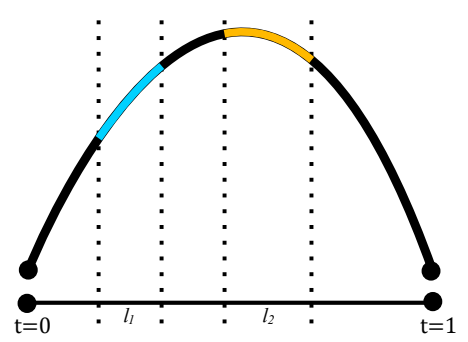

During the rendering step of the algorithm, the geometries for all elements of the finite element model are rendered. Their coordinates are mapped to the RGB channel, as described in the DVR technique by Krüger and Westermann [54], and the face identifier and element identifier are encoded in the transparency channel. The entire scene is then rendered in multiple passes, employing a modified depth peeling approach as

Figure 3.5: The desire to uniformly sample the view ray in world space requires an arclength parametrization of the proxy ray. described by Everitt [38] for each rendering pass. In every pass, the entry and exit point geometries are rendered which trigger the fragment shader of the GPU that performs the rendering as described in the rest of this section. As all of the finite elements are colocated, z-fighting artifacts can occurr during the render passes (see Figure 3.6).

For each of the peeling steps, the color information for every pair of fragments is used to access entry and exit points, the face, and element ID. Using this 


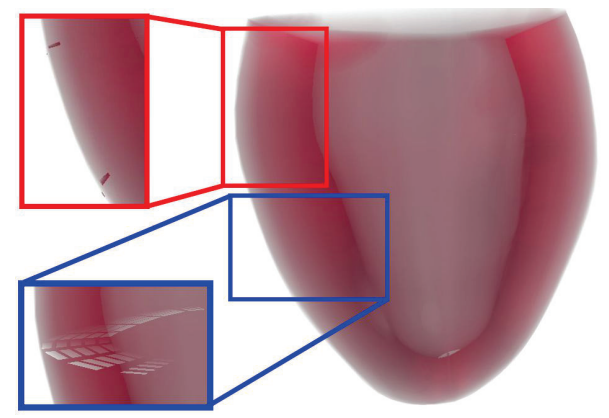

Figure 3.6: The insets show potential rendering artifacts when applying the depth peeling algorithm to finite element methods due to z-fighting.

information, the cluster ID and the angle $\theta$ of the proxy ray that is closest to the fragment pair is retrieved. The previous transformations of the proxy ray are undone by scaling the proxy ray by the distance between the entry and exit points in world coordinates, translating and rotating the ray such that $P_{1}$ coincides with the entry point and $P_{n}$ coincides with the entry point, and then rotating the proxy ray around the axis connecting $P_{0}$ and $P_{n}$ by the stored angle $\theta$.

The ray marching then interpolates along the Catmull-Rom spline to retrieve the correct sampling value in material space. The step size $h$, however must not be constant in the material space $\xi$ as this would lead to a non-uniform sampling in world space (see Figure 3.5). Therefore, the spline interpolation parameter $t \in[0,1]$ has to be converted into an arclength parametrization such that the sampling in material space becomes non-uniform to make the world space sampling uniform instead [42]. A second subtle error occurs at the boundary between elements and is exemplified in Figure 3.7. Starting the ray marching at the beginning of each element would also lead to a non-uniform sampling across element boundaries and thus introduce rendering artifacts. For this reason, the remaining distance between the last sample and the exit

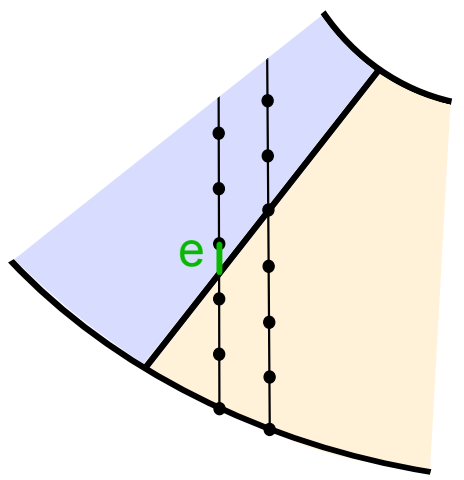

Figure 3.7: Special border handling is required between elements, as a naïve implementation would oversample the boundary. point is stored at the end of the ray marching and this distance is used to offset the first sampling point in the following element, similar to the method employed by Ljung et al. [66].

A low sampling resolution of proxy rays introduces rendering artifacts as the 


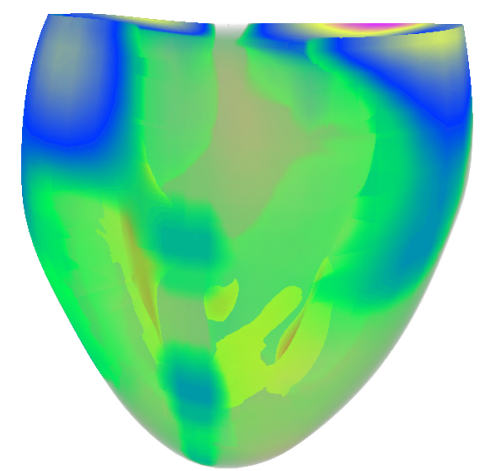

(a) Using no inter-ray or intra-ray interpolation.

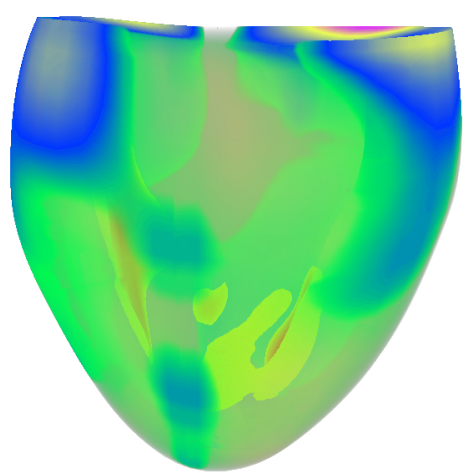

(b) Using inter-ray and intra-ray interpolation.

Figure 3.8: A volume rendering of the heart dataset using $5 \times 5$ rays per face for each element showing the difference between the interpolation schemes. Particularly with long rays, on the left and the right side of the heart, the errors are more pronounced.

same proxy rays will be used for a large area of the face; similar to nearest neighbor interpolation. In order to mitigate these artifacts, we introduce a bilinear interpolation of control points of the four closest proxy rays and perform the ray marching along the spline generated by these interpolated control points. This interpolation method is called inter-ray interpolation.

A second mitigation of low sampling rate utilizes the fact that the precomputation step considers the ray between entry point $e$ and $f r_{e f}$ independently from the ray $r_{f e}$. This makes it possible to sample $r_{e f}$ with the sampling parameter $t$ and $r_{f e}$ using $t^{\prime}=1-t$ and averaging the results before sampling the volume. This method is called intra-ray interpolation. Figure 3.8 shows the difference using the two interpolation methods, where $5 \times 5$ rays per face for each element were generated.

\subsection{Deep Brain Stimulation Interventions}

Paper B describes the work in developing a medical visualization application for deep brain stimulation (DBS) intervention support in collaboration with physicians at the St. Barbara Hospital in Hamm, Germany. This system was designed to support the brain surgeon during an electrode placement surgery in order to achieve a higher precision and thus a higher probability of a positive outcome for the patient. 


\subsubsection{Domain and Scientific Problems}

Deep Brain Stimulation (DBS) is a form of medical intervention that targets, among others, patients afflicted with Parkinson's Disease or other forms of essential tremors. During these interventions, electrodes are inserted into patient's brain that stimulate the surrounding regions by using an electric field. Depending on the exact location and geometry of the electrode, different regions of the brain are stimulated, which has the potential to inhibit some of the debilitating effects of these tremors [44]. One target region for the treatment of tremors are the patient's subthalamic nuclei (STN), which are two structures with the size of a few millimeters located deep in each hemisphere of the brain [6, 94]. Using traditional imaging techniques such as CT or MRI, the exact location of an individual patient's STN can be difficult to determine [110]. This procedure is further complicated as small deviations in the electrodes' locations will excite other parts of the brain that can lead to side effects, such as memory loss or speech impairment.

Microelectrode Recordings (MER) have been developed to augment imaging modalities and make use of a cluster of recording electrodes that are capable of detecting the electric activities in the brain [57]. The amplitude and frequency of the electric fields measured by the electrodes correlate with specific brain regions and can thus be used to determine whether the electrodes are in the correct location [7]. Currently, the results of the MER are reviewed by the surgeon on loudspeakers in the operating room. Aside from the obvious drawbacks of a limited auditory channel, a limited echoic memory, and potential background noise, a big challenge is the surgeon's mental separation between the spatial location of the electrodes and the results of their measurements, requiring the surgeon to keep a mental model of the brain regions and comparing those to a standard atlas in their head. The same argument applies to the absolute location of the electrode cluster as well as the relative position of the electrodes inside the cluster.

A DBS intervention is performed in three distinct phases. In the first phase, the planning phase, the surgeon plans the operation at his workstation by locating and segmenting the most probable location of the STN using preoperative CT and MRI scans. This information is used to plan an optimal access channel which evades important sensitive brain regions and selects the optimal location for the electrode to effect the STN [24]. In the second phase, the recording phase, the patient is in the operating room with a stereotaxic frame providing mounts for the instruments being fixed to their head. The frame is visible on all scanning modalities and thus allows for a fixed-body transformation between the patient's head and the operating room. The MER electrode cluster is inserted into the patient's brain along the preplanned access path until they have reached the predicted STN location. During this phase, the measurements of the electrodes are used to discriminate the different brain regions and verify that the STN has been reached. If the electrodes correctly identify the location of the STN, the electrodes' position along the access path is noted and their position relative to the stereotaxic frame is verified using bi-planar 
X-ray scans. After this verification, the electrodes are retracted. In the third phase, the placement phase, the transmitting electrode is inserted into the same location that was determined in the previous phase, which is also verified using bi-planar $\mathrm{X}$-ray scans. The exact placement and the output of the electrode is modified using tests that examine the patient's ability, such as long term memory recall or measuring the amount of tremor. This part of the procedure is challenging as the patient is awake during the entire procedure, which can last up to 10 hours.

\subsubsection{Application Requirements}

Previous methods do not provide the surgeon with a system that sufficiently fuses the available information during the procedure. Preoperative CT and MRI scans, interoperative X-ray scans, the electrode measurements during the insertion, and the final patient tests are all inspected independently and the surgeon has to maintain a mental correlation between all data sources, leading to fatigue, delays, and potential errors. The collaboration partners desired a system that can ingest the access path planned using other sophisticated tools (see Figure 3.12), the various scans ( $\mathrm{T}_{1}$ and $\mathrm{T}_{2} \mathrm{MRI}, \mathrm{CT}$, and X-ray), the MER measurements, as well as the patient tests during the operation and display them in a linked application. Furthermore, a more effective visual encoding of the MER and the patient tests was desired by the surgeons.

There were a number of technical challenges that had to be addressed while designing the system; 1 . An automated classification of the MER signals has to be displayed to the surgeon in context of the preoperative and interoperative CT, MRI, and X-ray scans. 2. A visual representation that enables the surgeon to interpret individual MER signals from the electrodes must be made available. 3 . The system must contain a visual representation of the information gathered during the placement phase of the intervention, making it possible for the surgeon to visually correlate the information from the patient tests and X-ray scans.

Addressing these challenges requires the design of a system combining multiple linked views. The following sections describe these views in greater detail.

\subsubsection{Contextual View}

This 3D view combines preoperational CT and MRI scans with the bi-planar X-rays and the current electrode location (see Figure 3.9). The view contains a multimodal volume rendering that shows the head and brain using $\mathrm{T}_{1}$ and $\mathrm{T}_{2}$-weighted MRI scans, the patient's skull extracted from the CT scan, and the interoperative X-ray scans as orthogonal planes. The user chooses a vertical clipping threshold for $\mathrm{T}_{1}$ scan and a separate threshold for the $\mathrm{T}_{1}$ and the CT scan, making it possible to inspect only the $\mathrm{T}_{2}$ scan of the brain while maintaining the context provided by the $\mathrm{T}_{1}$ and $\mathrm{CT}$ scans. The clipping is performed using the skull stripping algorithm 


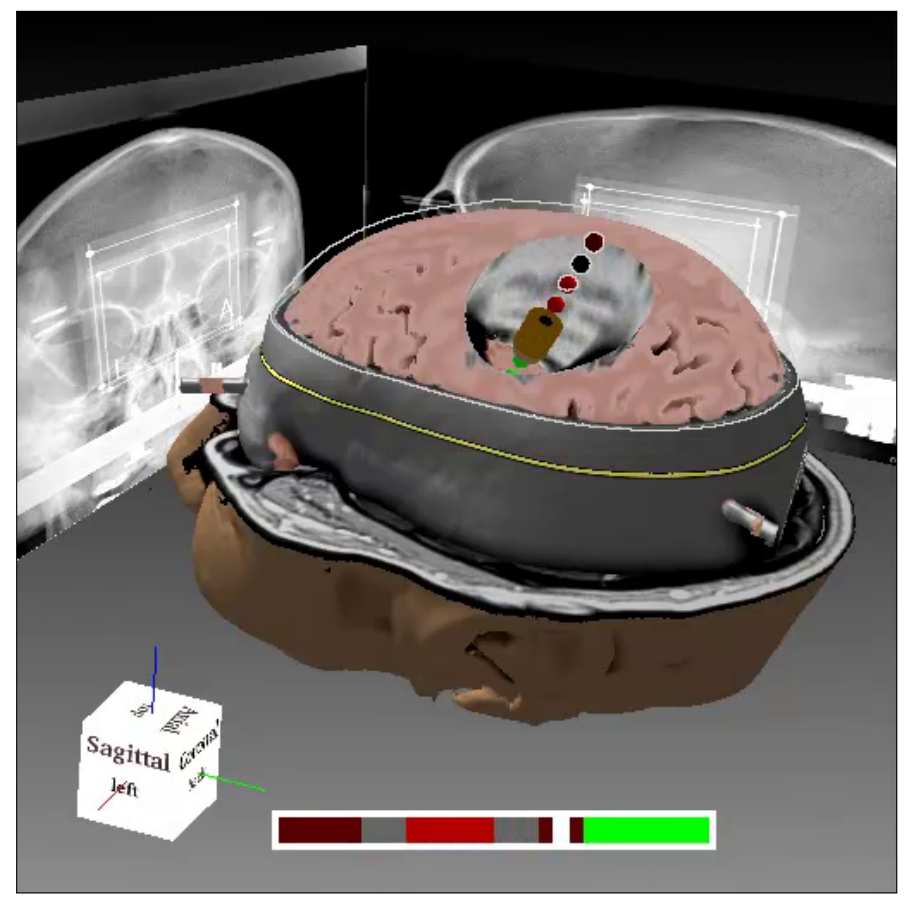

Figure 3.9: The contextual view provides the user with information about the location of the electrode relative to the patient's brain as well as the detected brain regions.

described by Beyer et al. [8]. The outermost layer contains a yellow band that shows the projected depth of the intended target location. The planned access path is removed from the datasets and a single representative electrode is rendered at its location along this path. The electrode provides the necessary spatial relation to the string of beads that are inserted into the path for each successful detection of a brain area by the MER. Additionally, it prevents a dangerous left-right mismatch error that might otherwise occur during the operation in which the electrode is accidentally inserted into the wrong hemisphere. This metaphor has already been used in similar systems when applied to non-human primates et al. [80] and presents the user with a visual representation of the various brain region the electrode has passed. In order to improve the user's depth perception, the view uses a depth darkening effect that was presented by Luft et al. [68]. In addition to the electrode position as recorded by the instruments, this view also shows a reconstructed position of the electrode using the bi-planar X-ray scans which can be used by the surgeon to verify the correct placement position. Lastly, this view also contains a separate horizontal indicator that presents the location of the electrode as well as the detected brain regions to the user without occlusion. 


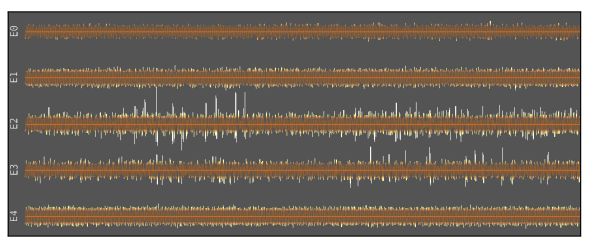

(a) The 2D oscilloscope rendering of the direct electrode measurements. Measurements with low amplitude are deemphasized by using darker colors.

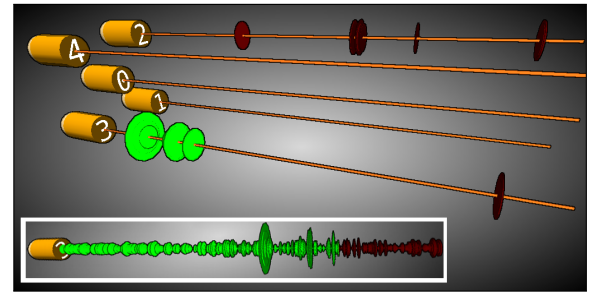

(b) The spatial 3D rendering of the electrode rendering shows their relative spatial location and rendering the high-amplitude measurements of the signal as colored discs.

Figure 3.10: The two rendering methods for displaying the measurements recorded by the MER electrodes. The views show the accurate values as well the relative spatial relation between the electrodes with an abstract representation of the measurements.

\subsubsection{Audio visualization}

The visual representation of the MER measurements is displayed to the user in two separate views. The collaborators in the project used a microelectrode cluster that contains five electrodes, each of which is recording a separate signal from their specific location. Correlating the difference in measurements between electrodes provides information about the borders between brain regions, making it important to register the measurement locations. A combination of two views presents the user with the MER measurements and at the same time provides the spatial mapping between recording and electrode location.

The first view shows an augmented visualization similar to an oscilloscope that presents the direct measurements from the electrodes. As only the amplitude and frequency are relevant to the user during the procedure, we emphasize measurements that exceed a user-defined threshold and deemphasize the values below that threshold (see Figure 3.10(a)). This highlights potentially important measurements and reduces the noise from the low intensity signals.

The second view displays a 3D representation of the electrodes' orientations and their measurements (see Figure 3.10(b)). The camera orientations of this view and the Contextual View are linked such that the mental registration between the two views is not broken. In this view, each electrodes' measurements that exceed a userdefined threshold are shown as concentric discs that start at the electrodes moving away from the base with increasing time. The size of the disc corresponds to the amplitude of the detected signal and thus shows the strength of the measurement. This enables the surgeon to inspect the amplitude and frequency for each electrode in their current spatial location. The color of each disc is determined by the classification algorithm that is used on the beads in the Contextual View, but is 


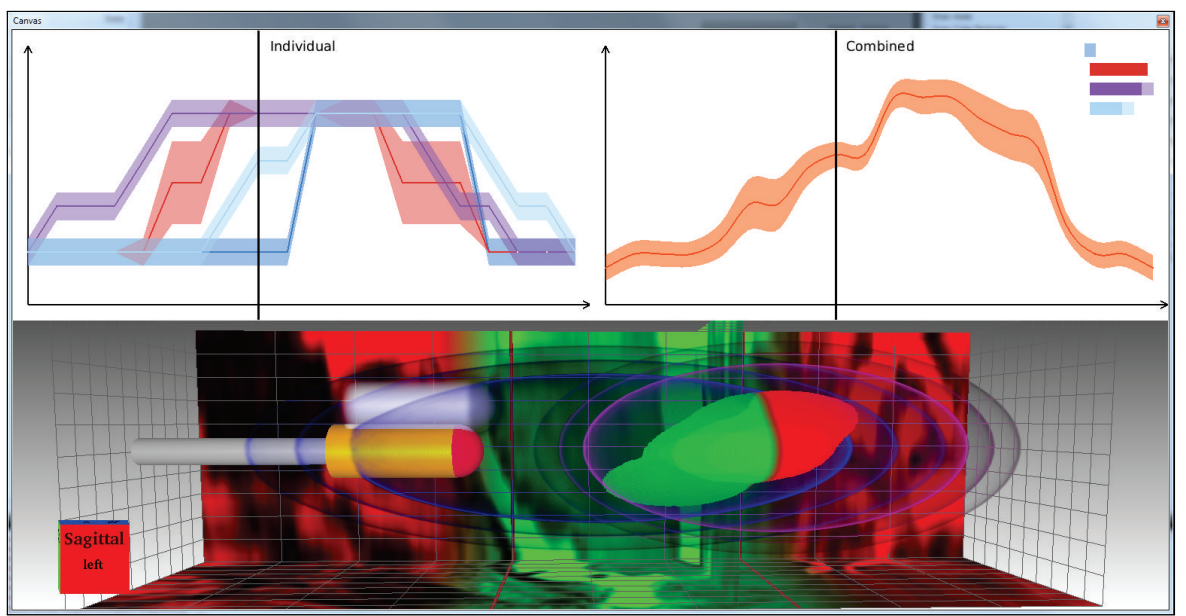

Figure 3.11: The views that show the results of the different measurements performed during the recording phase. The Target closeup (bottom view) contains the segmented location of the STN as well as the results of the different tests at their spatial location. The Placement guide shows the likelihood measurements according to their depth along the access path.

simplified to only distinguish whether the electrode is inside or outside the STN.

\subsubsection{Target closeup}

After determining the potential electrode location, the closeup view, which is centered around the segmented location of the STN, combines all information that is relevant to the surgeon for the final placement of the stimulating electrode (see Figure 3.11). Embedded in this view are the locations of the electrode as determined by the instrument's depth along the access path and the reconstructed location using the biplanar X-ray scans. On demand, the surgeon can further enable the rendering of the MRI $\mathrm{T}_{2}$-weighted MRI scan of the area. In addition, it shows the recorded MER signal results as a red-green overlay on the backside of the bounding box and presents options to add the results of patient tests as additional transparent oval overlays. All values show an estimate of their unknown uncertainty in this view that provide the surgeon with the information for a potential final placement that agrees with most measurements.

\subsubsection{System}

The application resulting from this collaboration combines an enhanced multimodal $3 \mathrm{D}$ volumetric rendering environment with the spatial visualization of electric 


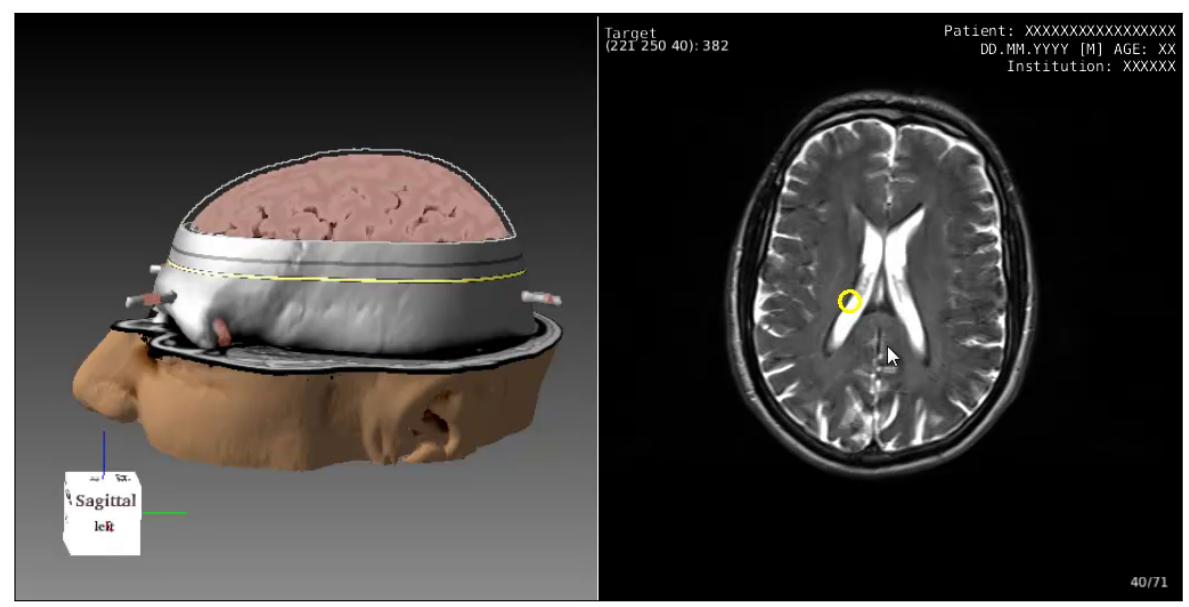

Figure 3.12: This view shows the part of the system that is used to import the results of a different planning tool to determine the entry point for the access path and the intended target location.

measurements that are recording the patient's brain activity during the procedure in combination with patient-specific ability tests. Spatially embedding the measurements with the volumetric information reduces the cognitive load of the surgeon during the surgeon, as this mental link does not have to be performed by the surgeon themself. Furthermore, the system shows the uncertainties of different modalities in a single view, thus enabling the surgeon a comprehensive view and more insight during the procedure.

\subsubsection{Evaluation}

We performed an initial evaluation of the system using a qualitative user study with five neurosurgeons, which all had experience with conducing DBS interventions. Each of the participants watched the usage of the system during the planning, recording, and placement phase. The data for this test case was recording during an operation performed by one of the coauthors of Paper B. Then, each participant answered a questionnaire that used eight of the questions suggested by Martelli et al. for evaluating computer-aided surgery systems [71] with an additional free form text field for comments. For each question, the participants could choose their reply on a 3-point Liekert scale [60]. The feedback from the experts was overall positive, with even the least positive participant agreeing to the majority of provided statements. More information about the evaluation can be found in Paper B. 


\subsection{Urban Search \& Rescue}

The work presented in Paper C, Paper D, and Paper E was developed in the domain of Urban Search $\&$ Rescue (USAR), a field of civil security that involves the location and extraction of victims that are trapped in collapsed buildings. The goal of the work was to design a visualization application in collaboration with the Myndigheten för samhällsskydd och beredskap, the Swedish Civil Contingencies Agency, that expedites the location and extraction of victims in buildings through the use of sensor-equipped autonomous drones.

\subsubsection{Domain and Scientific Problems}

Traditionally, in the case of a partial building collapse in an urban environment, a country's civil contingency agency will perform an USAR operation in order to search the building for potentially injured survivors or victims and perform a rescue of these victims. This operation entails responders to enter the building being directed and coordinated by an incident commander (IC). The major obstacle during these rescue operations is the fact that previous structural plans of the building are no longer usable and new unknown hazardous environments may have been created by the incident. In addition, the viewing distance is usually restricted as debris and smoke impede the responders' progress. During the rescue operation, the IC relies on descriptions of the responders to manually create a map (see Figure 3.13). This map is important for the IC in order to; 1 . optimally coordinate the rescuers as time-to-rescue is the dominating factor in determining victims' survivability; 2 . due to the collapse entire parts of the building might be inaccessible without removing rubble. These voids are prime locations of trapped victims and their detection on an unstructured 2D map is difficult.

The robotics community, for a long time, has been advancing research into drones equipped with sensors and autonomous control in the usage for urban search \& rescue [65] that enable the improvement of the current workflow and creation of a system that supports the IC during a rescue operation. While traversing the building, the rescue robots carry multiple scanners, for example Lidar, heartbeat detectors, or scanners to detect gas leaks. These scans create a 3D point cloud map of the structure used by the IC plan a better path for the responders.

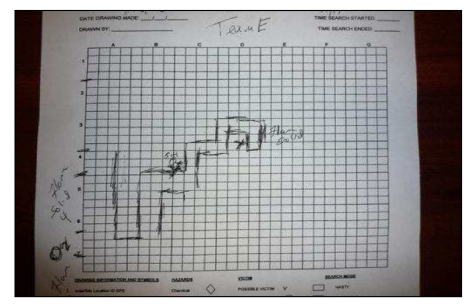

Figure 3.13: A drawn map is the state-of-the-art method for coordinating multiple rescuers.

The point clouds in their native form (see Figure 3.14) are difficult to interpret due to missing occlusion or other visual queues. Furthermore, important aspects for the responder, such as location and dangerous 
environments, the slope of terrain, the existence of overhangs, and the available space for responders are difficult to extract.

Therefore, Paper C, Paper D, and Paper E set

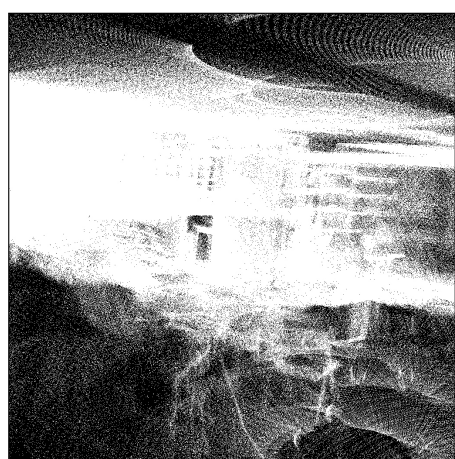

Figure 3.14: A naïve rendering of the point cloud makes it difficult to inspect details in the building. out to make the analysis of the $3 \mathrm{D}$ point cloud data easier for the IC and suggest a variety of paths in order to move the current ad hoc decision process into a higher planning mode. Figure 3.15 shows the workflow of the application that resulted from this. As the data is likely to be noisy and the domain knowledge of the IC is important in the decision making, a fully automatic algorithm is not desirable. Instead a set of suggestions are generated and visualization tools enable the IC to make a good selection out of a set of suggestions.

\subsubsection{Application Requirements}

Paper C, Paper D, and Paper E describe the effort of developing a system that provides the IC with the tools to analyze the acquired point cloud data and that suggests a set of paths together with the visualization tools to analyze the paths and make an informed decision about a path selection. The system meets a set of challenges in order to be beneficial to the IC; 1 . The system must increase spatial awareness and depth perception by allowing for interactive exploration of the collapsed structure. 2. The system must enable the IC to interactively annotate the acquired data to react to changing circumstances. These annotations are potential entry points, points of interest (POI), and the ability to add and remove obstacles from the map, as all of these change during the course of the rescue operation. 3. The system must automatically generate a set of candidate paths for the responders and provide the IC with the tools to inspect the available access paths, compare them, make trade-offs, and select and execute the optimal path. The different paths are generated by changing importance weights on the path computation algorithm that leads to a set of distinct paths covering a multidimensional decision space and includes paths that favor, for example, a more dangerous path over a shorter path. These paths are presented to the IC with decision-making tools in order to select the single path that is then executed.

\subsubsection{Voxel Binning}

The acquired map is an unstructured point cloud (see Figure 3.14) and does not allow for easy interpretation as the individual points are not space-filling and thus provide no depth clues to the user. In order to solve this challenge and provide the 


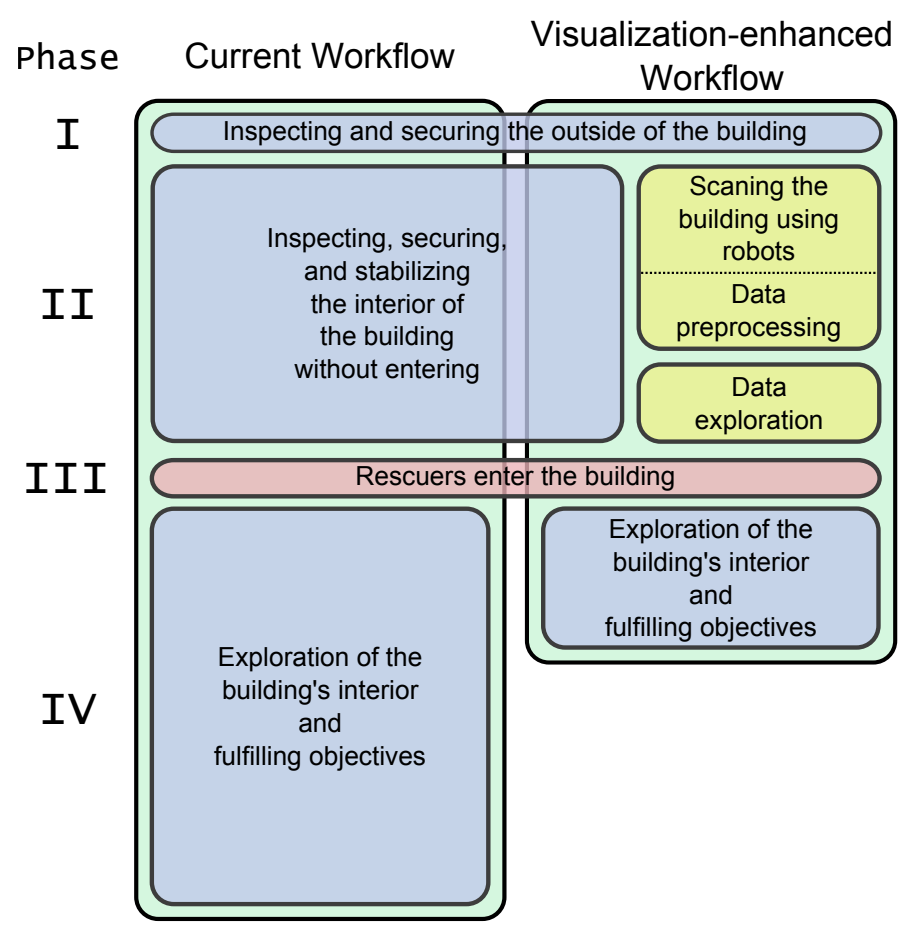

Figure 3.15: Analyzing the currently employed workflow of the rescue operation, it is possible to retrieve full scans of the building during the initial inspection phase during which no human is allowed access to the building. The gathered information can then later decrease the time necessary to explore the building.

user with a familiar view of the building's interior, a binning technique is applied to the point cloud. A voxel grid is created with a user-defined binning size that covers the extend of the point cloud. Each voxel in the grid is marked with the number of points that fall into its extend and only voxels with an occupancy of greater than a fixed value are considered. Figure 3.16 shows an example of the same point cloud binned with voxels of different bin sizes. The size of the voxels depends on the resolution of the scanner that is employed where a larger bin size deemphasizes the noise in the data and a smaller bin size provides higher detail. For the binning, we utilize the widely used Point Cloud Library (PCL) that provides efficient methods to construct out-of-core voxel grids as well as reconstruct surfaces from the data [101]. These grids are then converted into a new point cloud, where only the center point of each voxel is stored.

In addition to the Lidar data that is used to determined whether individual voxels are present in the map, additional scanners or manual input can be used to mark 


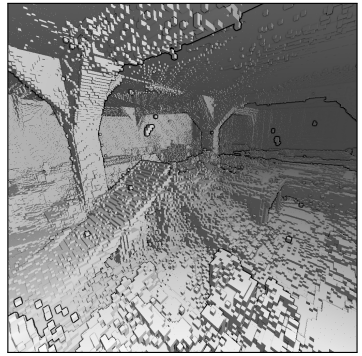

(a) Binning of a dataset with a voxel size of $4 \mathrm{~cm}$.

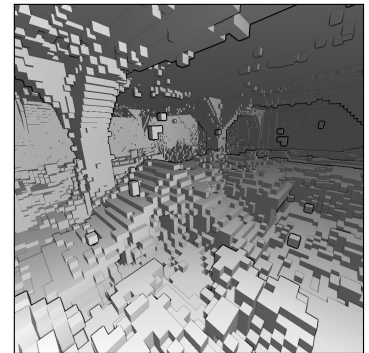

(b) Binning of a dataset with a voxel size of $10 \mathrm{~cm}$.

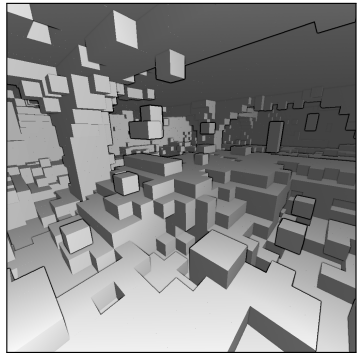

(c) Binning of a dataset with a voxel size of $25 \mathrm{~cm}$.

Figure 3.16: The resolution of the voxel grid has a direct impact on the trade-off between achieved resolution and the amount of sensor noise that is included in the data. Here, a rescue arena from the Jacobs University rescue arena is used.

voxels. These markings can be the location of potential entry points into the structure, the location of victims or other points of interests, or the location, type, and severity of hazardous environments. Based of this information, it is possible to compute derived attributes for the voxels in the grid.

Hazard The distance to the closest voxel that is marked as a hazardous environment.

Support The number of unobstructed surrounding voxels at the same height, given a desired floor support for a human responder of about $40 \times$ $40 \mathrm{~cm}$.

Size The amount of space that is available above the specific voxel indicating whether it is necessary for the responder to walk or crouch over this location.

Normal Determines the orientation of the original point cloud that this voxel covers based on a reconstructed surface model. If the normal has a high angle, the more dangerous it is for responder to traverse.

Occupancy The amount of points in the original point cloud that are covered by the voxel. This provides an indication of the certainty that the voxel is not a scanning artifact.

Overhang Determines whether there is an overhang, which is a potentially unstable structural element above the voxel.

All computed values are stored for each occupied voxels and most are utilized in the path computation to favor or exclude certain areas of the map. It then becomes possible to vary the weight of each value and generate a set of paths that are each 
optimal for the specific weight values but provide the IC with a set of candidate paths from which to choose.

\subsubsection{Path Computation}

In order to perform the path computation, the system uses the widely used $A^{\star}$ algorithm which utilizes a metric or cost function $\mathrm{m}\left(\mathbf{x}_{\mathbf{1}}, \mathbf{x}_{\mathbf{2}}\right)$, which determines the cost or error to move between two nodes $\mathbf{x}_{\mathbf{1}}$ and $\mathbf{x}_{\mathbf{2}}$ in a graph [45], and a heuristic $\mathrm{h}\left(x_{1}\right)$, which determines the approximate remaining distance to the goal. $\mathrm{A}^{\star}$ is an informed algorithm that, for a given graph and metric, finds the optimal path between two points through an exhaustive greedy search of the graph. In the case of the voxel grid, each voxel is treated as a node with a theoretical maximum of 26 edges, if all of surrounding voxels are filled. The interested reader is referred to the book by Russel and Norvig for a full description of the $A^{\star}$ algorithm [100].

The only requirement for the heuristic, $\mathrm{h}\left(\mathbf{x}_{\mathbf{1}}\right)$, is that it is admissible, which means that it never overestimates the distance between $\mathbf{x}_{\mathbf{1}}$ and the goal. For the path computation in the voxel grid, the $L^{2}$-norm is used as an heuristic. It is admissible as the movement along the voxels follows the $L^{1}$-norm and is thus guaranteed to be larger than $\mathrm{h}(\mathbf{x}) \forall \mathbf{x}$.

Cost function. The design of the cost function $\mathrm{m}\left(\mathbf{x}_{1}, \mathbf{x}_{2}\right)$ is of vital importance for $A^{\star}$ as it defines the resulting path's optimality. This system uses a cost function that consists of a number of additive, weighted sub-functions. Thus, the optimal path will be different for each combination of weighting factors. This enables the construction of a multidimensional search space $\mathcal{P}$ where each element $\mathbf{w} \in \mathcal{P}$ is a set of weights and thus associated with a single optimal path through the voxel grid. In the current system, $\mathcal{P}=\mathbb{R}^{8}$ with parameters for the hazard $w_{h}$, size $w_{s}$, normal $w_{n}$, normal threshold $\varphi$, support $w_{\text {sup }}$, desired support $n$, overhead $w_{o}$, and occupancy $w_{\text {occ }}$. The final cost function is then defined as:

$$
\begin{aligned}
m\left(\mathbf{x}_{1}, \mathbf{x}_{\mathbf{2}}\right)= & L_{2}\left(\mathbf{x}_{\mathbf{1}}, \mathbf{x}_{\mathbf{2}}\right)+w_{h} \cdot \operatorname{hazard}\left(\mathbf{x}_{\mathbf{2}}\right)+w_{s} \cdot \operatorname{size}\left(\mathbf{x}_{\mathbf{2}}\right)+ \\
& w_{n} \cdot \operatorname{normal}\left(\mathbf{x}_{\mathbf{2}}, \varphi\right)+w_{\text {sup }} \cdot \operatorname{support}\left(\mathbf{x}_{\mathbf{2}}, n\right)+ \\
& w_{o} \cdot \operatorname{overhead}\left(\mathbf{x}_{\mathbf{2}}\right)+w_{\text {occ }} \cdot \operatorname{occupancy}\left(\mathbf{x}_{\mathbf{2}}\right)
\end{aligned}
$$

Path classes. When sampling $\mathcal{P}$ sufficiently high with about $10^{7}-10^{9}$ samples, it became clear that many of the sampled weights resulted in only a small number of representative paths with only minor or no variation. We therefore grouped the computed paths into classes by comparing the ordered list of voxels that uniquely define a path. For a user-defined threshold $a$, paths are considered equal if at most $a$ subsequent voxels in the ordered list are different. This reduces the number of paths that need to be considered during the rendering drastically and thus increases performance and legibility of the visualizations.

Adaptive sampling. As there is no prior information available about $\mathcal{P}$, providing an optimal sampling strategy is not trivial. A regular grid sampling is insufficient 


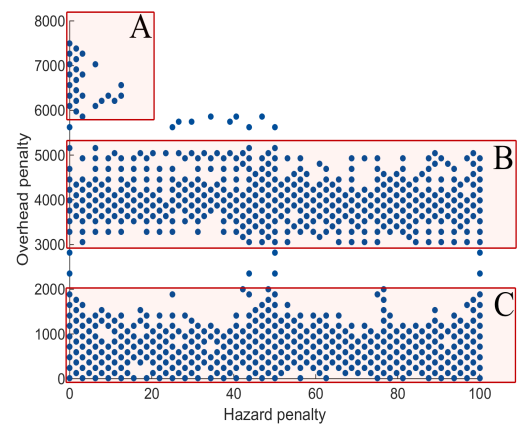

(a) The hazard-overhead subspace of the parameter search space $\mathcal{P}$ shows three path classes.

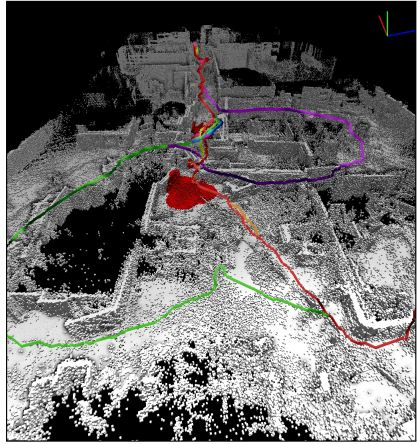

(b) The three path classes clearly show up as distinct when inspected.

Figure 3.17: During the adaptive sampling, it was found that paths cluster in the multi-dimensional space, which correspond to individual path clusters.

as plausible boundary values for each dimension are not known, thus incurring the risk of oversampling or undersampling the path space. In order to sample $\mathcal{P}$ more efficiently, the system utilizes a binary space partitioning such that new samples are only generated in those regions of $\mathcal{P}$ that have the highest potential of providing a new class of path. This assumes a continuity of paths inside of $\mathcal{P}$, meaning that $\mathrm{A}^{\star}(\mathbf{p})=i$ and $\mathrm{A}^{\star}(\mathbf{q})=i, \mathrm{~A}^{\star}(\mathbf{r})=i \quad \forall \mathbf{p}<\mathbf{r}<\mathbf{q} \in \mathcal{P}$.

Using the previously computed paths, the space $\mathcal{P}$ is divided such that new samples of $\mathcal{P}$ are only created where there is the potential of generating novel paths. All potential samples are classified into either non-recursive or recursive samples. In each iteration, each dimension is bisected and the each recursive sample is tested against the neighboring samples to determine whether a region should be subdivided. The subdivision will continue until all recursive samples return non-novel paths or until the distance between samples is smaller than a predefined threshold $\epsilon$. This threshold is necessary as the subdivision would otherwise converge to the boundary and result in an arbitrary large number of samples. This method only requires the specification of arbitrary initial bounds for the weights and the adaptive sampling method will only perform new computations where necessary. Figure 3.17 shows the subspace of hazard-overhead penalty and shows the three groups of paths that are present when visually inspecting the data.

\subsubsection{Rendering}

There are a number of technical challenges that are involved in providing an interactive rendering of the resampled point cloud together with a representation of 


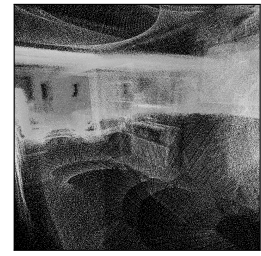

(a) Rendering of the unstructured, unbinned point cloud.

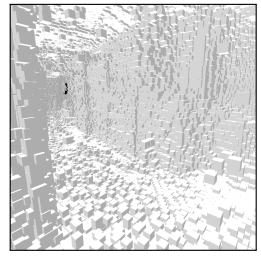

(b) Rendering of the axis-aligned boxes using only Phong shading.

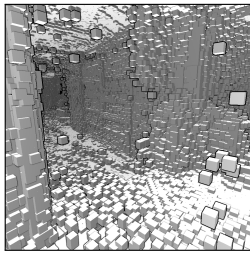

(c) Full voxelized rendering with contours and depth enhancements.

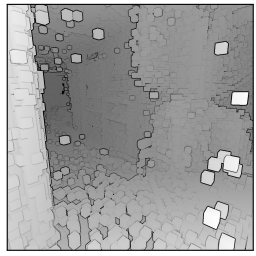

(d) Depth-image rendering that emphasizes the structure of the corridor.

Figure 3.18: Comparisons of the different rendering methods. A brute-force rendering of the original point cloud (a) is memory intensive and does not provide sufficient depth cues. Using the binned point cloud and only applying Phong shading (b) is insufficient to detect corners and judge distance. Using a contour rendering and depth darkening (c) it is possible to detect the structure of the corridor. Using a different color mapping that emphasizes the depth information (d) provides additional information to the user.

the path classes and their analysis tools in the same view. The following paragraphs cover the majority of challenges that had to be overcome.

Point cloud visualization. The results of the point cloud binning is a new, regular spaced point cloud where the center of each voxel is represented by a single 3D position and a global value for the voxel size. This new point cloud is rendered by creating an axis-aligned box for each point. In order to deal with occluding structures, for example roofs, the IC can interactively modify clipping planes that remove parts of the point cloud. Figure 3.18 shows different rendering stages and options that are applied to the visualization; to increase the spatial awareness, we apply a Phong shading based on the face of the cube using a camera-fixed setup of multiple light sources [90] and decided to employ two image-space enhancement methods. The first method is a contour-enhancement that increases local contrast in areas of high depth changes [68]. This is a post-processing step that uses an unsharp masking of the rendering's depth buffer and then uses the result to visually enhance areas of the image with a high depth value gradient. This allows the IC to intuitively gain a better understanding of the scene by emphasizing voxel boundaries. The second method that is also a image-based post-processing is a depth-based attenuation in which every voxels' brightness depend on their distance from the camera, thus providing an distance queue for the IC. As an optional method, a simulated depth image, resembling the output of range imaging cameras already familiar to the IC, is available.

Projective Texturing. An additional method to increase immersion, the system uses a projective texturing to provide access to images from robots and provide more detailed information about the building [39, 105]. Provided with the robot's 


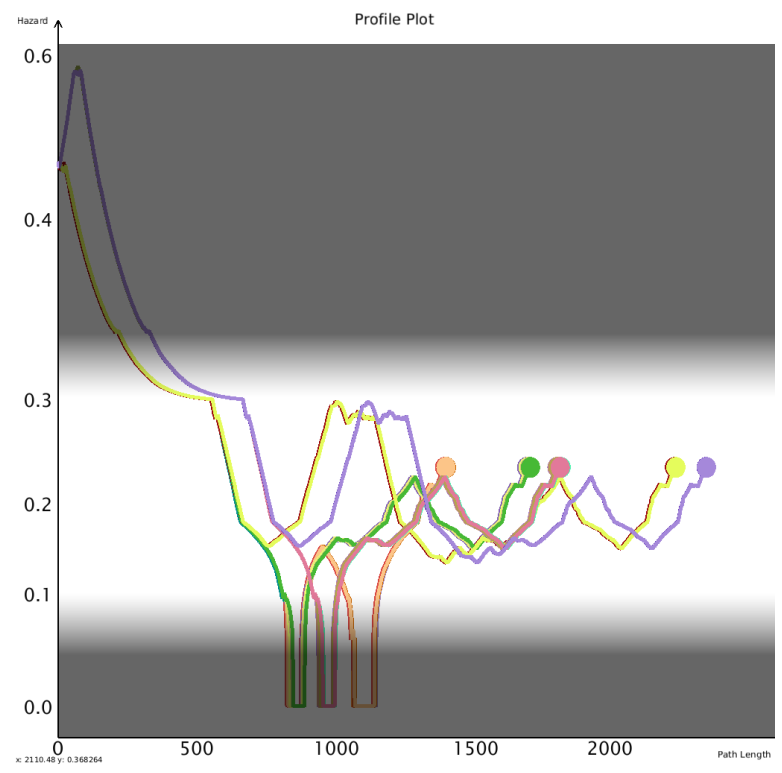

Figure 3.19: The Profile Plot component in the system shows the evolution of a user-selectable parameter over the length of all path classes and makes it possible to compare all available paths in a single view.

orientation and location, information that is readily available, the images or videos are projected onto the voxels [133]. This method enables the optional inclusion of details where the IC requires them, without overloading the user with information.

Bump Mapping. Even though it is possible to represent the occupancy values, which provide feedback about the data reliability, using color information, we found that the color information was used to display the location of hazardous environments instead. Therefore, we use bump mapping that modifies the face normals based on a structured noise pattern with periodic boundary conditions. The displacement depends directly on the occupancy values. Due to the operation of the drones, the occupancy values consist mostly of radial patterns with decreasing occupancy towards the outside, thus leading to an automatic visual fading.

Access path visualization. Each class of paths is represented in the 3D rendering with a fixed color and possesses a vertical offset such that it is rendered on top of the voxel grid, rather passing through the center points. The paths are rendered using Catmull-Rom spline interpolation in order provide a smooth path visualization and not capture the user's attention with jagged edges [26]. Furthermore, if multiple paths pass though the same set of voxels in a row, they are offset horizontally to avoid cluttering and occlusion. In addition to fixed colors 
for each path, the IC can select different coloring schemes to inspect attributes of the path that change along its length. The color values mapped to the path can use any of the derived attribute and thus, for example, show the distance to the nearest hazardous environment for each point along the path or show how much space is available for a responder. The IC can use selected paths for a virtual walk-through to inspect whether a path is feasible based on their own experience. For these walk-throughs, the camera can be steered by the IC directly or it can follow a selected path automatically.

Immersive Environments. The system also provides the IC with the option of rendering the point cloud in an immersive environment, such as multi-pipeline display systems like planetariums or powerwalls, head-mounted displays, or fisheye projections. This technique allows the IC to inspect the rendering using a headmounted display on-sight, which increases the users performance in search tasks [89]. We utilized this method to show a stereoscopic movie of two rescue scenarios to approximately 100 researchers at an international conference on rescue robotics.

Profile Plot Remapping. One of the analysis views employed in the system is a modified line plot that enables the user to inspect a single value along the length of all path classes. In order to make it easier for the user to compare paths, the ordinate axis value range has been modified to include a sub-linear, a linear, and a super-linear part. The cut-off values are chosen by the user to highlight different value ranges. Figure 3.19 shows the Hazard Distance mapped to the ordinate axis. The overlay's transparency shows the amount of performed remapping.

\subsubsection{System}

The entire system consists of four views, the Rendering View, Profile Plot, Parallel Coordinates Plot, and the Scatterplot Matrix, which are organized in a single application (see Figure 3.20). The Rendering View provides the user with an interactive view of the point cloud data in which they can select paths, annotate voxels, and inspect the building in the 3D environment. The other three views support the IC with the analysis of the available path classes. The rendering view (top left) provides the user with a global overview of the structure and the path classes that traverse it. The user selects paths in this view, which are then highlighted in all views. The profile plot shows how the parameter selected by the user changes over the entire length of all path classes. This view can be easily used by the IC to filter paths by their maximum or minimum values and also see overall trends. The parallel coordinates plot shows the derived attributes for all path classes at the same time in order to provide the user with an overview of potential correlation between parameters. Finally, the scatter plot matrix shows the combination of all derived parameters in case it is needed for the IC. 

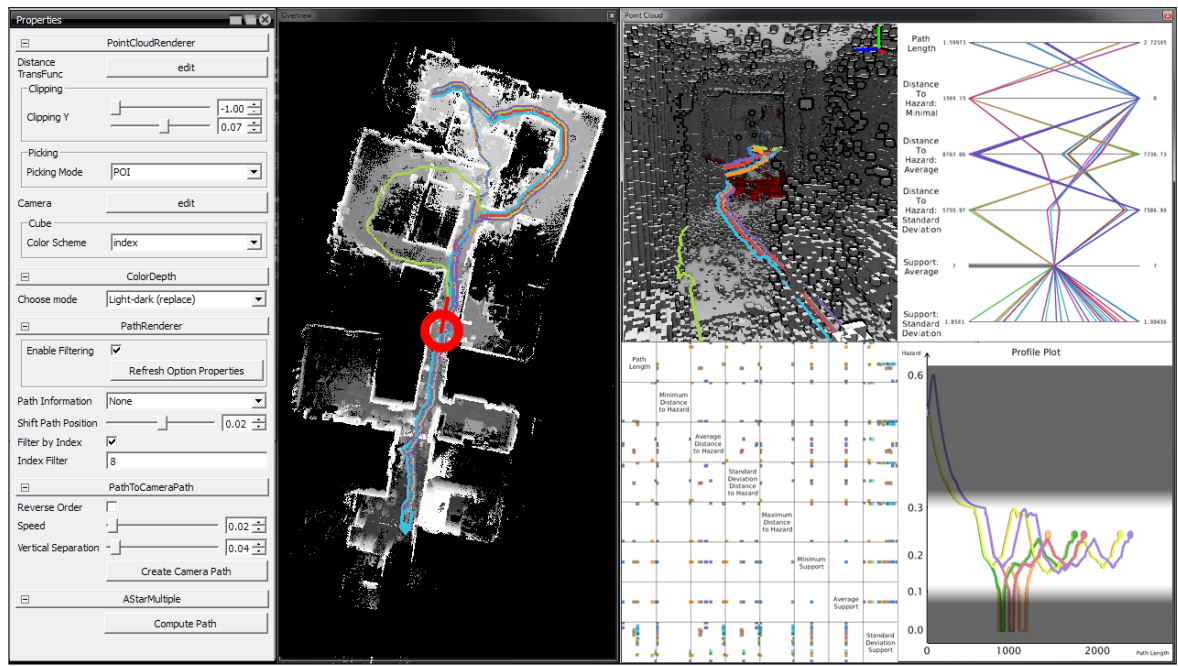

Figure 3.20: The overview of the system consists of four views: the rendering view (top left), the profile plot (bottom right), the parallel coordinates plot (top right), and the scatter plot matrix (bottom left).

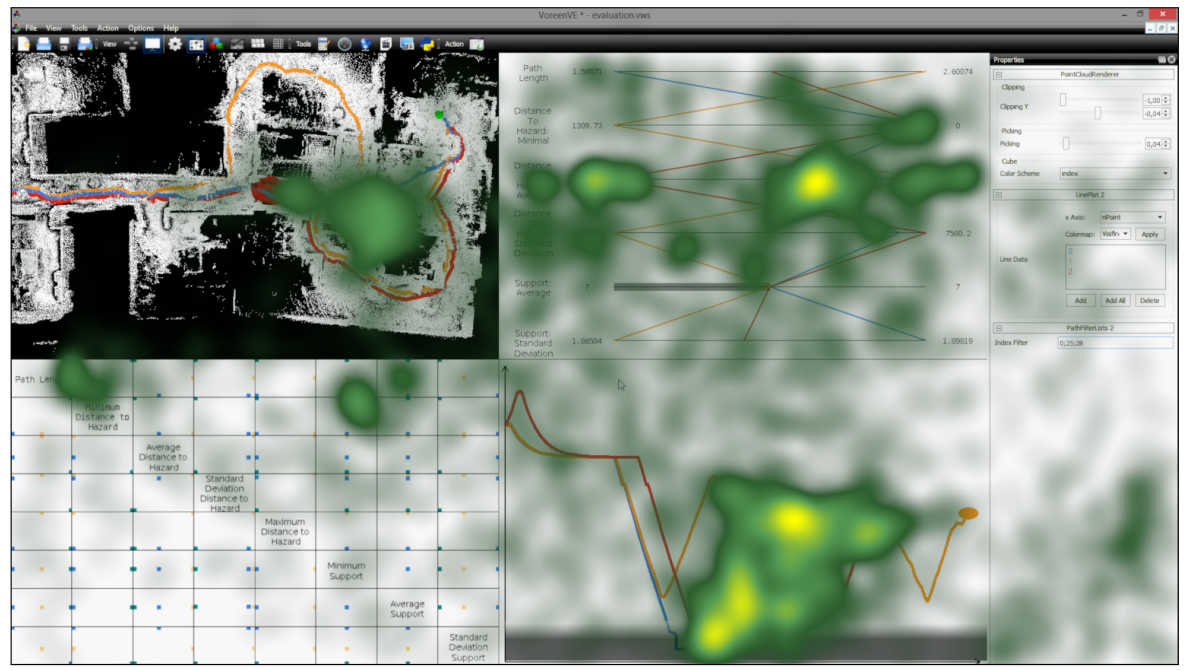

Figure 3.21: A heatmap captured during the second user evaluation that shows the amount of gaze points of one expert while analying a use case over the course of about 15 minutes. 


\subsubsection{Evaluation}

The usability and usefulness of the system was tested using two evaluations. The first evaluation was performed early in the system's development and consisted of an online questionnaire completed by nine international urban search \& rescue experts, composing a mix of emergency responders, researchers, and a consultant for a technical relief agency. During this evaluation, the experts inspected images and videos of the system before answering questions and providing feedback. The results of the seven experts that completed the evaluation are reported in detail in Paper C. The second evaluation was performed with four experts from the Swedish Civil Contingencies Agency and involved interactive sessions with the system which included a think-aloud usage supplemented by an eye-tracking study (see Figure 3.21). During the 45 minutes of the evaluation, the experts were asked to familiarize themselves with the system and inspect one of the prepared cases and make decisions about potential paths for responders. This evaluation was performed using the Think-aloud protocol [59], which was modified following the suggestions of Ericsson to focus on mentioning actions, rather than internal though processes [37]. The results of all evaluations are reported in Paper E.

\subsection{Space Weather Visualization}

The work leading to Paper F was performed in close collaboration with the scientists and space weather forecasters at the Community Coordinated Modeling Center (CCMC) at NASA Goddard Space Flight Center that started two years prior $[14,113]$ and is concerned with the verification of space weather simulations.

\subsubsection{Domain and Scientific Problems}

The CCMC generates time-varying ensemble simulations of the plasma conditions in the solar system in order to further the study of space weather, which describe the Sun's effects on Earth, spacecraft, planetary bodies, and human society. The Sun is constantly ejecting charged particles into the solar system and parameters of this plasma, such as density, velocity, and its interaction with magnetic fields and planetary bodies, have a huge effect humans and our technological devices.

One type of increased activity, called coronal mass ejections (CMEs), can have devastating effects on human technology as; 1 . fast-moving charged particles can destroy satellite electronics and pose danger to astronauts travelling outside Earth's protective magnetic field; 2. the thermal expansion of Earth's atmosphere increases drag and thus reduce the lifetime of operational satellites [51]. An example is Telstar 401, a satellite that was rendered inoperable in 1997 due to a geomagnetic storm [103]; 3. high currents can be induced on the surface by Earth's rapidly changing magnetic fields as it reacts to these particles which can 

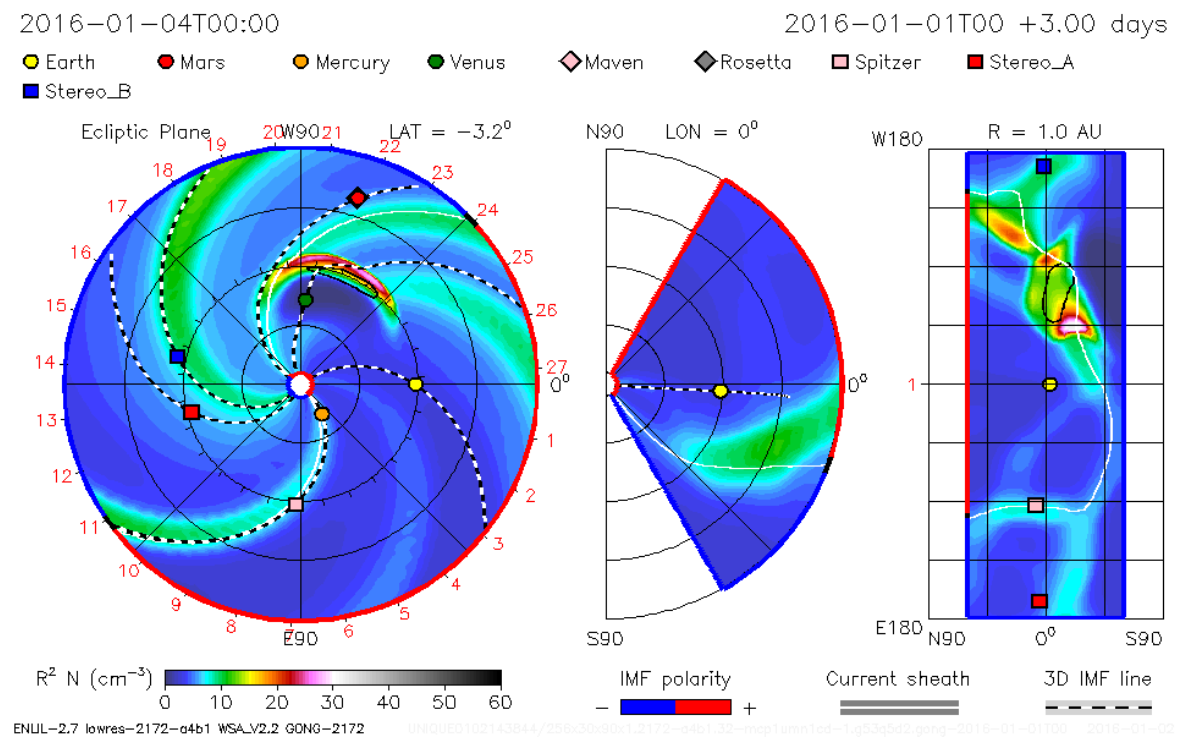

Figure 3.22: An ENLIL simulation time step with an active coronal mass ejection. Previously, the volumetric data was sliced along the ecliptic plane (left), the north-sound plane passing through Earth (middle), and a plane of constant radius (right). These slices make it difficult to analyze the three dimensional structure of the CME.

damage infrastructure. An example of this is the induction of currents in long metal structures on the surface of Earth that can deteriorate, for example, railroads or pipelines [91]. The most destructive, however, is the presence of induced currents in power lines that are directly connected to transformers that can be overloaded and destroyed by the excess energy. In 2003, Llyod's predicted that if the strongest CME ever recorded, the Carrington Event from 1859, were to occur again, the damages to the global economy would be around $\$ 2$ trillion and could lead to power outages of up to 2 years until destroyed transformers are replaced [75]. If operators are informed of CMEs, these scenarios can be mitigated by disabling electrical hardware and astronauts seeking shelter in protected areas. Therefore, it is of paramount importance to provide reliable prediction for these solar events.

Therefore, time-varying simulations are used to predict the impact of the Sun's activity on Earth. The strength of a CME's interaction with Earth's magnetic field is measured by its geoeffectivity and depends, among others, on its speed and the angle between the CME's and Earth's magnetic fields, which is a separate simulation parameter. In the simulations performed at the CCMC, three angles are simulated, $90^{\circ}, 135^{\circ}$, and $180^{\circ}$. 


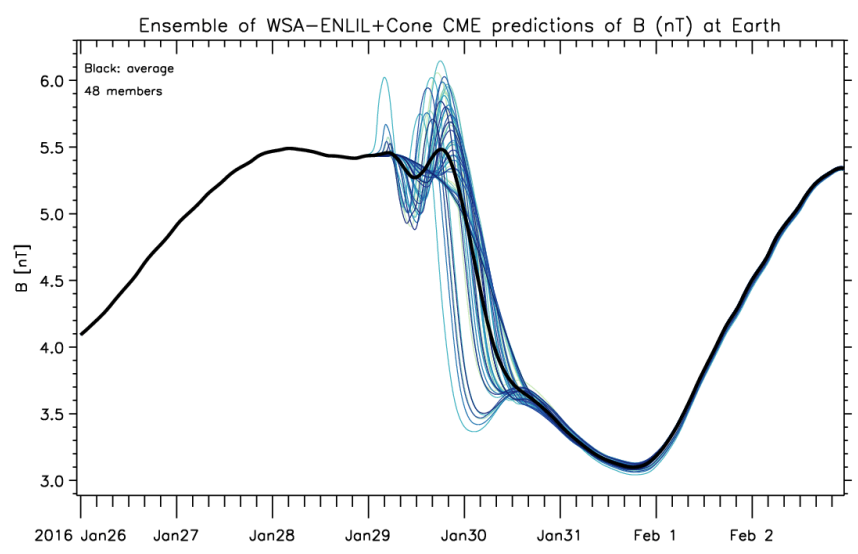

Figure 3.23: The method of presenting the time-varying ensemble results makes comparative analysis difficult as the scientists have to manually compare a set of line plots in order to reach a conclusion.

Currently, most CME predictions are created using a time-varying magnetohydrodynamic (MHD) volumetric simulation called ENLIL in which the input parameters are derived from real-time sun-observing satellite imagery [76]. These simulations create an ambient background flow of charged particles from the Sun and inject a CME event (the model assumes a symmetric cone) that is constrained by the available satellite imagery. The free parameters are determined by the human operators by inspecting the satellite images and measuring the movement of the CME in the available images. The free parameters that are chosen are the location (longitude and latitude), the speed, and the opening angle. Naturally, manual segmentations are not necessarily accurate and can lead to prediction errors that are independent from the accuracy of the simulation model itself. This variation is offset by using ensemble simulations to vary the parameters and perform a simulation for each parameter combination. It is then possible to analyze the predictions and compare these to real-world measurements and enable the scientists to gain deeper insight into potential errors in the simulation models that are independent from the human inputs that were used to create them.

\subsubsection{Application Requirements}

The work in Paper F was performed in order to provide the scientists with better tools to analyze the ensemble simulations of past CME events in detail in order to inform the development of the simulation models and increase the accuracy of their predictive use. Previously, this analysis was done using seperate tools (see Figures 3.22 and 3.23) that prevented the scientists from easily determining the 
root causes of simulation errors as multiple, unconnected systems had to be employed. The goal for this application is to create a single system that combines all information about the ensemble simulations and provides the domain experts with the tools to easily determine the quality of the ensemble simulations and inspect their volumetric simulations in order to view the 3D structure of the CME.

This approach is based on having access to ground-truth measurements of the arrival time, speed, and geoeffectivity recorded on or close to Earth as well as being able to derive values from the simulations and compare these on different granularities. The predictive strength of the simulation depends on the difference between the simulated quantity and the ground truth. The arrival time and geoeffectivity are only known for a single measurement, but the CME is visible in the optical telescopes on-board the observing spacecraft, making it possible to compare the velocity of the CME at multiple points during transit.

This leads to a series of challenges that the system presented in Paper F addresses; 1. The system must present the user with the ability to quickly inspect the relationship between simulation input parameters and the prediction accuracy of each ensemble member. This requires an intuitive visual representation of the $4 \mathrm{D}$ input parameter space; 2 . The user must be capable to inspect the evolution of individual ensemble members over time. This particularly requires the comparison of reconstructed velocity information with the simulated velocity over a large number of time steps and taking into account the different time resolutions of available data; 3. For single time steps of individual ensemble members, it must be possible for the user to view a representation of the volumetric data in its correct relation to the satellites, the Sun, and Earth, making it necessary to accurately position astronomical positions with respect to the simulation data and be able to render transparent geometries combined with rendering multiple volumetric datasets.

\subsubsection{Ensemble Glyph Mapping}

A design requirement for the system was the ability to easily inspect potential correlations between the input parameters and the quality of the simulation results, which previous systems did not support. For each ensemble member, there are four input dimensions, the longitude, latitude, velocity, and opening angle, and four quality values, each differences between the predicted and measured values, arrival time, and geoeffectivity for three clock angles $90^{\circ}, 135^{\circ}$, and $180^{\circ}$. Figure 3.24 shows the 2D glyph-based projections that were designed. For all subviews, the size of the glyphs correlates with the opening angle of the ensemble member, a bigger opening angle resulting in a larger glyph. Some ensembles also contain an additional ensemble run using the average parameter set which is denoted with a + symbol. If the user selects an ensemble member, it is highlighted in all three views. The main view (top left) shows the ensemble members mapped into the longitude/latitude space. The mapping is performed linearly using the 


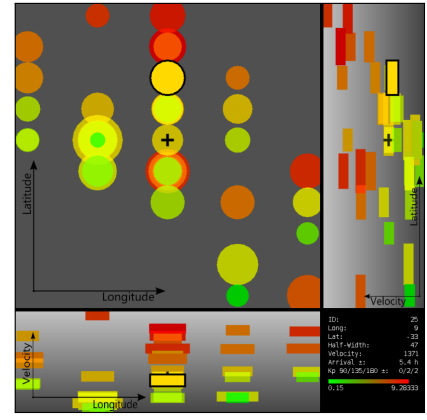

(a) Arrival time prediction

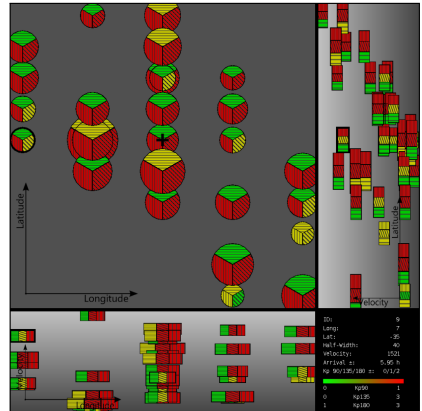

(b) Geoeffectivity prediction

Figure 3.24: These views show the two modes of the glpyh-based ensemble member representation. The free parameters longitude, latitude, velocity (location), and opening angle (size) are mapped in three views and the accuracy of arrival time (a) and three geoeffectivities (b) are shown color-coded.

minimum/maximum values of all ensemble members. The glyphs of ensembles with larger opening angles are rendered first, such that smaller angles overpaint and are still visible if multiple simulations were performed at the same location. The other two views provide replace one of the longitude or latitude axes with the velocity of the CME. The views are arranged such that the remaining quantity acts as a projection of the main view with the velocity being the distinguishing factor. Finally, an additional window provides numerical information about the currently selected ensemble member.

The entire view can be switched to show either the accuracy in CME arrival time or its geoeffectivity. In both cases, color represents how well the simulation agrees with the measured data. This value is normalized to 0 and the maximum error for the entire ensemble and shown using a green-red color scale. For the arrival time, the entire glyph is colored uniformly (see Figure 3.24(a)). There are three predictions for the geoeffectivity and each glyph is split into three segments. The magnetic angle is represented by the angle of the textured lines in that segment.

\subsubsection{Optical Flow Analysis}

It was necessary to reconstruct the CME's velocity from the available satellite images. An optical flow algorithm computes a vector field describing the movement of objects between two input images and thus describes how individual features move between subsequent frames. While this has been shown to work with solid objects, Colaninno et al. also found that it was feasible to determine the tangential velocity of CME shock fronts using optical flow analysis [27]. In this case, subsequent images from the major sun-observing instruments (STEREO's Cor2, HI 1, HI 2, 


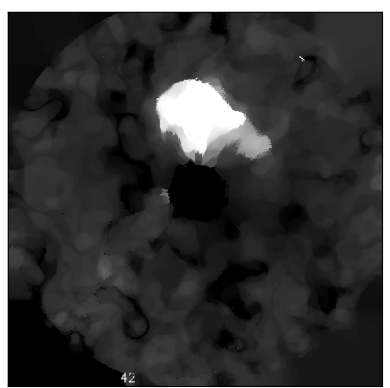

(a) Length of the optical flow derived velocity vector for each pixel in the Cor2 image.

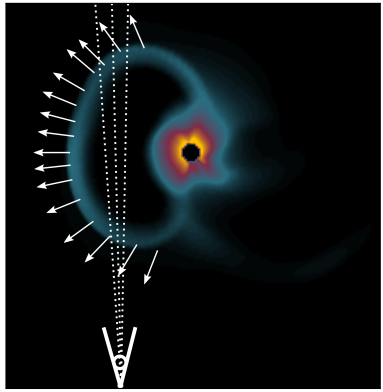

(b) Illustration of the projection of the volumetric velocity field along a view ray.

Figure 3.25: The construction of a velocity timeline for each ensemble member includes extracting the velocity from subsequent pairs of satellite images for each instrument (a) and retrieving the simulated velocity for each position from the volume (b).

and SOHO's LASCO C3) are used to compute the optical flow for each pixel using the algorithm presented by Sun et al. [111]. Figure 3.25(a) shows the result of this algorithm on the Cor2 instrument. In order to retrieve a single, representative velocity for the CME, each velocity vector is first replaced by its length. Then, the bottom $75 \%$ of data values are discarded in order to remove the slow-moving background data and, finally, a representative velocity value for the entire image is computed by averaging the remaining $25 \%$ of values.

In order to be able to retrieve a comparable velocity from the volumetric simulations, it is necessary to reproject the velocity values of the simulation for each instrument. For this, the simulation was rendered using volumetric raycasting with the camera placed at the satellite's location with the instrument's field-of-view settings. For each pixel, the simulation's velocity is sampled and a running average of velocities is constructed (see Figure 3.25(b)). As the optical flow algorithm can only detect tangential velocity, the simulated velocity first has to be projected into the tangential plane before adding. Then, similar to the optical flow, the final velocity is the average of the top $25 \%$ of the velocity vector's lengths.

\subsubsection{Rendering}

The rendering in this application mixes opaque geometry of solar system bodies, transparent satellite images, and a multivolume raycasting. One of the important technical aspect of this is the accurate positioning of all objects involved in the scene have to be accurately positioned. For the Earth, the Sun, and the involved satellites (STEREO A, STEREO B, and SOHO), we use of the SPICE library provided by NASA's Navigation and Ancillary Information Facility [2]. 


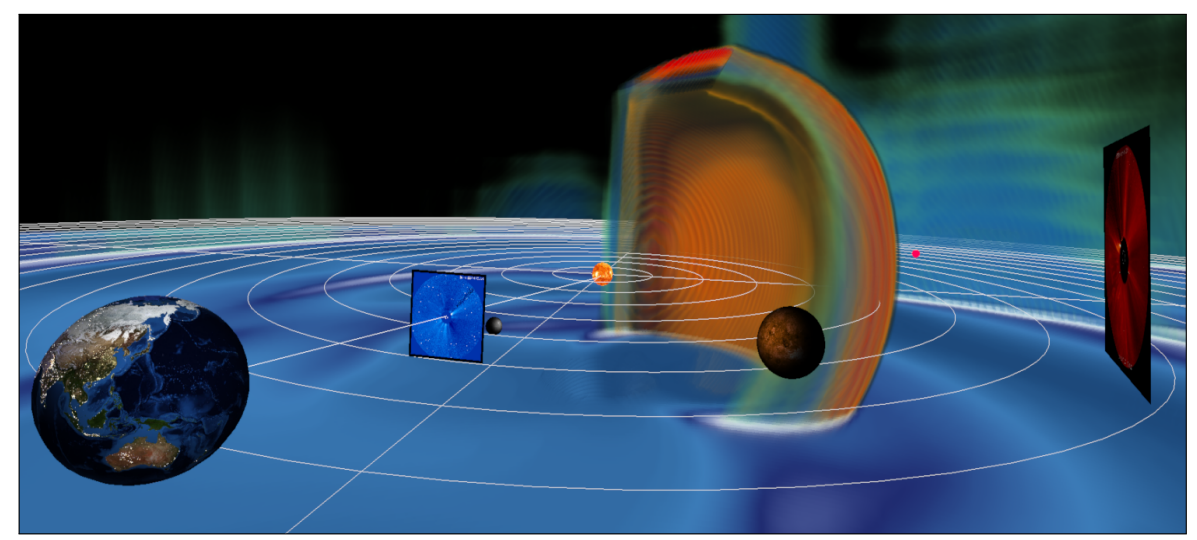

Figure 3.26: The rendering of this scene requires the support of accurate locations as well as the ability to render transparent geometry together with the volume data.

The volumetric simulation, on the other hand, is provided in the HEEQ coordinate system, which uses the Sun's rotation axis and the vector between Sun and Earth at an epoch and constructs a right-handed coordinate system from this. With this information it is possible to compute a model matrix that orients the volume correctly in relation to the other scene elements.

The satellite images are rendered along the view of each instrument. The user can change the distance of the image plane from the satellite and thus its absolute size in the solar system (see Figure 3.27). The image planes can be rendered semi-transparently, which enables the user to place the camera at the satellite's position and compare the image with the simulations (see Figure 3.28).

For the volumetric rendering, we render two parameters that are included in the simulation. During the development of this application, the scientists found that it was beneficial to perform each simulation once without a CME to produce the ambient background conditions, and once with the injected CME. The volume rendering uses the difference between the two values, thus showing the effects of the CME while suppressing the background conditions.

The datasets are natively produced on a spherical grid which is stored as a regular structured 3D cube

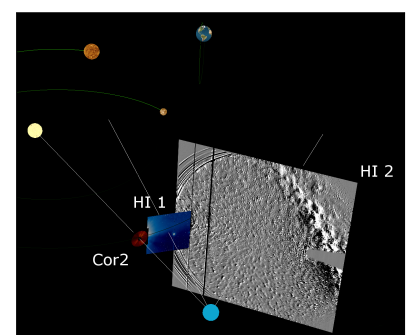

Figure 3.27: Orientation of the various image planes. on the GPU using $r, \phi$, and $\theta$ as the orthogonal coordinate axis, similar to the approach by Balabanian et al. [4]. The ray marching is performed in Cartesian coordinates, but for each sample location, these coordinates are converted into spherical coordinates before sampling the volume. This has a number of beneficial 


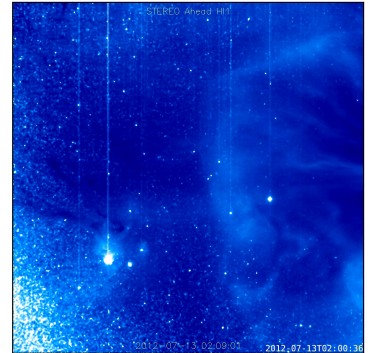

(a) The satellite image of STEREO's HI 1.

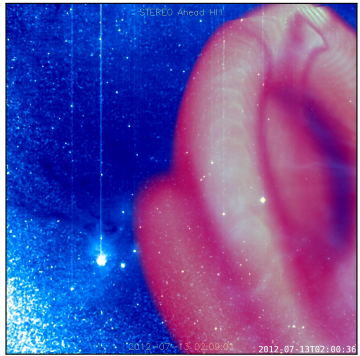

(b) Combined satellite image and simulation

Figure 3.28: The ability to control the image transparency is important to allow the user to compare the predictions of the simulation to the acquired ground-truth images.

characteristics. The trilinear interpolation that is performed on sampling in spherical coordinates produces the same results as a spherical linear interpolation, which is more beneficial for datasets with spherical symmetry. In addition, the non-uniform data distribution in Cartesian coordinates presents a basis for adaptive sampling, with a higher data density close to the Sun when the CME is physically smaller. The stepsize during the raymarching is modified by using the radial distance of each sample to the center of the volume. Lastly, the volume rendering employs object-order empty-space skipping by utilizing a tessellated sphere as the bounding box and we found the tessellation error to be negligible. In order to support the combination of transparent geometry and volumetric rendering, improvements to the work of A-buffer implementations was developed that support multiple volumes and multiple geometries in the same scene [63].

\subsubsection{System}

The application consists of three separate views, the Ensemble View, the Timeline View, and the Spatial View (see Figure 3.29). The Ensemble View uses the glyphbased representation of ensemble members (see Section 3.4.3) and provides the user with an immediate view of the general trend of simulation accuracy. The user can select individual ensemble members, which can then be inspected in the next view. The Timeline View shows the velocities for each instrument for each spacecraft extracted using the Optical Flow algorithm over time. The user can inspect the extracted velocities at each time using the mouse and when selecting a time step, its datasets are loaded into the last view. The Spatial View makes use of the rendering algorithms described earlier in order to present a detailed view of the solar system's state at the selected time for the selected ensemble member. Naturally, the locations and images of the satellites will be the same for all ensemble members, but the simulation results will be different. 

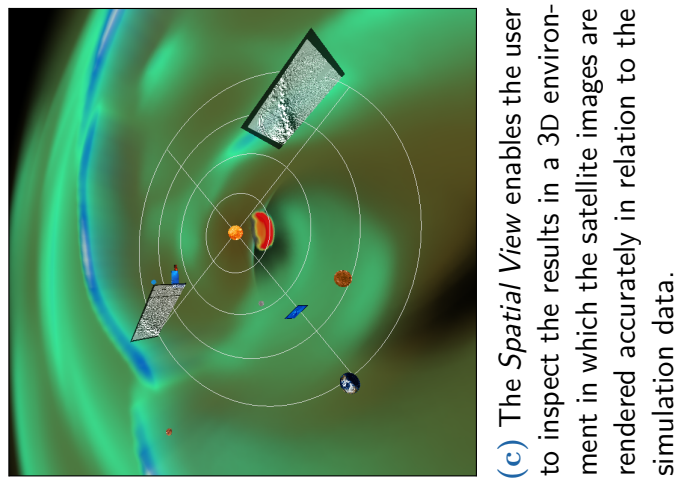

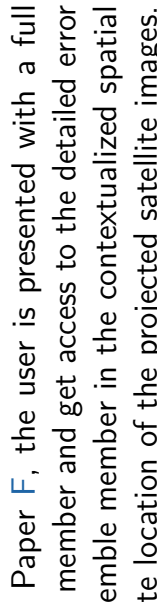

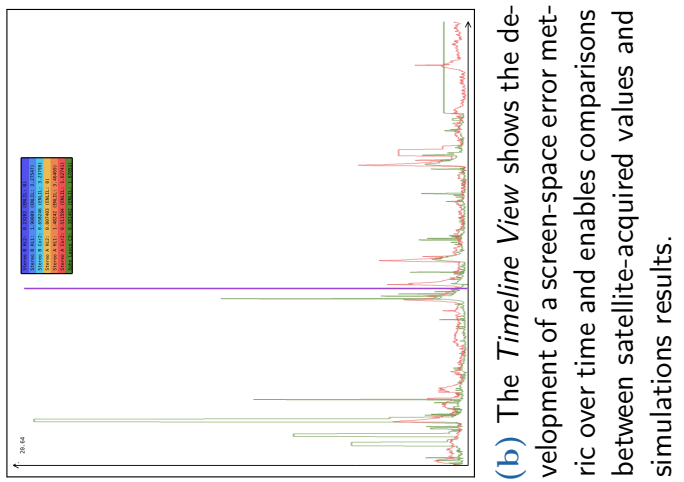

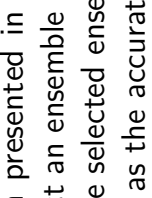

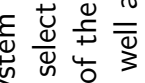
ते

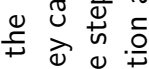

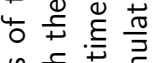
Uै

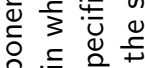
है $\frac{0}{\pi}$ क ปิ

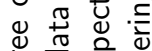

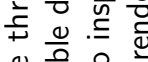

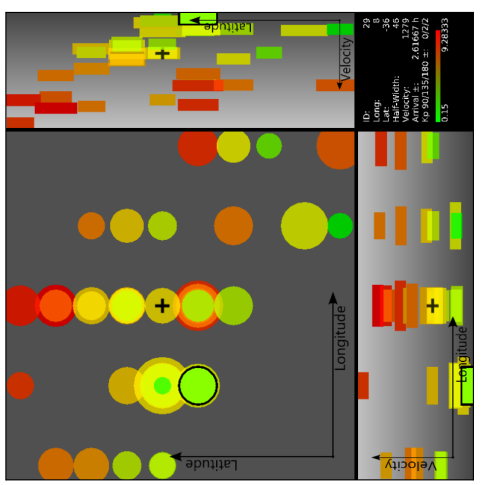

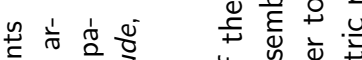

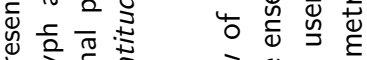

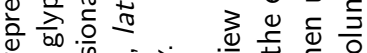

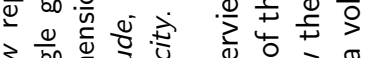

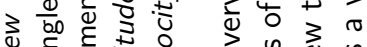

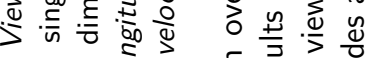
थ है حㄴㄴ है 岀

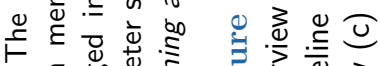
త) 


\subsection{Astronomical Visualization}

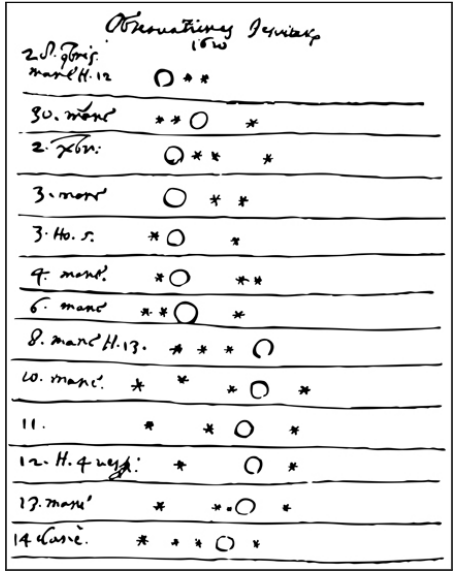

Figure 3.30: The original drawings by Galileo Galilei of the movements of Jupiter's main moons which enabled the discovery that these are objects orbiting Jupiter and thus informed the discovery that the Earth orbits the Sun.
Visualization has been employed in the field of astronomy and astrophysics since its beginning. An early example is the usage of constellations as visual abstractions to map the night's sky impacting human culture. A more modern example are the drawings by Galileo Galilei that, for the first time, systematically documented the movements of Jupiter's moons. Visualizing these movements enabled Galileo to discover the heliocentric structure of the solar system (see Figure 3.30) [41].

However, especially since the introduction of computers into the visualization work, these two fields have expanded rapidly in concert, and these days visualization systems are used in a large number of applications that span the entire spectrum from initial knowledge discovery, such as a scientist analyzing simulation results, all the way to public dissemination, such as Jim Blinn's 1978 animations of the Voyager spacecraft.

The following contributions follow the same directions as these last two historical examples: the use of a single visualization application as a support for hypothesis generation and its use to communicate engineering or scientific endeavors to the general public. This was enabled through the development the software tool OpenSpace.

\subsubsection{OpenSpace}

The OpenSpace project was started in 2014 with the goal of creating an open-source software that leverages synergies between the three categories of visualization in the context of astronomical visualization [19]. These aspects are the availability of the tool as a research tool for domain scientists for hypothesis generation and validation, its usage as a research platform for development of novel visualization research, and as a means to support the public dissemination of scientific discoveries. By combining all three of these aspects into a single software platform, it is possible to shorten the deployment cycle of new visualization methods into the hands of domain scientists and the general public. At the same time, it provides the domain scientists with an avenue to quickly disseminate their research findings to the general public and provide their data to visualization researchers at the 
same time. An additional benefit of providing all of these developments in a single platform is the ability to display individual discoveries in their proper context, for example by showing the articulations of a surface rover on Mars in its correct surface environment, which was reconstructed from the images of orbiting satellites, or being able to present the movements of a spacecraft in the solar system while providing the image of the Milky Way and correctly aligned stars in the background as a reference point and to increase immersion for the general public.

In order to support this effort, the software targets a variety of display systems, such as home computers, virtual reality headsets, and multi-pipeline display systems like planetarium domes or powerwalls. This flexibility is one of the enabling factors to shorten the deployment cycle between the three pillars of the application as researchers, in most cases, do not have to invest any time to convert their data or algorithms to switch between any of the display modalities.

The rest of this section describes the papers that were published in this ongoing effort. Paper G describes the development of a dynamic scene graph that enables the display of objects across large scales (Section 3.5.2), Paper H describes a system for high-fidelity rendering of planetary surfaces that support up to micrometer resolutions in order to be able to display geospatial discoveries in their correct spatial context (Section 3.5.3), and Paper I presents the visualization of spacecraft operations and image projections using OpenSpace (Section 3.5.4).

\subsubsection{Dynamic Scene Graph}

One major feature of OpenSpace is the ability to display every available dataset in a single common reference frame in their correct context. This necessitates the ability to accurately express the position of objects across enormous scale differences and provide the ability to render these simultaneously. One example of this is the ability to render an Earth-orbiting satellite while also seeing the Cosmic Microwave Background radiation at the edge of the Observable Universe, a scene that covers a scale difference of $10^{26} \mathrm{~m}$. The usefulness of portraying these scale differences for storytelling purposescan be seen in the Powers of Ten animation by Eames and Eames [82].

\section{Scientific Problem}

One intrinsic challenge of handling these vast scale differences is embedded into the traditional way of organizing a scene through a scene graph by constructing a directed, acyclic graph that describes a hierarchy of nodes in which each node's position and orientiation is expressed relative to its parent. During a traversal of the scene graph, the relative positions and orientations for each node and its ancestors are accumulated in order to transform an object into the coordinate system defined by the root node. The camera, on the other hand, is only expressed as a single offset vector and orientation relative to the root node. 


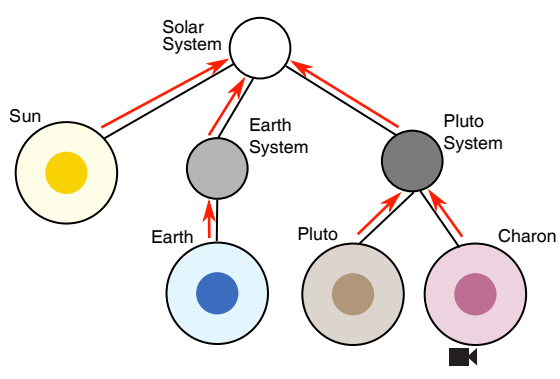

(a) The traditional scene graph evaluation for an exemplary node.

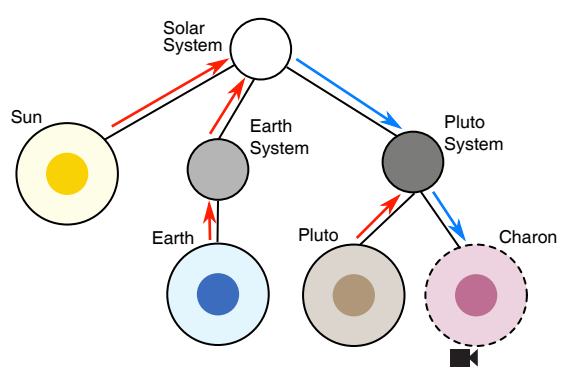

(b) The same node evaluated with the dynamic scene graph.

Figure 3.31: The traditional and the dynamic traversal methods applied to a sample scene graph.

This provides a problem if the camera is pointed at an object that is at a great distance from the coordinate system origin as the construction of the view matrix in this case can lead to catastrophic cancellation [29]. 32 bit floating point numbers standardized in IEEE 854 have a machine epsilon of $2^{-24} \approx 6 \cdot 10^{-8}$, which places an lower limit on the minimum expressible distance between two subsequent numbers. For any 32 bit IEEE 854 floating point number $n$, every number $\left[n-n \cdot 2^{-24}, n+n \cdot 2^{-24}\right] \backslash n$ is ill-defined and will be rounded to the nearest representable value. As an example, the distance from the Sun to the center of the Milky Way (a plausible coordinate system origin) is $8 \mathrm{kpc}$ or $\approx 2.5 \cdot 10^{20} \mathrm{~m}$. One method of defining the size of the solar system is the distance to the heliopause, the area in which the Sun's solar wind is no longer dominant, which provides a size of $120 \mathrm{AU}$ or $\approx 1.8 \cdot 10^{13}$. Given these values and a naïve scene graph implementation, the bounded distance between floating point values at the Solar System would be $2.5 \cdot 10^{20} \mathrm{~m} / 6 \cdot 10^{-8}=1.5 \cdot 10^{13}$, which means that there would only be a single valid 32 bit IEEE 854 floating point number for the entirety of our solar system, which is insufficient for any meaningful visualization.

\section{Algorithm}

Paper G presents a modification to the traversal algorithm employed in standard scene graph methods that enables a much larger range of values to be represented in the same scene. Instead of being expressed relative to the root node of the scene graph, the camera is instead always attached to the closest scene graph node, which is called the attachment node. The traditional traversal is then replaced with a dynamic algorithm that can traverse the scene graph in both directions in order to project every object into the local coordinate system of the attached node (see Figure 3.31). Figure 3.32 shows the result of this in a real-world example of the Voyager spacecraft at a distance of approximately $21.17 \cdot 10^{15} \mathrm{~m}$ from the Earth. 


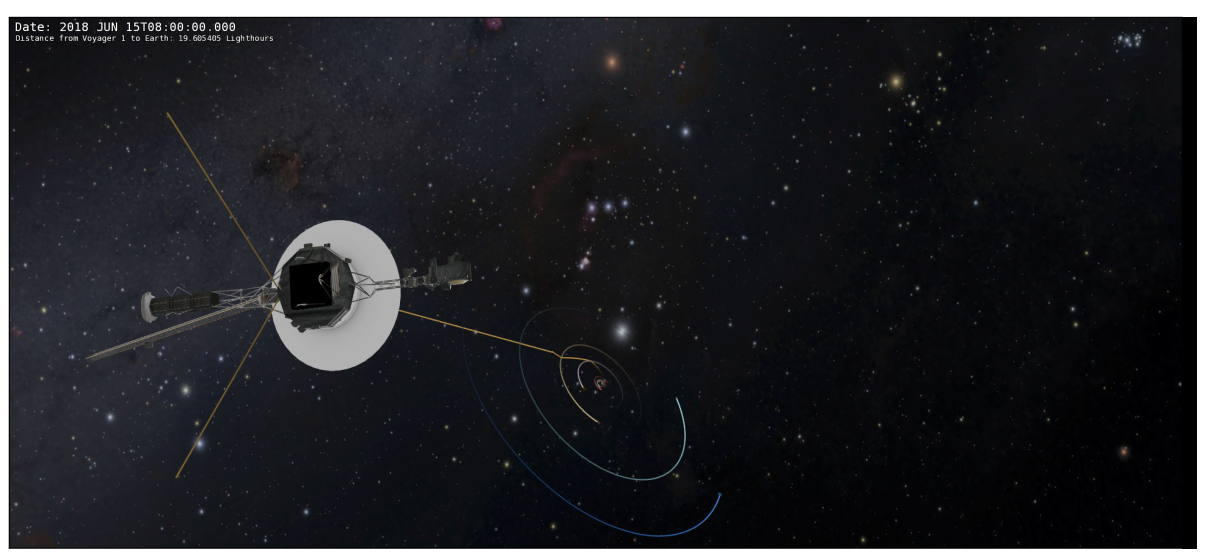

Figure 3.32: A rendering of the Voyager 1 spacecraft in the year 2018 at $21.17 \cdot 10^{15} \mathrm{~m}$ or 19.6 light hours from the Earth.

The only additional limitation on the relative scene graph node transformations is that they can be inverted, which is trivially achievable for all regular transformation matrices.

The dynamic traversal of the scene graph operates by finding the shortest path between every node and the current attached node in the graph. The transformation uses the stored relative transformations for each traversal step towards the leaves, and the inverse of a transformation for each movement towards the root.

Figure 3.31(b) shows an example of these traversals with the blue arrows representing the stored transformations and the red arrows illustrate the inverted transformations. Using the shortest path utilizes only the minimal subgraph and thus avoids any unnecessary large, and potentially catastrophic, transformations to other parts of the scene graph. In the example, rendering the location of Pluto does not require the knowledge of where it is located relative to the Sun, thus removing any catastrophic cancellation that might occur due to that transformation. For the earlier example, rendering anything in the solar system does not require the large distance from the Sun to the Milky Way center and thus avoids the catastrophic cancellation in that instance, too.

An alternative way of implementing the algorithm is using a traditional scene graph traversal, but the scene graph is rearranged every time the attached node changes such that the attached node becomes the root of the scene graph, which includes inverting transformations between nodes, where necessary.

A critical component that the dynamic scene graph requires in addition to traditional scene graph implementations is logic for handling the attachment of the camera. Figure 3.33 shows an example of an attachment node switch when moving from node $\mathrm{N}$ to its parent $\mathrm{M}$ where in infinite precision math, $\mathbf{v}=\mathbf{n}-\mathbf{w}$. This switch 

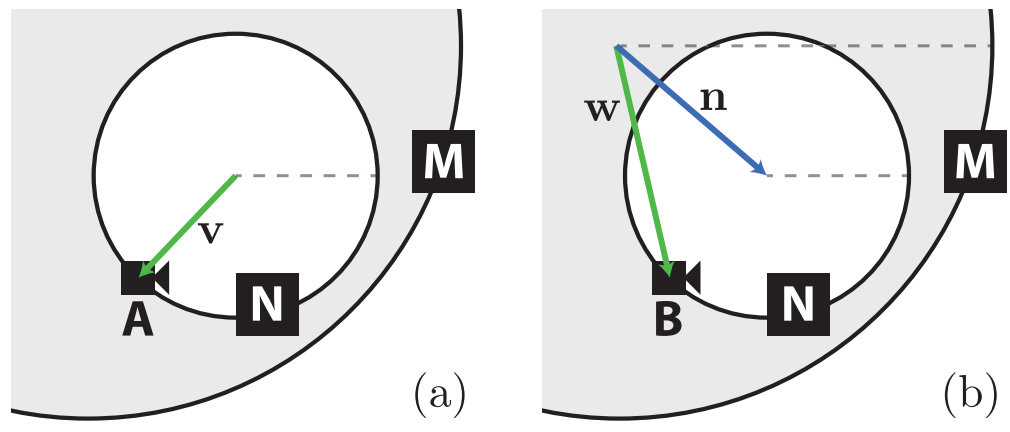

Figure 3.33: The camera's attachment node before and after a switch of nodes.

happens at a distance $d$ from the current attachment node. In IEEE 854 floating point math, this limits the relative transformations between parents as imprecisions here will lead to a camera jitter when switching between attachment nodes if too many details of the focus object are visible at the distance $d-$ if $d$ is large, then the object will only be represented by a single pixel, any jittering of the camera will be negligible. In the case of OpenSpace, the default value for $d$ is $10 \times$ the object's bounding box, which provides reasonable results.

While this mechanism solves the problem of rendering objects that are close to the camera, as the traversal in the scene graph does not involve large numbers, it does not inherently solve the challenge of rendering small objects that are far away as small variations in the object's geometry are still subject to catastrophic cancellation. However, this situation rarely occurs in practice as small, distance objects will generally not be visible with normal field-of-view parameters.

\subsubsection{Planetary Rendering}

A vast amount of information has been gathered not just about Earth, but other planetary bodies in the solar system as well. High-resolution images of terrain features are available for the Earth, the Moon, Mercury, Mars, 67P-ChuryumovGerasimenko, Pluto, and Charon. Aside from showing visually appealing renderings of planets, these images are essential to provide other scientific discoveries in their proper context, for example planning the landing and subsequent movement of rovers on Mars. The rendering system presented in Paper $\mathrm{H}$ was developed to provide this contextualization for all planetary bodies in the solar system by using adaptive, multiresolution data streaming to retrieve image data from online data sources and make it possible to render planetary-wide datasets and contextualized micrometer images simultaneously. 


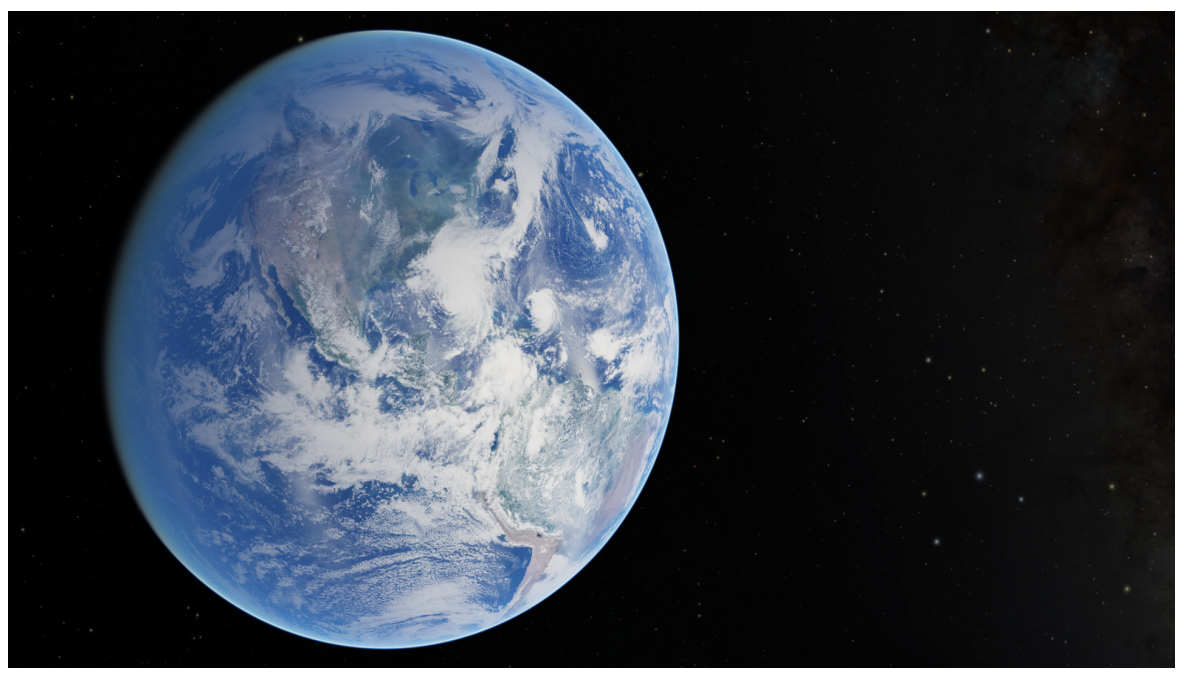

Figure 3.34: A global rendering of the Suomi-NPP's VIIRS instrument. The instruments provides daily, corrected reflectance image of the entire earth at around $400 \mathrm{~m}$ per pixel resolution.

\section{Scientific Problem}

Many institutions provide high-resolution imagery data as free online repositories. NASA's Global Imagery Browse Services provides over 200 image products, many of which are updated at least daily, showing global time-varying aspects of Earth, for example global weather patterns (see Figure 3.34). A second example is NOAA's Science on a Sphere, which is both a visualization modality as well as a dataset curation effort for scientific storytelling [69]. However, a system that enables the public and experts to combine all available repositories interactively for story telling was previously missing. Such a system must enable the display of image data, digital terrain models, and provide current blending information to provide adequate contextualization.

Aside from global time-varying datasets, a second use case is the display of highresolution terrain models used as context for the dissemination of science. Also, no freely available system was present to display global datasets in the same context as submeter resolution terrain models. These datasets are available on the Moon, Earth, and Mars, where they are used for mission planning (see Figure 3.35).

A further limitation of previous systems is the restriction to near-spherical geometries. Some bodies where images are available are, for example, minor moons such as Saturn's moon Prometheus (see Figure 3.36). Handling these more complex triaxial ellipsoid geometries in the same system is desireable. 


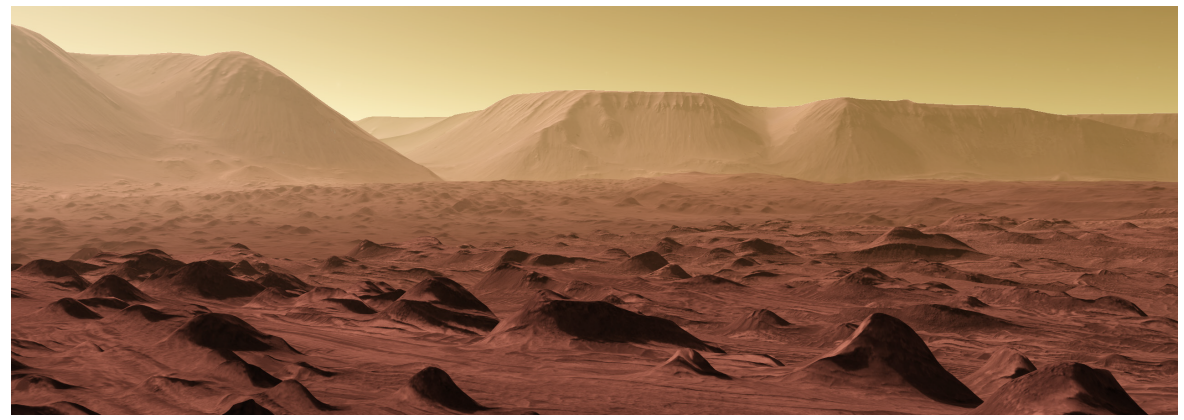

Figure 3.35: High-resolution terrain model on Mars showing structures up to $25 \mathrm{~cm}$ resolution that are derived from the Mars Reconnaissance Orbiter's HiRISE camera using stereoscopic photogrammetry.

\section{Data Access}

All data in the system is provided as 2D images, which can encode a variety of dataset types. The available data types are regular image data, digital terrain models (DTM), or binary masks. Image data can be natively displayed using either RGB or RGBA images; DTMs are gray-scale images that encode the height offsets from a tri-axial reference ellipsoid; and masks are binary images that are used, for example, to restrict the specular highlights to water surfaces. The most common access method for global image sources is an internet-based level of detail protocol called the Web Map Service (WMS) [32, 72]. This protocol provides discrete image tiles, the parametrization of which depends on the protocol variant. The Tiled

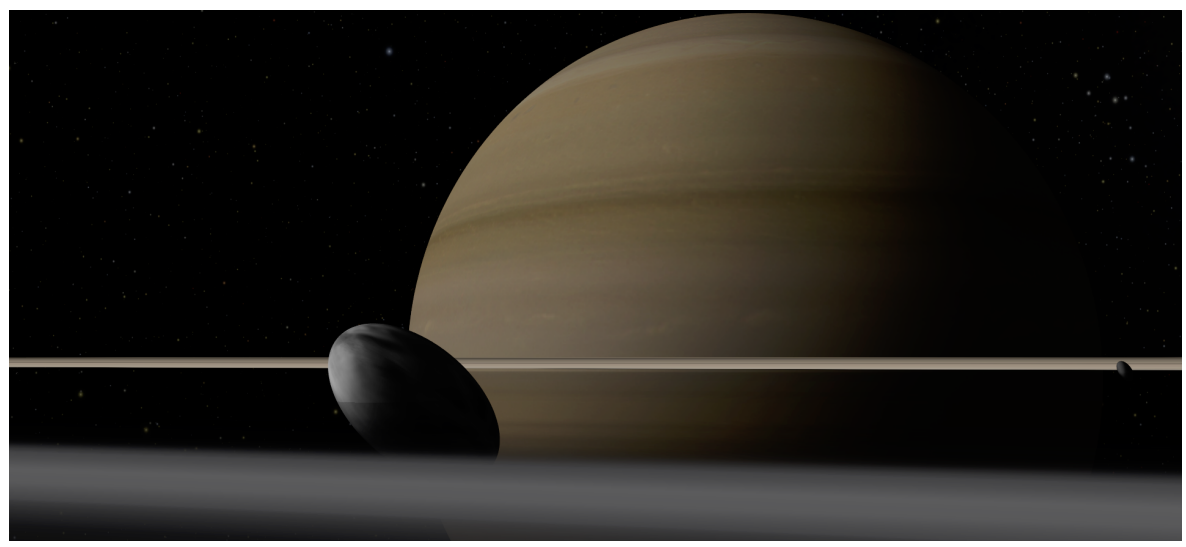

Figure 3.36: A non-spherical terrain model showing Saturn's moon Prometheus in the front and it's companion Atlas on the right. 
Web Map Service (TWMS), for instance, uses a 3D coordinate $\mathbf{c}=(x, y$, level $)$ that describes each discrete tile on a globe. $x$ and $y$ can be constructed from a point's longitude and latitude and the globe's radii, whereas the level depends on the desired granularity of the map.

Local image data is often provided in the form of georeferenced Tagged Image File Format (GeoTIFF) files, which provide embedded longitude and latitude information [95]. The image products of the Lunar Reconnaissance Orbiter (LRO) [122] or the Mars Reconnaissance Orbiter (MRO) [77] are provided to the public in this method. Other providers utilize different image formats, such as PNG files, which are not georeferenced, but show global phenomena instead. Many of these files are provided in a large variety of coordinate systems, or projections.

The Geospatial Data Abstraction Library is a freely available library that provides a layer of abstraction between the usage of these image formats and their concrete implementation [125]. This makes it possible for application to support a variety of data formats without the requirement of implementing a large number of concrete protocols. The library also provides the ability to reproject images between a large number of available map projections. Restricting the rendering system to a single map projection (the equirectangular latitude/longitude projection) increases resilience and performance of the system as reprojected images can be cached locally and thus its performance cost can be amortized.

\section{Rendering}

The rendering system is based on a chunked level-of-detail algorithm presented by Ulrich [118] and Cozzi and Ring [28]. All chunks are organized in a quadtree in which the level of tree determines the extent of the geographic area that is covered by chunks at that level. The tree is traversed using the current camera location and distance which determines whether a node in the quadtree should be subdivided or not. After the evaluation, all leaf nodes of the tree are then rendered using skirted grids (see Figure 3.37). The chunks only represent geometry information and provide texture coordinates. The images that are used for the chunks are organized in a separate tile tree in order to enable dynamic data loading and enable the rendering of geometry even when images of a desired resolution are not yet available. In addition, this separation enables the combination of data sources of different resolution, such that lower resolution textures can be used until a desired level has been requested, if it is available.

The rendering supports a number of different layer types, which determine the way it is used in the rendering step. If multiple layers of a single type are enabled simultaneously, layers are stacked, similar to a layer feature in image editing applications. The Color Layer is interpreted as a, potentially transparent, color texture that is applied to the chunk. A Height Layer's texture information is used to offset the vertices of a chunk in order to generate a high-resolution $2.5 \mathrm{D}$ local 


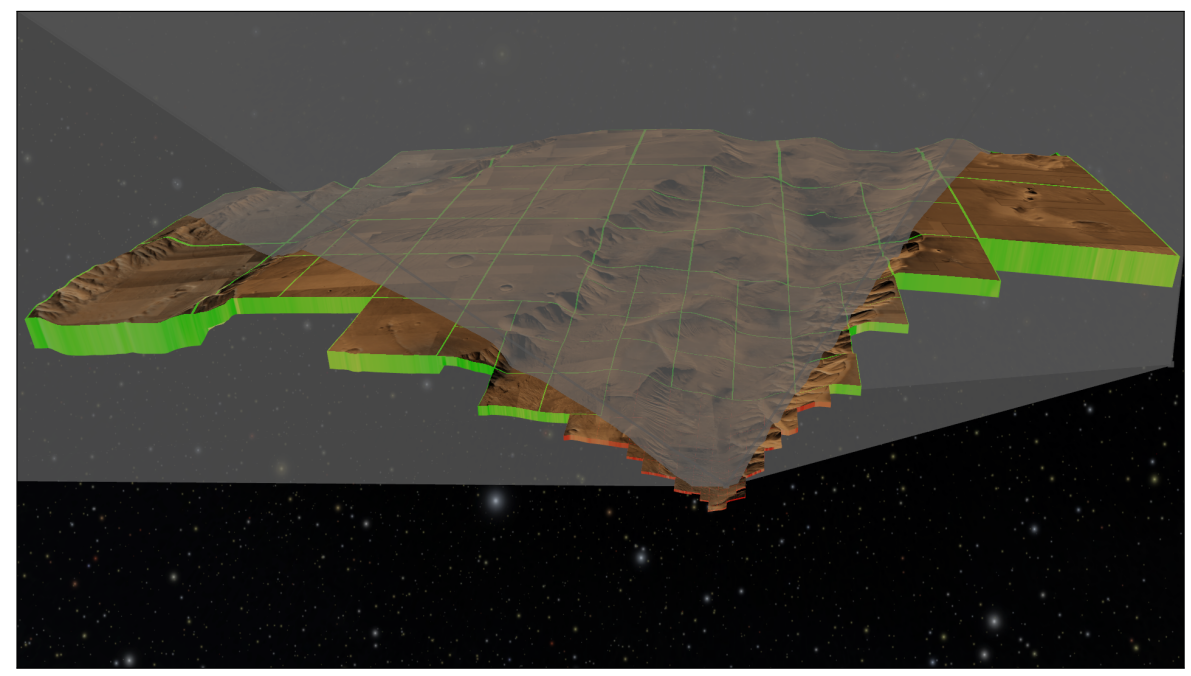

Figure 3.37: The chunked level-of-detail algorithm used in the terrain renderer renders higher resolution images close to the camera and enables the culling based on the view frustum and the horizon.

terrain model. A tile's skirt length is inversely proportional to the tile level and are added to the chunks to prevent holes in the model where adjacent chunks's DTM data is present at different resolutions. The Water Mask is a binary image that masks water on a planetary surface. This information is used in the lighting model to determine the specularity of each location such that only water surfaces are reflective. The Night Layer is similar to the Color Layer as it represents a native image texture. However it is only used on the side of the planet that is facing away from the primary light source. On Earth, this is used to show the night time illumination of the major cities and the surface illumination of the moonlight. Finally, the Overlay Layer is a transparent layer that is rendered on top of other layers and is used for informative overviews, such as country or city names, or reference labels to natural landmarks.

Time-varying datasets are handled by creating a separate tile tree for each time step that is requested and keeping a cache for all image tiles that have been requested, thus conceptually increasing the access coordinates to $\mathbf{c}^{\prime}=(x, y$, level, $t)$. No other change to any of the rendering has to be performed due to the way the image tiles are created and stored separately from the geometry. This holds true for all available image layers types, thus natively enabling time-varying color images, as well as, terrain information.

The evaluation of the chunk tree utilizes two culling steps that remove quadtree nodes if they are not visible in the final rendering. The frustum culling uses the 
camera view frustum and each chunk's bounding box to determine whether a chunk will be visible using the current camera parameters. The horizon culling removes chunks that would not contribute to the final rendering when they are in the view frustum, but below the horizon - meaning that they are occluded by other chunks closer to the camera. Figure 3.37 shows this culling on a rendering on Mars.

Even through the Dynamic Scene Graph method as described above would enable the rendering of high-resolution surface terrains, it would require the dynamic generation of nodes in the scene graph tree, which would be prohibitive to realtime rendering. Using only a single scene graph node for the Earth, however, is insufficient as the Earth's radius is $6371 \mathrm{~km}$, which would lead to floating point precision errors below $6.371 \cdot 10^{7} \cdot 2^{-24} \approx 3.8$ meters. As there are data products with much higher resolution than this, a straightforward rendering of chunks would thus result in floating point jittering artifacts. In order to deal with this, the rendering system only renders chunks in model space until a certain subdivision level is reached. In the model space rendering, the vertices of the chunk's geometry are projected from the georeferenced map projection into the Cartesian model space of the globe, resulting in a curved surface model. Below the cutoff level, the chunks are rendered in camera space, in which only the corners of the chunk are projected using 64 bit floating point precision and then linearly interpolated on the GPU. Using 64 bit floating point precision for the entire rendering pipeline would be detrimental for the performance. The interpolation leads to flat chunks, but as this rendering method is only employed when close to the surface, the missing curvature of the globe is not noticeable. The cutoff level that determines which rendering method is used depends on the radius of the globe that is being rendered.

\subsubsection{Spacecraft Missions and Dissemination}

Spacecraft operations in the solar system are another astronomical scenario that benefits from using visualization in its accurate spatiotemporal context and the ability for dissemination to the general public. Paper I provides a high-level overview of OpenSpace with a focus on spacecraft missions and their usage as showcases for public dissemination. That paper is based on previous poster presentations at IEEE Vis [15] and the American Geophysical Union [17].

Scientific Problem The motivation to visualize spacecraft missions in detail stems directly from the complexity in their their design and engineering process. Particularly with missions that do not occur in the vicinity of Earth, there is no possibility to directly control a spacecraft's action in a timely fashion due to limitations in the speed of light. Instead, all movements and operations, especially if it is a flyby mission, have to be preprogrammed by the spacecraft controllers and are executed by the spacecraft remotely. These operations are carefully designed years in advance, at a time in which public interest in the particular mission is 


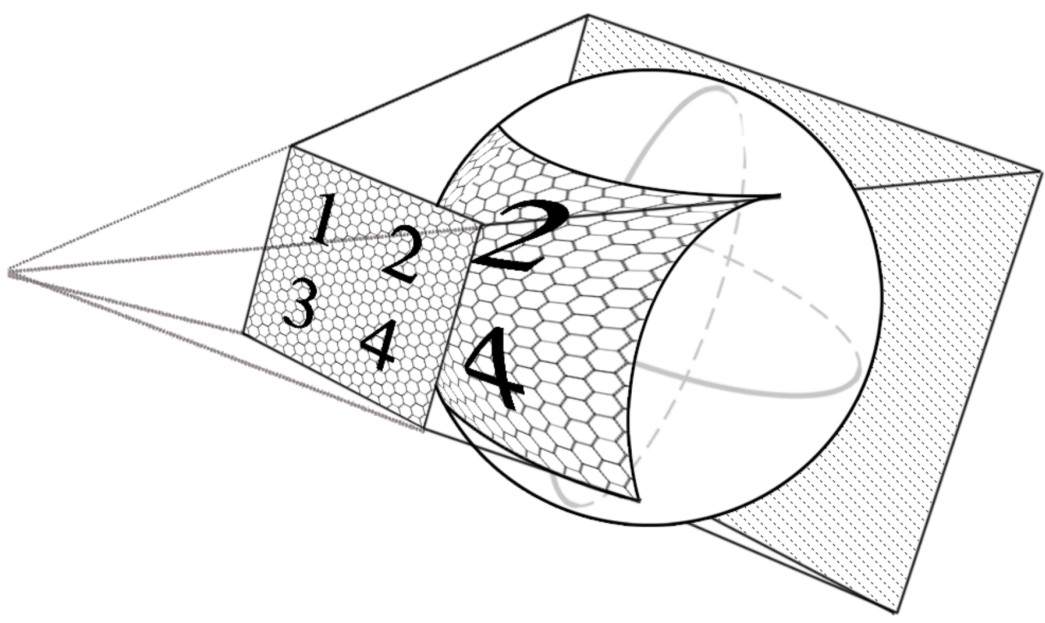

Figure 3.38: Showing a single image projected onto the curved surface of a globe. The image is projected onto the surface of the planet and is used to update a global image layer that covers the entire globe. Image copyright Michal Marcinkowski.

minimal. When the main goal of a mission is approaching, the public interest peaks, but the challenges faced by the engineers are rarely revealed when the final results of the spacecraft mission are being discussed in the media. Visualization can provide a valuable tool to explain to the general public how the final results of a mission were acquired, but at the same time use the audiences attention to highlight the complexity of the engineering that was necessary to arrive at the final results. A deeper insight into the spacecraft's operation requires an accurate placement of the spacecraft relative to their object of study and the visualization of instrument activity. Many of the interplanetary spacecraft contain imagers sensitive to the visible part of the electromagnetic spectrum. Displaying these images in their correct context can benefit an audience as it shows how a distant place looks like if a human observer were present instead of the spacecraft. Showing the process of image acquisition, on the other hand, provides an intuition into the planning of the spacecraft's actions ahead of time. Many of the planetary global images that are presented to the public are the result of, mostly manual, calibration of mosaicked images taken by the spacecraft and do not adequately accurately portray imaging conditions or the required post-production.

\section{Image Projections}

In order to better explain to the general public how the spacecraft's image acquisition operates, a rendering system was developed in collaboration with the New Horizons 


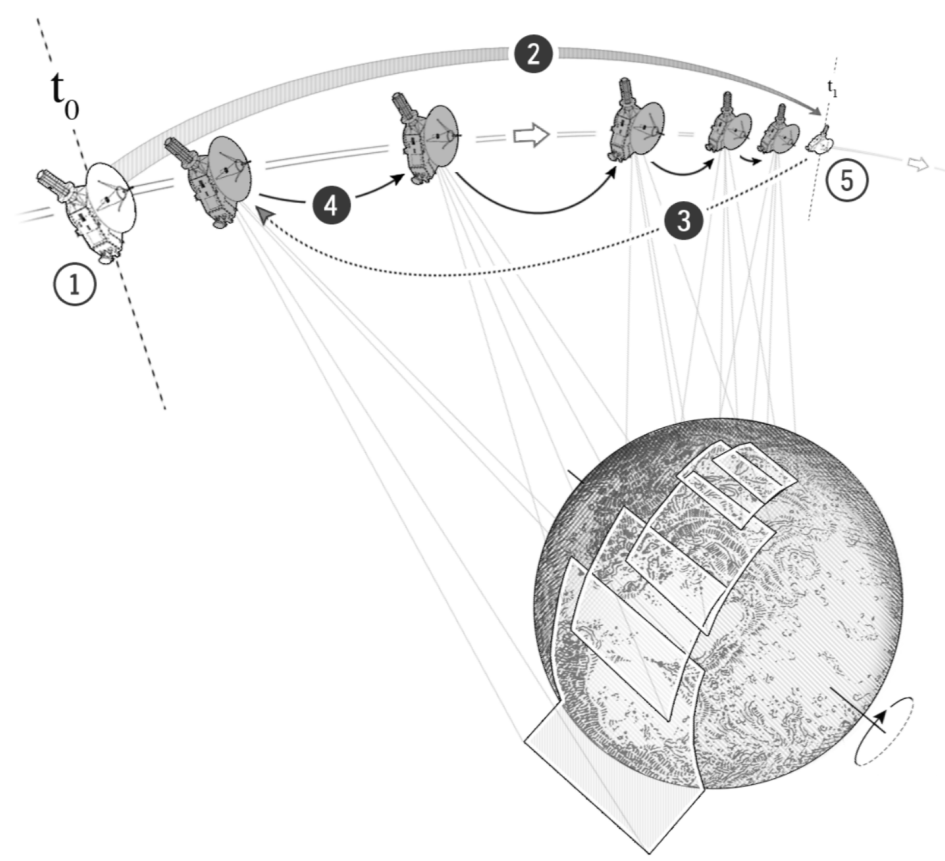

Figure 3.39: Using multiple projections during a flyby provides images of varying spatial resolution on the surface. These are all combined into the same global image layer and provide the user with an intuitive feedback why flyby missions usually provide global images with drastically varying spatial resolutions. Image copyright Michal Marcinkowski.

science team that utilizes the accurate position and orientation of a spacecraft to project the instrument's images onto a target body (see Figure 3.38). This method uses a projection method presented by Segal [105] and revisited by Everitt [39] that computes texture coordinates by using the spacecraft's location and orientation to compute a view matrix and the instrument parameters to compute a projection matrix for the algorithm and thus treats the imaging instrument as a slide projector. Given the acquisition time for each image, it is then possible to retain previously acquired images on the surface of the object in order to interactively show the construction of a full mosaic and better explain why, for example, there is a difference in spatial resolution in different areas of the object (see Figure 3.39).

This method consists of two components; 1 . the visualization of the instruments field-of-view, which especially for non-imaging instruments is not always rectangular; 2. the image projection onto the object itself. For the first component, the current instrument's boresight pointing vector in a global coordinate system is required 


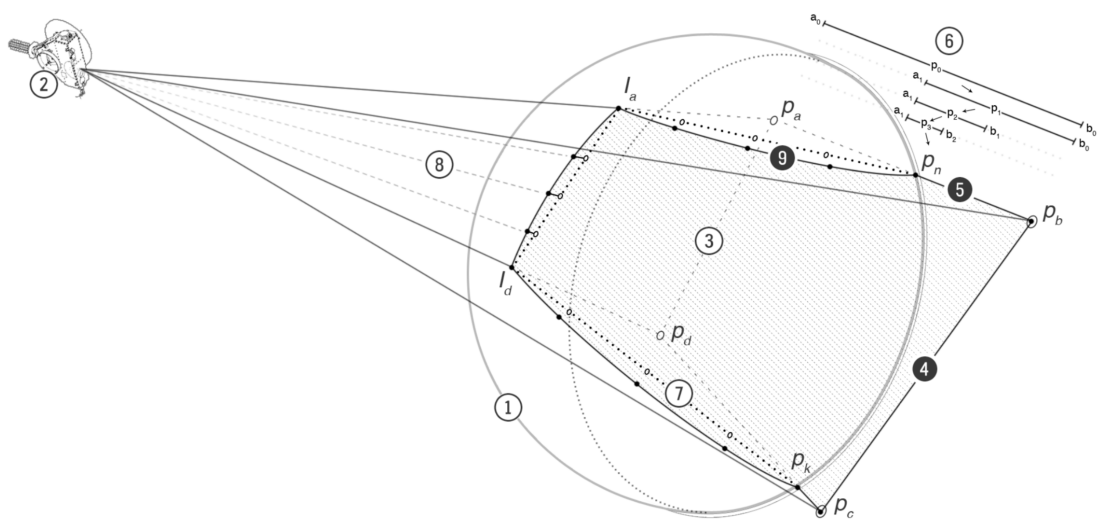

Figure 3.40: The instrument's field-of-view is rendered as a polygon that is modified to follow the surface of the object of study. Image copyright Michal Marcinkowski.

alongside its horizontal and vertical field-of-view parameters. This information is available through use of the SPICE library [2] which requires science-level accurate datasets. Fortunately, these are already created by the mission scientists and engineers in their regular workflow for a number of mission profiles in order to compare the expected image results for these different profiles. SPICE provides the boresight direction in the spacecraft's inertial reference frame, which can then be converted into the desired global reference frame, for example J2000. The SPICE datasets also include information about the field-of-view settings for rectangular imagers, which can be used immediately. For non-rectangular instruments, the vertex positions of the boundary polygon are returned instead. The vertices of the field-of-view representations are then radially extended from the spacecraft to the center of the observed object. If the field-of-view polygon intersects the geometry of the object of study, the vertices are offset to follow the curvature of the object. Figure 3.40 shows the reprojection of the field-of-view vertices $P_{a}$ and $P_{d}$ into $I_{a}$ and $I_{d}$ by using the known radius of the object (if the object is a planet), or by casting a probe ray (if the object is a complex shape). All intermediate points are then similarly reprojected in order to provide smooth transitions.

For the image projections, a list of all planned or performed image acquisitions is generated. In the case of future missions, such as OSIRIS-REx at the time of writing, this information is taken from a mission playbook that is generated by the scientists and engineers of the mission. The playbook includes, among others, information about the start and end times of the image acquisition as well as the object that is the target of the image. For historical missions, for example Rosetta, a hybrid approach is utilized that combines information from the playbook with metadata information included with the acquired and published images. If an 
image for a particular acquisition is already available, it is used in the projection step, otherwise a placeholder image is displayed instead. The image projections are rendered to a separate layer in order to provide the ability to cross-fade projections to show a comparison with an underlying global, preprocessed image.

When an image projection is requested, the projection layer texture of the object is attached to a framebuffer and the object is rendered using the view-projection matrix of the instrument. The currently projected image, placeholder or real image, is then blitted into the texture where the normalized device coordinates are between 0 and 1 , that is, where the object is inside the field-of-view of the instrument. This replaces the previous contents of the texture in these locations in order to show a progressive improvement in spatial resolution for missions where this is desireable.

Figure 3.41 shows this approach on an image mosaic of New Horizons on Pluto during the flyby on July $14^{\text {th }}, 2015$. The complete mosaic consists of 16 separate images that were taken in quick succession. A public event called "Breakfast at Pluto" using this system held on the day of closest approach that included 11 locations on all 5 continents and live streaming in which the mission scientists described the actions of the spacecraft live as they were unfolding.

Figures 3.42 and 3.43 show two missions, the Rosetta and OSIRIS-REx respectively, that either have orbited or will orbit around an irregular body. Rosetta's mission has ended in September 2016 with all images being released, whereas OSIRIS-REx' mission will end in September 2023 with its return to Earth. 


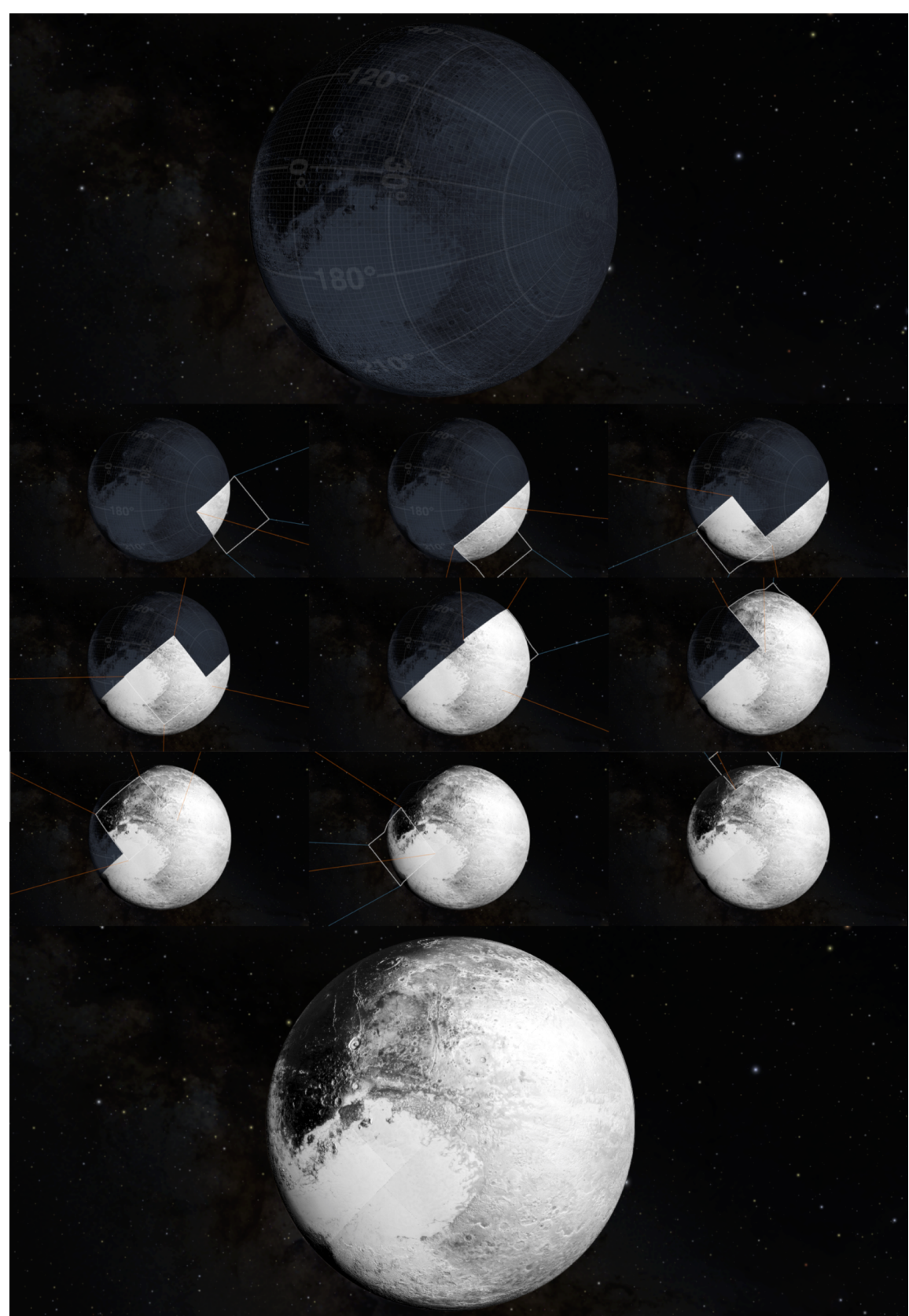

Figure 3.41: Projecting individual images of the New Horizons flyby show the composition of a complicated mosaicked image that is released to the public in its entirety. Showing individual images shows the engineering effort required to create the final image. Image copyright Michal Marcinkowski. 


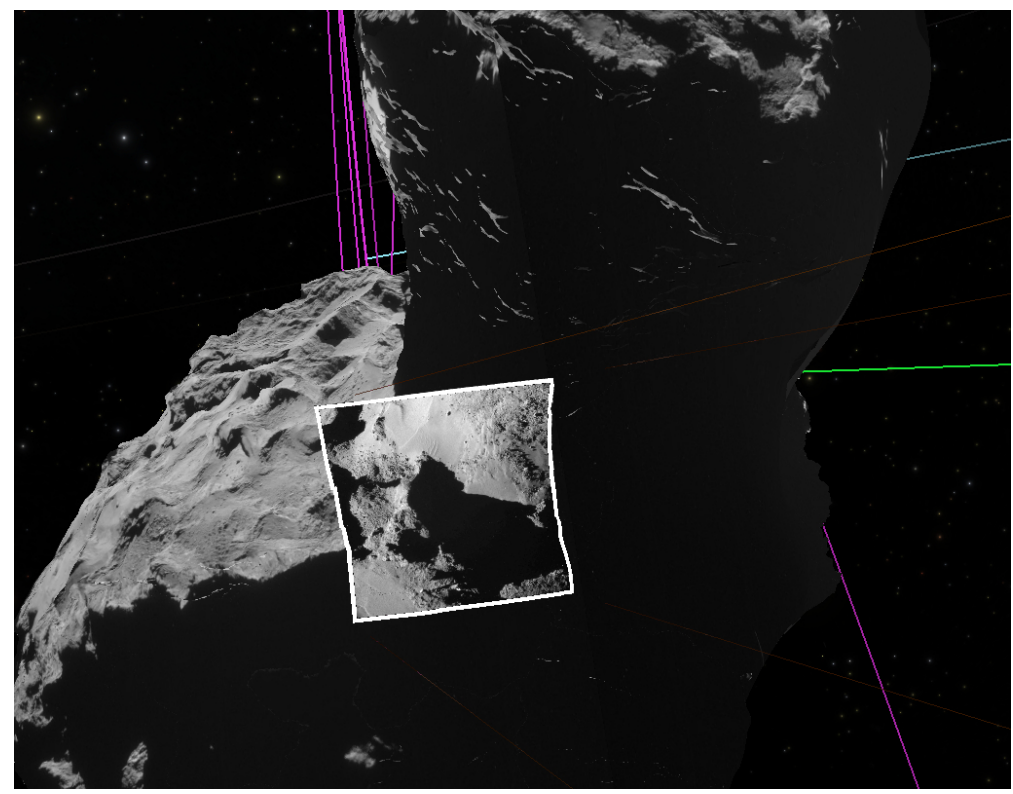

Figure 3.42: The Rosetta orbiter was in orbit around 67P/Churyumov-Gerasimenko for 2 years and acquired tens of thousands of images. The small image has just been taken during a closeup, showing the difference in resolution to a comet-wide image in the background taken earlier.

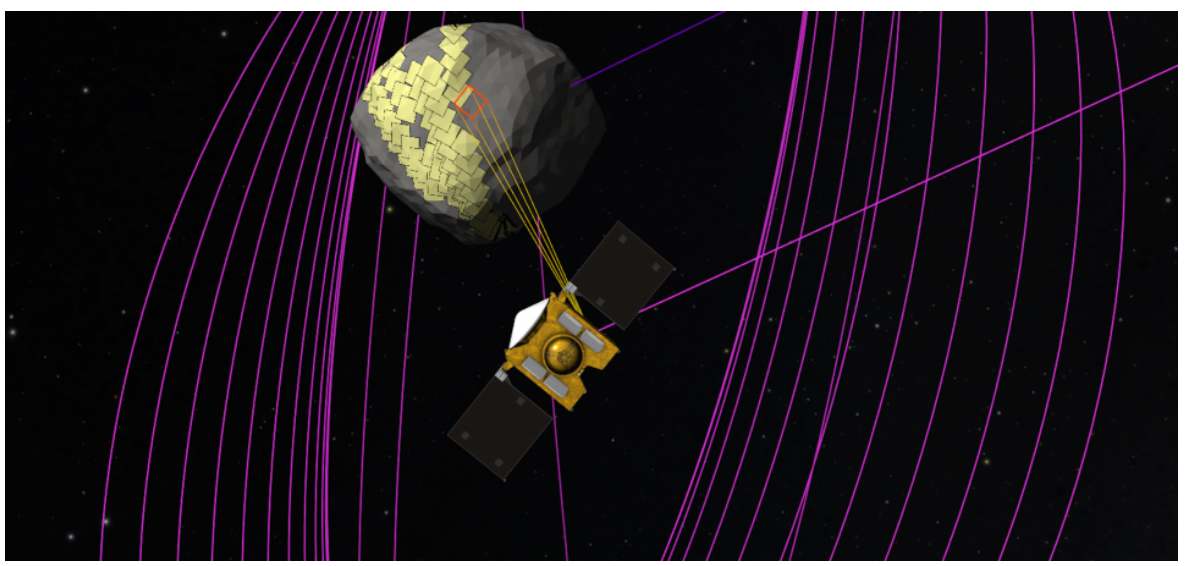

Figure 3.43: The OSIRIS-REx mission will arrive at the asteroid Bennu in August 2018 and depart in 2021 with a sample that is returned to Earth. As no images are yet available from this image campaign to find the touch-down location, placeholder images are projected instead. 



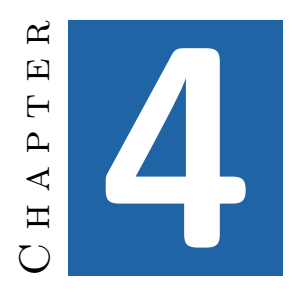

\section{Reflections}

The first part of this thesis presented the background and five examples for the design of visualization applications that utilize a combination of scientific visualization, information visualization, and visual analytics techniques in the context of the categories of visualization applications, Exploration, Analysis, and Communication.

This final chapter provides a summary of this first part of the thesis, includes personal reflections, and considers possible avenues for future work. The future work includes interesting research for myself, but also contains humble suggestions for the visualization community as a whole towards steps that might help to continue the accelerating growth of the field. In-depth discussions for concrete potential future work in each of the domains discussed in the previous chapter can be found in the publications attached in the second part of this thesis.

This thesis follows the line of argumentation that emphasizes the importance of considering the intended target audience when designing and creating visualization applications. This builds on previous research highlighting the difference between applications designed for explorational and presentational use cases by van Wijk [119] and the distinction into three (different) categories by Keim et al. [49] and Schulz et al. [104]. The papers included in this thesis illustrate that the explorational use cases presented by van Wijk can be further subdivided into initial exploratory hypothesis generation and repeated analyses. This leads to the three categories of visualization applications, Exploration, Analysis, and Communication described in Chapter 2. Table 2.1 showed a comparison of the definitions provided by Keim et al. [49], Schulz et al. [104], Brehmer et al. [22], van Wijk, where they overlap and how they differ. A common element between all classifications is the distinction between visualization applications that are designed for exploration and 
applications designed for presentation/communication. However, I would argue that there is a need for a more holistic approach for application design.

An essential component of these classifications are the task-based taxonomies and typologies presented by, among others, Shneiderman, Brehmner et al., Schulz et al., and others, some of which take the goal of the visualization tasks and thus the visualization application directly into account. However, are multiple deficiencies with this approach. In many taxonomies, while the differentiation between individual tasks is not always clear, the border between different user groups is much less pronounced. At most, such as in Miksch et al.'s Data-Users-Tasks Design Triangle [79], the users take second place and are differentiated by application domain, physical environment, social factors, or technical specifics. In my opinion, the existing successful taxonomies have to be adapted to take many more variables about the user groups that are impacted by visualization application and task design, such as domain knowledge, the level of integration, and the level of interactivity into account. This consideration would ask for a higher granularity of Communication-based visualization applications, which Brehmner et al. argue for.

Another aspect of the holistic approach to application design is signified by the quote by Kosara and Mackinlay "Visualization researchers often tacitly assume that the tools used for analysis are usable for presentation just as well as for their original purpose. We believe that to be a very limiting assumption, however." [53]. I would instead argue to the contrary that this is a very freeing assumption instead, due to the ongoing convergence of visualization applications aimed at Exploration and Communication. While there has always been exchanges between visualization applications designed for exploration and analysis, in the past the influence of these two categories on the communication aspect of visualization was less pronounced. The visualization application presented in Section 3.5.1 shows the potential of utilizing guided exploration for the public dissemination of complex scientific topics and how simplified exploratory methods can be used for the education of the general public and, at the same time, provide highly complex tools for domain scientists in the same framework. This combination allows scientists to rapidly disseminate their research finding without the active process of converting data into a new format for a different real-time application in order to use their findings for a guided, interactive presentation to the general public. This convergence of use cases is currently ongoing and induced by the availability of high performance GPUs on commodity hardware that enable the use of real, large scale and complex scientific data in both exploratory scenarios and public communication. As an additional benefit, the trend on focussing on the accessibility of visualization methods for the general public was present since the beginning of the visualization field, but has been increasing in the past years. The availability of visualization tools is especially beneficial for an interested general public. Providing access to sophisticated tools for the general public can provide a huge benefit to citizen science projects which increases the combined human knowledge and scientific engagement simultaneously. 
Naturally, this does not argue for the stagnation of fundamental visualization research but rather argues for an increase in the number of visualization researchers (another trend which can be observed at conferences).

Undoubtedly, the science of visualization and its tools have matured a lot in the past 25 years and now has a large influence on the scientific method in almost every field of research. High-impact scientific publications without accurate and visually impressive accompanying visualizations of their findings are unthinkable today. Furthermore, in many cases, no visualization experts are necessary to create these final visualizations as the access to sophisticated visualization tools has increased dramatically. The increasing ease-of-use of existing visualization techniques has thus led to a proliferation of knowledge about visualization techniques. While this, in my opinion, is a worthwhile trajectory of visualization research as it enables access to these tools to an increasing number of people, it also critically important to increase the users' visualization literacy alongside the tools' ease-of-use and shift more and more visualization techniques out of the ivory tower and into the hands of the knowledgeable public.

In my opinion, one of the most interesting and important developments that are currently ongoing is the intersection of visualization and machine learning methods. One of the most basic concepts of visualization is to connect the best abilities of the human mind with the best abilities of computational resources. Combining the natural intelligence of a human expert with the artificial intelligence of deep learning methods can, in my opinion, be hugely beneficial for the field. While, there are interesting debates on the viability of artificial intelligence to replace human cognition, these are better left outside the scope of this work. But if/until that happens, visualization techniques can certainly benefit from the rapid development in deep learning and vice versa, as many of the fundamental techniques, such as providing data abstractions, are shared between the two fields. In the reference frame of visualization as the field of using the human intelligence where it performs best and computational resources where they are most efficient, the introduction of artificial intelligence merely shifts that boundary, rather than remove it completely. This is illustrated by the quote from Stefan Lindholm: "One trend is the ever increasing number of arrows used whenever a visualization pipeline is illustrated. The narrow concept of a linear pipeline with a few fixed stages is going extinct" [61]. A suitable addition to this quote is the realization that it is important to remember that in the visualization pipeline, all arrows start and end at the human-in-the-loop. 



\section{Bibliography}

[1] C. Abraham, P. Cornillion, E. Matzner-Løber, and N. Molinari. Unsupervised Curve Clustering using B-Splines. Scandinavian Journal of Statistics, 30(3): 581-595, 2003. [page 27]

[2] C. H. Acton Jr. Ancillary Data Services of NASA's Navigation and Ancillary Information Facility. Planetary and Space Science, 44(1):65-70, 1996. [pages 52 and 68]

[3] E. Axelsson, J. Costa, C. T. Silva, C. Emmart, A. Bock, and A. Ynnerman. Dynamic Scene Graph: Enabling Scaling, Positioning, and Navigation in the Universe. Computer Graphics Forum, 36(3):459-468, 2017.

[4] J.-P. Balabanian, I. Viola, E. Ona, R. Patel, and E. Gröller. Sonar Explorer: A New Tool for Visualization of Fish Schools from 3D Sonar Data. In Eurographics/IEEE-VGTC Symposium on Visualization, pages 155-162, 2007. [pages 12 and 53]

[5] K.-J. Bathe and E. L. Wilson. Numerical Methods in Finite Element Analysis. Prentice-Hall, 1976. [page 24]

[6] A. L. Benabid, S. Chabardes, J. Mitrofanis, and P. Pollak. Deep Brain Stimulation of the Subthalamic Nucleus for the Treatment of Parkinson's Disease. Lancet Neurology, 8(1):67-81, 2009. [page 31]

[7] A. Benazzouz, S. Breit, A. Koudsie, P. Pollak, P. Krack, and A.-L. Benabid. Intraoperative Microrecordings of the Subthalamic Nucleus in Parkinson's Disease. Movement disorders, 17(3):145-149, 2002. [page 31]

[8] J. Beyer, M. Hadwiger, S. Wolfsberger, and K. Bühler. High-Quality Multimodal Volume Rendering for Preoperative Planning of Neurosurgical Interventions. IEEE Transactions on Visualization and Computer Graphics, 13 (6):1696-1703, 2007. [page 33]

[9] K. Bladin, E. Axelsson, E. Broberg, C. Emmart, P. Ljung, A. Bock, and A. Ynnerman. Globe Browsing: Contextualized Spatio-Temporal Planetary Surface Visualization. IEEE Transactions on Visualization and Computer Graphics, 24(1):802-811, 2017. 
[10] A. Bock, E. Sundén, B. Liu, B. Wuensche, and T. Ropinski. Coherency-Based Curve Compression for High-Order Finite Element Model Visualization. IEEE Transactions on Visualization and Computer Graphics, 18(12):2315-2324, 2012.

[11] A. Bock, N. Lang, G. Evangelista, R. Lehrke, and T. Ropinski. Guiding Deep Brain Stimulation Interventions by Fusing Multimodal Uncertainty Regions. In Proceedings of the Pacific Visualization Symposium (PacificVis), pages 97-104. IEEE, 2013.

[12] A. Bock, A. Kleiner, J. Lundberg, and T. Ropinski. An Interactive Visualization System for Urban Search \& Rescue Mission Planning. In International Symposium on Safety, Security, and Rescue Robotics. IEEE, 2014.

[13] A. Bock, A. Kleiner, J. Lundberg, and T. Ropinski. Supporting Urban Search \& Rescue Mission Planning through Visualization-Based Analysis. In Vision, Modeling $\&$ Visualization. The Eurographics Association, 2014.

[14] A. Bock, M. L. Mays, L. Rastaetter, A. Ynnerman, and T. Ropinski. VCMass: A Framework for Verification of Coronal Mass Ejection Ensemble Simulations. In Proceedings of the Scientific Visualization Conference (Poster). IEEE, 2014. [page 47]

[15] A. Bock, M. Marcinkowski, J. Kilby, C. Emmart, and A. Ynnerman. OpenSpace: Public Dissemination of Space Mission Profiles. In Proceedings of the Scientific Visualization Conference (Poster), pages 141-142. IEEE, 2015. [page 65]

[16] A. Bock, A. Pembroke, M. L. Mays, L. Rastaetter, A. Ynnerman, and T. Ropinski. Visual Verification of Space Weather Ensemble Simulations. In Proceedings of the Scientific Visualization Conference. IEEE, 2015.

[17] A. Bock, A. Pembroke, M. L. Mays, and A. Ynnerman. OpenSpace: An Open-Source Framework for Data Visualization and Contextualization. In American Geophysical Union Fall Meeting Abstracts, pages IN42A-03, 2015. [page 65]

[18] A. Bock, Å. Svensson, A. Kleiner, J. Lundberg, and T. Ropinski. A Visualization-Based Analysis System for Urban Search \& Rescue Mission Planning Support. Computer Graphics Forum, 36(6):148-159, 2016.

[19] A. Bock, E. Axelsson, K. Bladin, J. Costa, G. Payne, M. Territo, J. Kilby, E. Myers, M. M. Kuznetsova, C. Emmart, and A. Ynnerman. OpenSpace: An Open-Source Astrovisualization Framework. Journal of Open-Source Software, 2(15):281, 2017. [page 56] 
[20] A. Bock, C. Emmart, M. Kuznetsova, and A. Ynnerman. OpenSpace: Changing the Narrative of Public Disseminations in Astronomical Visualization from What to How. IEEE Computer Graphics and Applications, Special Issue - Applied Vis, 38(3), 2018, to appear May/June 2018.

[21] D. Borland and R. M. Taylor II. Rainbow Color Map (Still) Considered Harmful. IEEE Computer Graphics and Applications, 27(2):14-17, 2007. [page 9]

[22] M. Brehmer and T. Munzner. A Multi-Level Typology of Abstract Visualization Tasks. IEEE Transactions on Visualization and Computer Graphics, 19 (12):2376-2385, 2013. [pages 18, 19, 20, and 73]

[23] M. Brehmer, M. Sedlmair, S. Ingram, and T. Munzner. Visualizing Dimensionally-Reduced Data: Interviews with Analysts and a Characterization of Task Sequences. In Proceedings of the Workshop on Beyond Time and Errors: Novel Evaluation Methods for Visualization. ACM, 2014. [page 16]

[24] C. R. Butson, S. E. Cooper, J. M. Henderson, and C. C. McIntyre. PatientSpecific Analysis of the Volume of Tissue Activated during Deep Brain Stimulation. Neuroimage, 34(2):661-670, 2007. [page 31]

[25] S. K. Card, J. D. Mackinlay, and B. Shneiderman. Readings in Information Visualization: Using Vision to Think. Morgan Kaufmann, 1999. [pages 4 and 5]

[26] E. Catmull and R. Rom. A Class of Local Interpolating Splines. Computer Aided Geometric Design, 74:317-326, 1974. [pages 25 and 44]

[27] R. C. Colaninno and A. Vourlidas. Analysis of the Velocity Field of CMEs using Optical Flow Methods. The Astrophysical Journal, 652(2):1747, 2006. [page 51]

[28] P. Cozzi and K. Ring. 3D Engine Design for Virtual Globes. CRC Press, 2011. [page 63]

[29] A. Cuyt, B. Verdonk, S. Becuwe, and P. Kuterna. A Remarkable Example of Catastrophic Cancellation Unraveled. Computing, 66(3):309-320, 2001. [page 58]

[30] R. Damadian. Tumor Detection by Nuclear Magnetic Resonance. Science, 171(3976):1151-1153, 1971. [page 13]

[31] J. Danskin and P. Hanrahan. Fast Algorithms for Volume Ray Tracing. In Proceedings of the 1992 workshop on volume visualization, pages 91-98. ACM, 1992. [page 15] 
[32] J. de La Beaujardiere. OpenGIS Web Map Server Implementation Specification. Implementation standard, Open Geospatial Consortium Inc, 2006. [page 62]

[33] T. DeFanti, M. Brown, and B. McCormick. Visualization: Expanding Scientific and Engineering Research Opportunities. IEEE Computer, 22(8):12-16, 1989. [page 4]

[34] M. E. Dieckmann, A. Bock, H. Ahmed, D. Doria, G. Sarri, A. Ynnerman, and M. Borghesi. Shocks in Unmagnetized Plasma with a Shear Flow: Stability and Magnetic Field Generation. Journal of Plasma Physics, 22(7):072104, 2015 .

[35] S. Dos Santos and K. Brodlie. Gaining Understanding of Multivariate and Multidimensional Data Through Visualization. Computers \& Graphics, 28 (3):311-325, 2004. [page 9]

[36] R. A. Drebin, L. Carpenter, and P. Hanrahan. Volume Rendering. ACM Computer Graphics, 22(4):65-74, 1988. [page 14]

[37] K. A. Ericsson and H. A. Simon. Verbal Reports as Data. Psychological Review, 87(3):215, 1980. [page 47]

[38] C. Everitt. Interactive Order-Independent Transparency. White paper, Nvidia, 2001. [page 28]

[39] C. Everitt. Projective texture mapping. White paper, Nvidia, 2001. [pages 43 and 67$]$

[40] N. Ferreira, J. Poco, H. T. Vo, J. Freire, and C. T. Silva. Visual Exploration of Big Spatio-Temporal Urban Data: A Study of New York City Taxi Trips. IEEE Transactions on Visualization and Computer Graphics, 19(12):21492158, 2013. [page 21]

[41] G. Galilei. Sidereus Nuncius. Thomas Baglioni, 1610. [page 56]

[42] B. Guenter and R. Parent. Computing the Arc Length of Parametric Curves. IEEE Computer Graphics and Applications, 10(3):72-78, 1990. [page 29]

[43] R. B. Haber and D. A. McNabb. Visualization Idioms: A Conceptual Model for Scientific Visualization Systems. In Visualization in Scientific Computing, pages 74-93. IEEE, 1990. [page 9]

[44] G.-M. Hariz, M. Lindberg, and A. T. Bergenheim. Impact of Thalamic Deep Brain Stimulation on Disability and Health-Related Quality of Life in Patients with Essential Tremor. Journal of Neurology, Neurosurgery and Psychiatry, 72:47-52, 2002. [page 31] 
[45] P. E. Hart, N. J. Nilsson, and B. Raphael. A Formal Basis for the Heuristic Determination of Minimum Cost Paths. Transactions on Systems Science and Cybernetics, 4(2):100-107, 1968. [page 41]

[46] J. A. Hartigan. Clustering Algorithms, volume 209. Wiley, 1975. [page 27]

[47] G. N. Hounsfield. Computed Medical Imaging. Science, 210(4465):22-28, 1980. [page 13]

[48] D. Jönsson, M. Falk, and A. Ynnerman. Intuitive Exploration of Volumetric Data using Dynamic Galleries. IEEE Transactions on Visualization and Computer Graphics, 22(1):896-905, 2016. [page 22]

[49] D. A. Keim, F. Mansmann, J. Schneidewind, and H. Ziegler. Challenges in Visual Data Analysis. In Conference on Information Visualization, pages 9-16. IEEE, 2006. [pages 20 and 73]

[50] R. M. Kirby and M. Meyer. Visualization Collaborations: What Works and Why. IEEE Computer Graphics and Applications, 33(6):82-88, 2013. [pages 4 and 16]

[51] S. Knowles, J. Picone, S. Thonnard, and A. Nicholas. The Effect of Atmospheric Drag on Satellite Orbits during the Bastille Day Event. Solar Physics, 204(1):387-397, 2001. [page 47]

[52] A. H. König, H. Doleisch, and E. Gröller. Multiple Views and Magic MirrorsfMRI Visualization of the Human Brain. Technical Report TR-186-2-9908, Institute of Computer Graphics and Algorithms, Vienna University of Technology, 1999. [page 11]

[53] R. Kosara and J. Mackinlay. Storytelling: The Next Step for Visualization. IEEE Computer, 46(5):44-50, 2013. [pages 21 and 74]

[54] J. Krüger and R. Westermann. Acceleration Techniques for GPU-Based Volume Rendering. In Visualization. IEEE, 2003. [pages 15 and 28]

[55] A. Kumpf, B. Tost, M. Baumgart, M. Riemer, R. Westermann, and M. Rautenhaus. Visualizing Confidence in Cluster-based Ensemble Weather Forecast Analyses. IEEE Transactions on Visualization and Computer Graphics, 24 (1):109-119, 2018. [page 21]

[56] C. Larman and V. R. Basili. Iterative and Incremental Development: A Brief History. IEEE Computer, 36(6):47-56, 2003. [page 16]

[57] F. Lenz, J. Dostrvsky, H. Kwan, et al. Methods for Microstimulation and Recording of Single Neurons and Evoked Potentials in the Human Central Nervous System. Journal of Neurosurgery, 68(4):630-634, 1988. [page 31] 
[58] M. Levoy. Display of Surfaces from Volume Data. IEEE Computer Graphics and Applications, 8(3):29-37, 1988. [page 14]

[59] C. Lewis and J. Rieman. Task-Centered User Interface Design: A Practical Introduction. University of Colorado, Boulder, 1993. [page 47]

[60] R. Likert. A Technique for the Measurement of Attitudes. Archives of Psychology, 140:5-55, 1932. [page 36]

[61] S. Lindholm. Medical Volume Visualization Beyond Single Voxel Values. PhD thesis, Linköping University, 2014. [page 75]

[62] S. Lindholm and A. Bock. Poor Man's Rendering of Segmented Data. In Proceedings of SIGRAD, volume 94, pages 49-54, 2013.

[63] S. Lindholm, M. Falk, E. Sundén, A. Bock, A. Ynnerman, and T. Ropinski. Hybrid Data Visualization Based On Depth Complexity Histogram Analysis. Computer Graphics Forum, 34(1):74-85, 2014. [page 54]

[64] B. Liu, A. Bock, T. Ropinski, M. Nash, P. Nielsen, and B. Wuensche. GPUAccelerated Direct Volume Rendering of Finite Element Data Sets. In Proceedings of the Conference on Image and Vision Computing New Zealand, pages 109-114. ACM, 2012. [page 25]

[65] Y. Liu and G. Nejat. Robotic Urban Search and Rescue: A Survey from the Control Perspective. Journal of Intelligent $\&$ Robotic Systems, 72(2):147-165, 2013. [page 37]

[66] P. Ljung. Adaptive Sampling in Single Pass, GPU-based Raycasting of multiresolution Volumes. In Volume Graphics. The Eurographics Association, 2006. [page 29]

[67] B. Lorensen. On the Death of Visualization. In Position Papers NIH/NSF Proceedings of the Fall Workshop Visualization Research Challenges, volume 1, 2004. [pages 6 and 7]

[68] T. Luft, C. Colditz, and O. Deussen. Image Enhancement by Unsharp Masking the Depth Buffer. ACM Transactions on Graphics, 25(3):1206-1213, 2006. [pages 33 and 43]

[69] K.-L. Ma, I. Liao, J. Frazier, H. Hauser, and H.-N. Kostis. Scientific Storytelling using Visualization. IEEE Computer Graphics and Applications, 32 (1):12-19, 2012. [page 61]

[70] G. Marchionini. Exploratory Search: From Finding to Understanding. Communications of the ACM, 49(4):41-46, 2006. [page 20] 
[71] S. Martelli, L. Nofrini, P. Vendruscolo, and A. Visani. Criteria of Interface Evaluation for Computer Assisted Surgery Systems. International Journal of Medical Informatics, 72(1):35-45, 2003. [page 36]

[72] J. Maso, K. Pomakis, and N. Julia. OpenGIS Web Map Tile Service Implementation Standard. Implementation standard, Open Geospatial Consortium, 2010. [page 62]

[73] N. Max. Optical Models for Direct Volume Rendering. IEEE Transactions on Visualization and Computer Graphics, 1(2):99-108, 1995. [page 14]

[74] N. Max and M. Chen. Local and Global Illumination in the Volume Rendering Integral. In Dagstuhl Follow-Ups, volume 1. Schloss Dagstuhl-LeibnizZentrum für Informatik, 2010. [page 14]

[75] T. Maynard, N. Smith, and S. Gonzalez. Solar Storm Risk to the North American Electric Grid. Risk reports, Lloyd's, 2013. [page 48]

[76] M. Mays, A. Taktakishvili, A. Pulkkinen, P. MacNeice, L. Rastätter, D. Odstrcil, L. Jian, I. Richardson, J. LaSota, Y. Zheng, et al. Ensemble Modeling of CMEs Using the WSA-ENLIL + Cone Model. Solar Physics, 290(6): 1775-1814, 2015. [page 49]

[77] A. S. McEwen, R. Heyd, S. Sutton, Y. Espinosa, A. Fennema, R. Leis, G. McArthur, C. Schaller, M. Chojnacki, L. Keszthelyi, K. Becker, R. Kirk, and E. Eliason. For the People: HiRISE Data Products. In Lunar and Planetary Science Conference, volume 47, page 1371, 2016. [page 63]

[78] M. Meyer, M. Sedlmair, P. S. Quinan, and T. Munzner. The Nested Blocks and Guidelines Model. Information Visualization, 14(3):234-249, 2015. [page 18]

[79] S. Miksch and W. Aigner. A Matter of Time: Applying a Data-Users-Tasks Design Triangle to Visual Analytics of Time-Oriented Data. Computers 8 Graphics, 38:286-290, 2014. [page 74]

[80] S. Miočinović, J. Zhang, W. Xu, et al. Stereotactic Neurosurgical Planning, Recording, and Visualization for Deep Brain Stimulation in Non-Human Primates. Journal of Neuroscience Methods, 162(1-2):32-41, 2007. [page 33]

[81] K. Moreland. A Survey of Visualization Pipelines. IEEE Transactions on Visualization and Computer Graphics, 19(3):367-378, 2013. [page 9]

[82] P. Morrison and P. Morrison. Powers of Ten: A Book about the Relative Size of Things in the Universe and the Effect of Adding Another Zero. Scientific American, 1982. [page 57] 
[83] J. D. Mulder, J. J. van Wijk, and R. van Liere. A Survey of Computational Steering Environments. Future Generation Computer Systems, 15(1):119-129, 1999. [page 10]

[84] T. Munzner. A Nested Model for Visualization Design and Validation. IEEE Transactions on Visualization and Computer Graphics, 15(6):921-928, 2009. [page 17]

[85] T. Munzner. Visualization Analysis and Design. CRC Press, 2014. [pages 5 and 15]

[86] K. T. Nguyen, A. Bock, A. Ynnerman, and T. Ropinski. Deriving and Visualizing Uncertainty in Kinetic PET Modeling. In Proceedings of the Workshop on Visual Computing for Biology and Medicine, pages 107-114. Eurographics, 2012.

[87] C. North. Toward Measuring Visualization Insight. IEEE Computer Graphics and Applications, 26(3):6-9, 2006. [page 4]

[88] C. North and B. Shneiderman. A Taxonomy of Multiple Window Coordination. Technical Report CS-TR-3854, Department of Computer Science, University of Maryland, 1997. [page 16]

[89] R. Pausch, D. Proffitt, and G. Williams. Quantifying Immersion in Virtual Reality. In Proceedings of the Conference on Computer Graphics and Interactive Techniques, pages 13-18. ACM, 1997. [page 45]

[90] B. T. Phong. Illumination for Computer Generated Pictures. Communications of the ACM, 18(6):311-317, 1975. [page 43]

[91] R. Pirjola, A. Viljanen, O. Amm, and A. Pulkkinen. Power and Pipelines (Ground Systems). In Proceedings of a Workshop on Space Weather, 1999. [page 48]

[92] B. Preim and C. P. Botha. Visual Computing for Medicine: Theory, Algorithms, and Applications. Newnes, 2013. [page 5]

[93] T.-M. Rhyne, M. Tory, T. Munzner, M. O. Ward, C. R. Johnson, and D. H. Laidlaw. Information and Scientific Visualization: Separate but Equal or Happy Together at Last. In Visualization, volume 3, pages 611-614. IEEE, 2003. [page 6]

[94] E. Richter, T. Hogue, W. Halliday, et al. Determining the Position and Size of the Subthalamic Nucleus based on Magnetic Resonance Imaging Results in Patients with Advanced Parkinson Disease. Journal of Neurosurgery, 100 (3):541-546, 2004. [page 31] 
[95] N. Ritter and M. Ruth. GeoTIFF Format Specification. Technical report, GeoTIFF Working Group, 2000. [page 63]

[96] N. Rönnberg and J. Jimmy. Interactive Sonification for Visual Dense Data Displays. In Proceedings of the Interactive Sonification Workshop, pages 63-67, 2016. [page 6]

[97] W. C. Röntgen. On a New Kind of Rays. Science, 3(59):227-231, 1896. [page 12]

[98] W. W. Royce. Managing the development of large software systems: concepts and techniques. In Proceedings of the Conference on Software Engineering, pages 328-338. IEEE, 1987. [page 17]

[99] A. Rungta, B. Summa, D. Demir, P.-T. Bremer, and V. Pascucci. ManyVis: Multiple Applications in an Integrated Visualization Environment. IEEE Transactions on Visualization and Computer Graphics, 19(12):2878-2885, 2013. [page 16]

[100] S. Russell and P. Norvig. Artificial Intelligence: A Modern Approach. Pearson, 1995. [page 41]

[101] R. B. Rusu and S. Cousins. 3D is Here: Point Cloud Library (PCL). In Conference on Robotics and Automation, pages 1-4. IEEE, 2011. [page 39]

[102] P. Sabella. A Rendering Algorithm for Visualizing 3D Scalar Fields. ACM Computer Graphics, 22(4):51-58, 1988. [page 14]

[103] C. Sabol, R. Burns, and S. Wallace. Analysis of the Telstar-401/GOES-10 Close Approach using the Raven Telescope. Advances in the Astronautical Sciences, 99(1):317-332, 1998. [page 47]

[104] H.-J. Schulz, T. Nocke, M. Heitzler, and H. Schumann. A Design Space of Visualization Tasks. IEEE Transactions on Visualization and Computer Graphics, 19(12):2366-2375, 2013. [pages 19, 20, 21, and 73]

[105] M. Segal, C. Korobkin, R. van Widenfelt, J. Foran, and P. Haeberli. Fast Shadows and Lighting Effects using Texture Mapping. In SIGGRAPH, 1992. [pages 43 and 67]

[106] C. E. Shannon. Communication in the Presence of Noise. Proceedings of the Institute of Radio Engineers, 37(1):10-21, 1949. [page 15]

[107] H.-W. Shen, L.-J. Chiang, and K.-L. Ma. A Fast Volume Rendering Algorithm for Time-Varying Fields Using a Time-Space Partitioning (TSP) Tree. In Proceedings of the Conference on Visualization, pages 371-377. IEEE, 1999. [page 12] 
[108] B. Shneiderman. The Eyes Have It: A Task by Data Type Taxonomy for Information Visualizations. In Symposium on Visual Languages, pages 336-343. IEEE, 1996. [pages 12 and 18]

[109] J. Snow. On the Mode of Communication of Cholera. John Churchill, 1855. [page 3]

[110] P. Starr, C. Christine, P. Theodosopoulos, N. Lindsey, D. Byrd, A. Mosley, and W. Marks Jr. Implantation of Deep Brain Stimulators into the Subthalamic Nucleus: Technical Approach and Magnetic Resonance Imaging-Verified Lead Locations. Journal of Neurosurgery, 97(2):370-87, 2002. [page 31]

[111] D. Sun, S. Roth, and M. J. Black. Secrets of Optical Flow Estimation and Their Principles. In Conference on Computer Vision and Pattern Recognition, pages 2432-2439. IEEE, 2010. [page 52]

[112] E. Sundén, A. Bock, D. Jönsson, A. Ynnerman, and T. Ropinski. Interaction Techniques as a Communication Channel when Presenting 3D Visualizations. In VIS International Workshop on 3DVis, pages 61-64. IEEE, 2014.

[113] M. Törnros. Interactive Visualization of Space Weather Data, 2013. [page 47]

[114] M. Tory. Mental Registration of 2D and 3D Visualizations (An Empirical Study). In Proceedings of Visualization. IEEE, 2003. [page 11]

[115] M. Tory and T. Möller. A Model-Based Visualization Taxonomy. Technical Report TR 2002-06, School of Computing Science, Simon Fraser University, 2002. [page 6]

[116] E. R. Tufte. Envisioning Information. Optometry ES Vision Science, 68(4): 322-324, 1991. [page 3]

[117] E. R. Tufte and P. Graves-Morris. The Visual Display of Quantitative Information. Graphics press, 1983. [page 3]

[118] T. Ulrich. Rendering Massive Terrains using Chunked Level of Detail Control. In Proceedings of SIGGRAPH. ACM, 2002. [page 63]

[119] J. J. Van Wijk. The Value of Visualization. In Visualization, pages 79-86. IEEE, 2005. [pages 7, 15, 19, 20, and 73]

[120] J. J. Van Wijk. Bridging the Gaps. IEEE Computer Graphics and Applications, 26(6):6-9, 2006. [page 16]

[121] J. J. Van Wijk. Views on visualization. IEEE Transactions on Visualization and Computer Graphics, 12(4):421-432, 2006. [page 6] 
[122] R. Vondrak, J. Keller, G. Chin, and J. Garvin. Lunar Reconnaissance Orbiter (LRO): Observations for Lunar Exploration and Science. Space Science Reviews, 150(1-4):7-22, 2010. [page 63]

[123] M. Q. Wang Baldonado, A. Woodruff, and A. Kuchinsky. Guidelines for Using Multiple Views in Information Visualization. In Proceedings of the Conference on Advanced Visual Interfaces, pages 110-119. ACM, 2000. [page 16]

[124] M. O. Ward, G. Grinstein, and D. Keim. Interactive Data Visualization: Foundations, Techniques, and Applications. CRC Press, 2010. [page 15]

[125] F. Warmerdam. The Geospatial Data Abstraction Library. In Open Source Approaches in Spatial Data Handling, pages 87-104. Springer, 2008. [page 63]

[126] D. Weiskopf, K.-L. Ma, J. J. van Wijk, R. Kosara, and H. Hauser. SciVis, InfoVis - Bridging the Community Divide. In Proceedings of the Visualization Conference. IEEE, 2006. [page 6]

[127] M. Wertheimer. Untersuchungen zur Lehre von der Gestalt. Psychologische Forschung, 1(1):47-58, 1922. [page 2]

[128] P. C. Wong and J. Thomas. Guest Editors' Introduction-Visual Analytics. IEEE Computer Graphics and Applications, 24(5):20-21, 2004. [page 6]

[129] B. Wünsche and A. A. Young. The Visualization and Measurement of Left Ventricular Deformation using Finite Element Models. Journal of Visual Languages and Computing, 14(4):299-326, 2003. [page 24]

[130] R. Yagel and Z. Shi. Accelerating Volume Animation by Space-Leaping. In Proceedings of the Conference on Visualization, pages 62-69, 1993. [page 15]

[131] A. A. Young and L. Axel. Three-Dimensional Motion and Deformation of the Heart Wall: Estimation with Spatial Modulation of Magnetization - A Model-Based Approach. Radiology, 185(1):241-247, 1992. [page 24]

[132] A. A. Young, D. L. Kraitchman, L. Dougherty, and L. Axel. Tracking and Finite Element Analysis of Stripe Deformation in Magnetic Resonance Tagging. IEEE Transactions on Medical Imaging, 14(3):413-421, 1995. [page 24]

[133] W. Zhao, D. Nister, and S. Hsu. Alignment of Continuous Video onto 3D Point Clouds. IEEE Transactions on Pattern Analysis and Machine Intelligence, 27(8):1305-1318, 2005. [page 44] 



\section{Papers}

The papers associated with this thesis have been removed for copyright reasons. For more details about these see:

$\underline{\text { http://urn.kb.se/resolve?urn=urn:nbn:se:liu:diva-147975 }}$ 